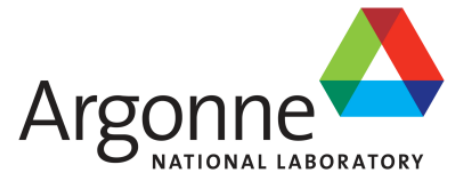

ANL/NE-16/50 Rev. 1.0

\title{
GAMSOR: Gamma Source Preparation and DIF3D Flux Solution
}

Revision 1.0

Nuclear Engineering Division 


\author{
About Argonne National Laboratory \\ Argonne is a U.S. Department of Energy laboratory managed by UChicago \\ Argonne, LLC under contract DE-AC02-06CH11357. The Laboratory's main facility \\ is outside Chicago, at 9700 South Cass Avenue, Argonne, Illinois 60439. For \\ information about Argonne \\ and its pioneering science and technology programs, see www.anl.gov.
}

\title{
DOCUMENT AVAILABILITY
}

Online Access: U.S. Department of Energy (DOE) reports produced after 1991 and a growing number of pre-1991 documents are available free via DOE's SciTech Connect (http://www.osti.gov/scitech/)

\author{
Reports not in digital format may be purchased by the public \\ from the National Technical Information Service (NTIS): \\ U.S. Department of Commerce \\ National Technical Information \\ Service 5301 Shawnee Rd \\ Alexandria, VA 22312 \\ www.ntis.gov \\ Phone: (800) 553-NTIS (6847) or (703) 605-6000 \\ Fax: (703) 605-6900 \\ Email: orders@ntis.gov
}

\author{
Reports not in digital format are available to DOE and DOE contractors \\ from the Office of Scientific and Technical Information (OSTI): \\ U.S. Department of Energy \\ Office of Scientific and Technical Information \\ P.O. Box 62 \\ Oak Ridge, TN 37831-0062 \\ www.osti.gov \\ Phone: (865) 576-8401 \\ Fax: (865) 576-5728 \\ Email: reports@osti.gov
}

\begin{abstract}
Disclaimer
This report was prepared as an account of work sponsored by an agency of the United States Government. Neither the United States Government nor any agency thereof, nor UChicago Argonne, LLC, nor any of their employees or officers, makes any warranty, express or implied, or assumes any legal liability or responsibility for the accuracy, completeness, or usefulness of any information, apparatus, product, or process disclosed, or represents that its use would not infringe privately owned rights. Reference herein to any specific commercial product, process, or service by trade name, trademark, manufacturer, or otherwise, does not necessarily constitute or imply its endorsement, recommendation, or favoring by the United States Government or any agency thereof. The views and opinions of document authors expressed herein do not necessarily state or reflect those of the United States Government or any agency thereof, Argonne National Laboratory, or UChicago Argonne, LLC.
\end{abstract}




\section{GAMSOR: Gamma Source Preparation and DIF3D Flux Solution}

\section{Revision 1.0}

prepared by

M. A. Smith, C. H. Lee, and R. N. Hill

Nuclear Engineering Division, Argonne National Laboratory

June 28, 2017 



\section{SUMMARY}

Nuclear reactors that rely upon the fission reaction have two modes of thermal energy deposition in the reactor system: neutron absorption and gamma absorption. The gamma rays are typically generated by neutron capture reactions or during the fission process which means the primary driver of energy production is of course the neutron interaction. In conventional reactor physics methods, the gamma heating component is ignored such that the gamma absorption is forced to occur at the gamma emission site. For experimental reactor systems like EBR-II and FFTF, the placement of structural pins and assemblies internal to the core leads to problems with power heating predictions because there is no fission power source internal to the assembly to dictate a spatial distribution of the power.

As part of the EBR-II support work in the 1980s, the GAMSOR code was developed to assist analysts in calculating the gamma heating. The GAMSOR code is a modified version of DIF3D and actually functions within a sequence of DIF3D calculations. The gamma flux in a conventional fission reactor system does not perturb the neutron flux and thus the gamma flux calculation can be cast as a fixed source problem given a solution to the steady state neutron flux equation. This leads to a sequence of DIF3D calculations, called the GAMSOR sequence, which involves solving the neutron flux, then the gamma flux, and then combining the results to do a summary edit.

In this manuscript, we go over the GAMSOR code and detail how it is put together and functions. We also discuss how to setup the GAMSOR sequence and input for each DIF3D calculation in the GAMSOR sequence. With the GAMSOR capability, users can take any valid steady state DIF3D calculation and compute the power distribution due to neutron and gamma heating. The $\mathrm{MC}^{2}-3$ code is the preferable companion code to use for generating neutron and gamma cross section data, but the GAMSOR code can accept cross section data from other sources. To further this aspect, an additional utility code was created which demonstrates how to merge the neutron and gamma cross section data together to carry out a simultaneous solve of the coupled neutron and gamma transport equations.

Using the GAMSOR code, we demonstrate in this manual how the coupled neutron and gamma transport equations are solved simultaneously via an analytic benchmark problem. In addition, we use the GAMSOR code with $\mathrm{MC}^{2}-3$ on a series of two-dimensional verification problems to demonstrate its accuracy against MCNP. Finally, we show GAMSOR results for an EBR-II calculation also performed using MCNP. At present, validation using ZPPR TLD measurements are being carried out but were not available for inclusion in this manuscript. 


\section{TABLE OF CONTENTS}

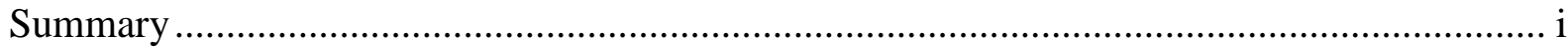

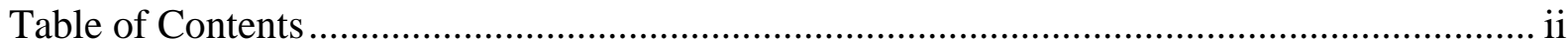

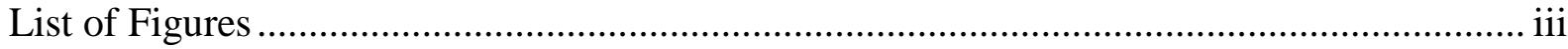

List of Tables ................................................................................................................. iv

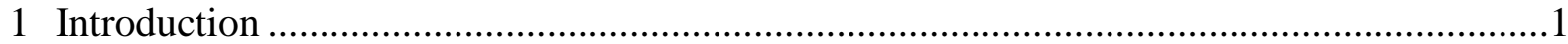

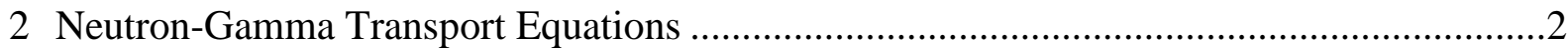

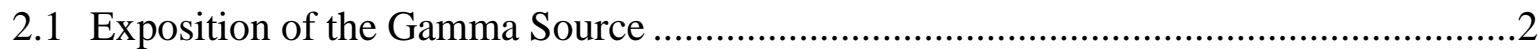

2.2 Power Normalization .............................................................................................

2.3 ARC System Treatment of Gamma Transport...............................................................6

3 Using the GAMSOR Code ………………....................................................................

3.1 $\mathrm{MC}^{2}-3$ Procedure for Generating Gamma Cross Sections..............................................

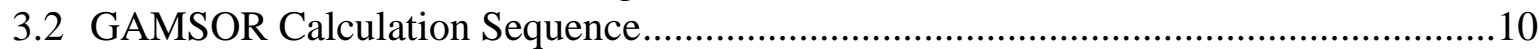

3.2.1 GAMSOR Step 1 Details ...........................................................................14

3.2.2 GAMSOR Step 2 Details ..............................................................................18

3.2.3 GAMSOR Step 3 Details ……………………….......................................19

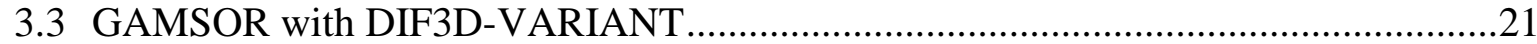

3.4 GAMSOR_Merge Utility Program........................................................................22

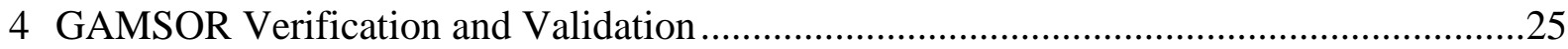

4.1 Simple GAMSOR Verification Problems........................................................................25

4.2 Two-Dimensional GAMSOR Verification Problems....................................................27

4.2.1 Unrodded Uranium Dominated Core Problem......................................................29

4.2.2 Rodded Uranium Dominated Core Problem ...........................................................36

4.2.3 Rodded Plutonium Dominated Core Problem....................................................39

4.3 EBR-II Verification Problems ………………………....................................................43

5 Gamma Library Generation with NJOY ………………………………………….........47

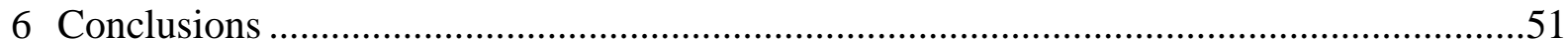

Appendix A. Quick Reference GAMSOR Guidance ………………........................................53

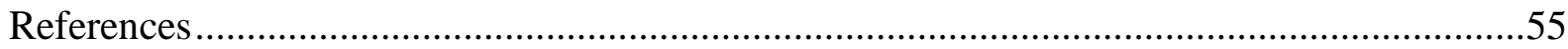




\section{LIST OF FIGURES}

Figure 3.1. $\mathrm{MC}^{2}-3$ Neutron and Gamma Cross Section Data Generation ...............................8

Figure 3.2. Typical DIF3D Input Using Binary Input Files............................................... 11

Figure 3.3. Typical DIF3D Input Setup and Output Results .............................................. 11

Figure 3.4. GAMSOR Execution Sequence with Primary Binary Files Listed ........................13

Figure 3.5. Example First GAMSOR Step DIF3D-FD Input ............................................ 15

Figure 3.6. Example First GAMSOR Step Output .......................................................... 17

Figure 3.7. Example Second GAMSOR Step DIF3D-FD Input .......................................... 19

Figure 3.8. Example Third GAMSOR Step DIF3D-FD Input..............................................20

Figure 3.9. Example Third GAMSOR Step DIF3D-FD Output of Interest.............................21

Figure 3.10. GAMSOR_Merge Execution Sequence ....................................................... 23

Figure 4.1. Simple Two-Dimensional Test Problem with Tally Region Numbering ...............28

Figure 4.2. Two-Dimensional Heating Distributions of Three Cases.....................................28

Figure 4.3. Configuration 1 Neutron Flux Spectrum Results for Tallies 1, 6, and 10.............31

Figure 4.4. Configuration 1 Neutron Flux Spectrum Results for Tallies 12, 14, and 17.........32

Figure 4.5. Configuration 1 Gamma Flux Spectrum Results for Tallies 1, 6, and 10. ............33

Figure 4.6. Configuration 1 Gamma Flux Spectrum Results for Tallies 12, 14, and 17.........34

Figure 4.7. Neutron and Gamma Heating Results for Configuration 1. ................................35

Figure 4.8. Deterministic Errors in Neutron and Gamma Heating Results for

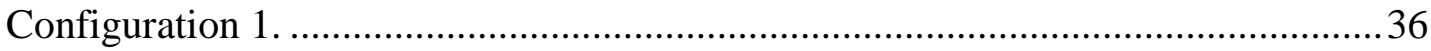

Figure 4.9. Neutron and Gamma Heating Results for Configuration 2. ................................37

Figure 4.10. Neutron Flux Results for Tallies 1, 9, and 16 of Configuration 2......................38

Figure 4.11. Gamma Flux Results for Tallies 1, 9, and 16 of Configuration 2. .....................38

Figure 4.12. Neutron and Gamma Heating Results for Configuration 3. ..............................40

Figure 4.13. DIF3D Core Center Gamma Flux Spectrum in Configurations 2 and 3.............41

Figure 4.14. Gamma Sources for Configurations 2 and 3 Plotted on Identical Scales............42

Figure 4.15. EBR-II Core Loading Pattern for Run 138B...................................................43

Figure 4.16. Power Difference (\%) for Inner-Core Assemblies between DIF3D and

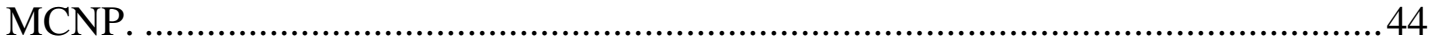

Figure 5.1. Generation of Gamma Libraries Using NJOY .............................................. 47 


\section{LIST OF TABLES}

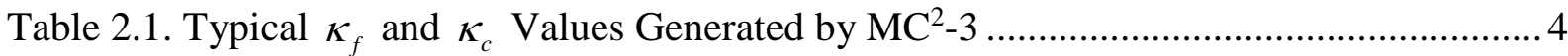

Table 4.1. Simple Verification Problem Cross Section Data................................................... 25

Table 4.2. Equivalent Two-Group Representation of the Simple Verification Problem.......... 26

Table 4.3. Material Compositions for the Configuration 1 Two-Dimensional Test

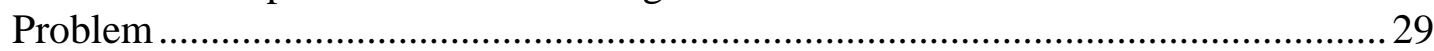

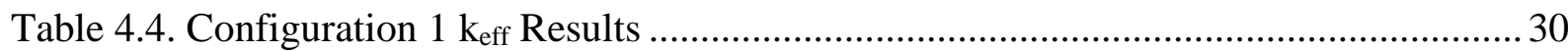

Table 4.5. Configuration 1 MCNP Neutron Flux and Statistical Errors for the $1^{\text {st }}$ Tally ......... 31

Table 4.6. Configuration 1 MCNP Neutron Flux and Statistical Errors for the $17^{\text {th }}$ Tally....... 33

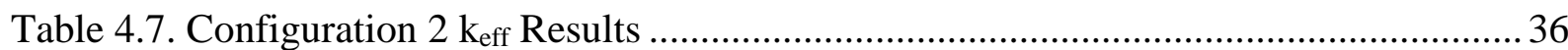

Table 4.8. Material Compositions for the Configuration 3 Two-Dimensional Test

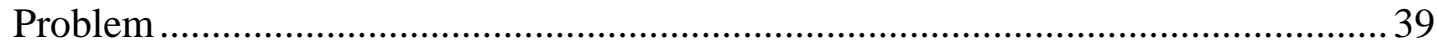

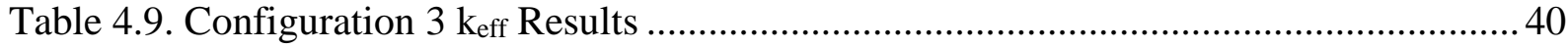

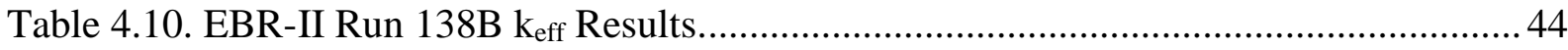

Table 4.11. Axial Power Density $\left(\mathrm{W} / \mathrm{cm}^{3}\right)$ in the XX10 Assembly .................................... 45

Table 4.12. Axial Power Density $\left(\mathrm{W} / \mathrm{cm}^{3}\right)$ in the High-Worth Control Assembly .................. 46

Table 5.1. Upper Energy Boundaries (eV) of the 21-group Structure of Gamma................... 49

Table 5.2. Structure of the Prompt Gamma Production Library of Each Isotope ................... 50

Table 5.3. Structure of the Gamma Interaction Cross Section Library of Each Isotope .......... 50

Table 5.4. Structure of the Index File table_gammalib ....................................................... 50

Table 6.1. GAMSOR Usage Quick Reference ............................................................... 53 


\section{Introduction}

Nuclear reactors that rely upon the fission reaction have two modes of thermal energy deposition in the reactor system: neutron capture and gamma absorption. The gamma rays are typically generated by neutron absorption reactions or during the fission process which means the primary driver of energy production is of course the neutron interaction. It should come as no surprise that the routine reactor analysis and design work does not consider the transmission of gamma rays but generally assumes some fraction of their energy will be converted to thermal energy in the reactor system.

For fuel cycle analysis, in particular the prediction of actinide transmutation, this approach is more than sufficient as the total energy production can be preserved. For other needs such as fuel performance, swelling, cladding embrittlement, radiation induced creep and power peaking, this approximation varies from inadequate to moderately inaccurate. As part of the full design process constructed by Argonne National Laboratory for sodium cooled fast spectrum reactors, the GAMSOR code was developed to properly handle the coupled neutron-gamma flux solution. In the GAMSOR sequence, the power density generation due to neutron and gamma interactions is produced, along with the total, for use in downstream tools such as mechanics and thermal hydraulics. In this sense it is a one-way coupling approach where any multi-physics feedback requires the input to the GAMSOR code to be updated. 


\section{Neutron-Gamma Transport Equations}

The DIF3D code [1-6] consists of multi-group diffusion and transport solvers including the finite difference option DIF3D-FD, the transverse integrated nodal option DIF3D-NODAL, and the even-parity transport option DIF3D-VARIANT. We can write the steady state multi-group neutron transport equation as

$$
\begin{aligned}
& \hat{\Omega} \cdot \nabla \psi_{g}(r, \hat{\Omega})+\Sigma_{t, g}(r) \psi_{g}(r, \hat{\Omega})=S_{g}(r, \hat{\Omega}), \\
& S_{g}(r, \hat{\Omega})=\sum_{g^{\prime}} \int d \Omega^{\prime} \Sigma_{s, g^{\prime} \rightarrow g}\left(r, \hat{\Omega}^{\prime} \rightarrow \hat{\Omega}\right) \psi_{g^{\prime}}\left(r, \hat{\Omega}^{\prime}\right) \\
& +\lambda \sum_{g^{\prime}} \chi_{g, g^{\prime}}(r) \cdot v \sum_{f, g^{\prime}}(r) \int d \Omega^{\prime} \psi_{g^{\prime}}\left(r, \hat{\Omega}^{\prime}\right)+Q_{g}(r, \hat{\Omega})
\end{aligned}
$$

The standard notation is used [7] where $\psi_{g}(r, \hat{\Omega})$ represents the neutron flux and $\Sigma_{t, g}(r)$ is the total interaction probability. The source is generally written separately as done in equation 2.2 which consists of the scattering source with interaction probability $\Sigma_{s, g^{\prime} \rightarrow g}\left(r, \hat{\Omega}^{\prime} \rightarrow \hat{\Omega}\right)$, fission source with interaction probability $\Sigma_{f, g^{\prime}}(r)$, and generic fixed source $Q_{g}(r, \hat{\Omega})$ which is generally only appropriate when fission is not present or $\lambda=0$.

The steady state gamma ray transport equation that is needed in the fission reactor can be written as

$$
\begin{aligned}
& \hat{\Omega} \cdot \nabla \Psi_{p}(r, \hat{\Omega})+\sigma_{t, p}(r) \Psi_{p}(r, \hat{\Omega})=s_{p}(r, \hat{\Omega}), \\
& s_{p}(r, \hat{\Omega})=\sum_{p^{\prime}} \int d \Omega^{\prime} \sigma_{s, p^{\prime} \rightarrow p}\left(r, \hat{\Omega}^{\prime} \rightarrow \hat{\Omega}\right) \Psi_{p^{\prime}}\left(r, \hat{\Omega}^{\prime}\right)+q_{p}(r, \hat{\Omega}) .
\end{aligned}
$$

To avoid notation confusion, we switch the lowercase and uppercase notation with respect to the neutron transport equation given in equation 2.1 and the group index $p$ is used instead of $g$ to further assist in defining the discrete transfer. $\Psi_{p}(r, \hat{\Omega})$ represents the gamma flux, $\sigma_{t, g}(r)$ is the total interaction probability, and the source $s_{p}(r, \hat{\Omega})$ consists of scattering with interaction probability $\Sigma_{s, p^{\prime} \rightarrow p}\left(r, \hat{\Omega}^{\prime} \rightarrow \hat{\Omega}\right)$ and a fixed source $q_{p}(r, \hat{\Omega})$ that needs further discussion.

\subsection{Exposition of the Gamma Source}

In equations 2.1 through 2.4, there is no clear indication of the linkage between the neutron and gamma transport equations because the source of gammas that drives equation 2.3, i.e. neutron interaction, is obscured in the source $q_{p}(r, \hat{\Omega})$. The most prominent source of gammas is the fission reaction which requires the gamma source to have the form:

$\lambda \sum_{g^{\prime}} X_{p, g^{\prime}}(r) \Sigma_{f, g^{\prime}}(r) \int d \Omega^{\prime} \psi_{g^{\prime}}\left(r, \hat{\Omega}^{\prime}\right)$. 
In equation 2.5, the neutron fission interaction probability is combined with the neutron flux to define the fission reaction rate. In theory, a neutron should yield a slightly different gamma ray distribution with respect to magnitude and spectrum based upon its initial energy which we indicate with the transfer matrix $X_{p, g^{\prime}}(r)$. Finally, the entire term must be scaled with $\lambda$ as the production rate of fission based gammas will be different than those of capture. In the steady state sense, the above $X_{p, g^{\prime}}(r)$ should contain both prompt and delayed gamma production components.

Excluding elastic scattering reactions, most of the neutron reactions will release some kind of a photon although the energy and importance of those photons might effectively be zero. Looking exclusively at capture reactions where a neutron is captured by the nucleus and gamma rays are emitted, we can write the source contribution as

$$
T_{p, g^{\prime}}(r) \Sigma_{c, g^{\prime}}(r) \int d \Omega^{\prime} \psi_{g^{\prime}}\left(r, \hat{\Omega}^{\prime}\right) \text {. }
$$

There is no integral over energy as the emitted gamma ray is a function of the incident neutron energy. We also note that there is no angular dependence of the gamma rays in either the capture reaction of equation 2.6 or the fission reaction of equation 2.5. This is consistent with the observed physics where capture of the neutron leads to random ejection of gamma rays with respect to direction.

The remaining principle reactions of interest, including inelastic scattering, can be written very similarly to equation 2.6 and thus we just express them all as

$$
\sum_{x} T_{x, p, g^{\prime}}(r) \Sigma_{x, g^{\prime}}(r) \int d \Omega^{\prime} \psi_{g^{\prime}}\left(r, \hat{\Omega}^{\prime}\right) \text {, }
$$

where $T_{x, p, g^{\prime}}(r)$ represents the neutron to gamma ray transfer matrix for neutron reaction $\Sigma_{x, g^{\prime}}(r)$. With this form we can rewrite equation 2.4 as

$$
\begin{aligned}
s_{p}(r, \hat{\Omega}) & =\sum_{p^{\prime}} \int d \Omega^{\prime} \sigma_{s, p^{\prime} \rightarrow p}\left(r, \hat{\Omega}^{\prime} \rightarrow \hat{\Omega}\right) \Psi_{p^{\prime}}\left(r, \hat{\Omega}^{\prime}\right)+\lambda \sum_{g^{\prime}} X_{p, g^{\prime}}(r) \Sigma_{f, g^{\prime}}(r) \int d \Omega^{\prime} \psi_{g^{\prime}}\left(r, \hat{\Omega}^{\prime}\right) \\
& +\sum_{x} T_{x, p, g^{\prime}}(r) \Sigma_{x, g^{\prime}}(r) \int d \Omega^{\prime} \psi_{g^{\prime}}\left(r, \hat{\Omega}^{\prime}\right)+q_{p}(r, \hat{\Omega})
\end{aligned}
$$

where $q_{p}(r, \hat{\Omega})$ now represents a fixed external source of gamma rays which can be independent of the neutron interaction (such as decaying actinides). For power reactor systems this external fixed source magnitude is generally not important and is neglected.

\subsection{Power Normalization}

One key feature of the above equations is that the flux magnitude requires an additional constraint (excluding sub-critical fixed source driven systems). The Argonne Reactor Code (ARC) system was built on the conventional approach that gamma ray transport was not important and as a consequence, the total power is computed using:

$$
P=\kappa_{f} \cdot \sum_{g} \int d V\left\{\Sigma_{f, g}(r) \int d \Omega^{\prime} \psi_{g}\left(r, \hat{\Omega}^{\prime}\right)\right\}+\kappa_{c} \cdot \sum_{g} \int d V \sum_{x}\left\{\Sigma_{x, g}(r) \int d \Omega^{\prime} \psi_{g}\left(r, \hat{\Omega}^{\prime}\right)\right\},
$$


where reactions " $x$ " consist of $(n, \gamma),(n, p),(n, d),(n, \alpha)$, and $(n, t)$ reactions, and $\kappa_{f}$ and $\kappa_{c}$ are the energy conversion factors due to fission and capture reactions, respectively. Starting with the fission conversion factor, $\kappa_{f}$, it includes not only the energy released as kinetic energy of the fission products, but the total energy of the gammas and other particles that are emitted in the fission process. Table 2.1 shows typical energy release values for the fissionable isotopes that $\mathrm{MC}^{2}-3$ [8] outputs. When converted to $\mathrm{MeV} /$ fission one can see these are consistent with the literature values of $220 \mathrm{MeV}$ energy release where $\sim 20 \mathrm{MeV}$ is lost to neutrino emission.

Table 2.1. Typical $\kappa_{f}$ and $\kappa_{c}$ Values Generated by $\mathrm{MC}^{2}-3$

\begin{tabular}{|c|c|c|c|c|}
\hline \multirow{2}{*}{ Isotope } & \multicolumn{2}{|c|}{$\kappa_{f}$} & \multicolumn{2}{c|}{$\kappa_{c}$} \\
\cline { 2 - 5 } & $\begin{array}{c}\text { Watts/fission } \\
\left(\cdot 10^{-11}\right)\end{array}$ & MeV/fission & $\begin{array}{c}\text { Watts/capture } \\
\left(\cdot 10^{-13}\right)\end{array}$ & MeV/capture \\
\hline U-235 & 3.100 & 193.5 & 10.486 & 6.54 \\
\hline $\mathrm{U}-238$ & 3.173 & 198.0 & 7.700 & 4.81 \\
\hline $\mathrm{Pu}-239$ & 3.186 & 198.8 & 10.468 & 6.53 \\
\hline $\mathrm{Pu}-240$ & 3.196 & 199.5 & 8.397 & 5.24 \\
\hline $\mathrm{Pu}-241$ & 3.236 & 202.0 & 10.095 & 6.30 \\
\hline
\end{tabular}

As one might infer, the fission and capture power conversion factors are provided with the ENDF data table and thus are just direct translations of the evaluated data. This is not the actual situation. The energy released by the $(n, \gamma)$ and $(n, d)$ reactions are fundamentally different and using a flat $\kappa_{c}$ to account for the energy conversion is not really physical. Thus equation 2.9 is an artifact of an approximation where the engineer understands that $100 \%$ of all of the energy from all reactions will be deposited somewhere in the reactor system and thus this representation is just simpler. It is also a by-product of the physical data that can be measured.

The $\kappa_{c}$ observed in Table 2.1 is actually obtained from an NJOY calculation of the energy release per reaction [9]. The KERMA (Kinetic Energy Release in MAterials) is obtained in NJOY via an "averaged" product of the flux and heating of a mixture.

$\operatorname{Heating}(E)=\sum_{i}^{\text {isotope reaction }} \sum_{j} N_{i} \kappa_{i, j}(E) \cdot \int d \Omega^{\prime} \psi\left(\hat{\Omega}^{\prime}, E\right)$.

In equation 2.10, $N_{i}$ is the atom density and $\kappa_{i, j}(E)$ is the KERMA for reaction $j$ of isotope $i$. NJOY computes the KERMA factors using the "energy-balance method" expressed as

$\kappa_{i, j}(E)=\left\{E+Q_{i, j}-E_{i, j, n}^{\prime}(E)-E_{i, j, \gamma}^{\prime}(E)\right\} \sigma_{i, j}(E)$.

In equation 2.11, $E$ is the energy of the incident neutron, $E_{i, j, n}^{\prime}(E)$ is the energy of the emitted neutron(s), $E_{i, j, \gamma}^{\prime}(E)$ is the energy of the emitted photon(s), and $Q_{i, j}$ is the reaction 
mass difference. The microscopic cross section $\sigma_{i, j}(E)$ is obviously represented continuously over the energy domain.

In the approach outlined in equation 2.11, the energy released per reaction of each isotope should be definable. Of course these KERMA factors require a normalization to the measured heating of the material as defined in equation 2.10. With regard to Table 2.1, it should be clear that the function $\kappa_{i \text {, fission }}(E)$ has been averaged over energy to construct an average quantity usable in the ARC system. Similarly, the "capture" related quantities were separated from the fission component, and averaged over energy to define another generic power conversion factor for the other reactions.

The primary issue with this technique is the inclusion of the cross section and flux in equation 2.10. The existence of these quantities implies that there is a spectrum weighting that might be inconsistent with the real problem. In the previous work [10-11], it has been observed that the $\kappa_{c}$ factor for iron decreased from 6.1 MeV/capture in the homogenized fuel assembly to $1.7 \mathrm{MeV} /$ capture in the homogenized shielding assembly. This is primarily due to a softening of the gamma ray spectrum and the resulting normalization inconsistencies that occur with applying equation 2.10. Again, in the ARC system all of the energy is assumed to be absorbed somewhere in the reactor domain and thus the gamma rays that are primarily generated in the fuel via fission are not transported and instead absorbed in the fuel. The neutron spectrum used to generate the $\kappa_{c}$ is very close to the actual spectrum that is observed in the fuel material. By taking this approach, $\mathrm{MC}^{2}-3$ and ARC can ignore the spatial and energy dependence of $\kappa_{f}$ and $\kappa_{c}$, thereby yielding a single value that is valid so long as all of the energy derived from neutron reactions is deposited somewhere in the reactor system.

The issue of course is that the spatial distribution of that energy deposition is rather invalid as it is known that 3-5\% of the total power is generated outside of the fuel assemblies. To capture the energy dependence of the neutron and gamma reactions, we alter the power computation to be

$$
\begin{aligned}
P & =\sum_{i} \sum_{x} \int d E\left\{\int d V \kappa_{x}(E) \cdot \Sigma_{i, x}(E) \int d \Omega^{\prime} \psi\left(\hat{\Omega}^{\prime}, E\right)\right\} \\
& +\sum_{i} \int d E\left\{\int d V K_{i}(E) \cdot \sigma_{i}(E) \int d \Omega^{\prime} \Psi\left(\hat{\Omega}^{\prime}, E\right)\right\}
\end{aligned} .
$$

In this case we define a generic gamma "capture" cross section to include the energy release rather than store individual reaction components. The gamma interaction KERMAs in equation 2.12 are then obtained using

$$
K_{i}(E)=E \cdot\left(\sigma_{i, \text { photo }}(E)+\sigma_{i, \text { compton }}(E)\right)+\{E-1.02 M e V\} \sigma_{i, \text { pair }}(E) .
$$

In equation 2.13 we see the three important gamma reactions: photoelectric, Compton scattering, and pair production. These can be separated to obtain an individual KERMA for each reaction, but that was not the focus of the work on using NJOY. The photoelectric reaction leads to electrons being released which are assumed to lead to localized energy deposition (i.e. pure absorption). The Compton scattering component is treated as an inelastic scattering operation where the energy recovered is the residual energy left with the nucleus. 
Pair production is more tricky. While the residual energy is assumed to be locally absorbed, the positron and electron annihilation can occur at another spatial location of considerable distance thereby resulting in important gamma ray emissions at another location. While it would be ideal to track every particle type to "exactly" obtain the power distribution, it is unrealistic given the lack of cross section data and computational expense associated with tracking multiple particles. Thus pair production is treated similarly to the neutron $(n, 2 n)$ scattering event at the location of the reaction to properly account for the increase in net gamma rays. The same normalization applied to equation 2.11 is required and thus equation 2.10 is again used [9].

Equation 2.12 is quite practical today and allows one to easily identify problems with the normalization process in equations 2.10 and 2.11. In this approach we require the storage of principle reaction cross sections for neutron and gamma ray data along with KERMA factors for each reaction type. It also allows the most accurate approach possible as maintaining the energy dependence of the KERMA factors reduces problems with weighting with a spectrum that is inconsistent with the real problem. In an ideal world, the KERMA calculation would be done in line with $\mathrm{MC}^{2}-3$ such that NJOY is not necessary.

Given equation 2.12, a quick study brings up a significant question about its accuracy for reactor systems. It is very typical of reactor analysis of fast reactors to ignore all regions past the first ring of shielding. From the above, this might result in a significant amount of gamma rays to be lost to the vacuum boundary condition. While this is generally true for considerably leaky systems, this is not generally relevant for large power systems and one can simply add additional layers of shielding to test out the impact of the normalization problems.

\subsection{ARC System Treatment of Gamma Transport}

As mentioned, equation 2.12 is quite straightforward to use and rather clear in its implementation. However, this was not deemed convenient for the development of GAMSOR and instead ARC uses the following form of power normalization

$$
P=\sum_{i} \int d E\left\{\int d V N_{i} \cdot \kappa_{i}(E) \int d \Omega^{\prime} \psi\left(\hat{\Omega}^{\prime}, E\right)\right\}+\sum_{i} \int d E\left\{\int d V N_{i} \cdot K_{i}(E) \int d \Omega^{\prime} \Psi\left(\hat{\Omega}^{\prime}, E\right)\right\},
$$

which collapses to the multigroup form of

$$
P=\sum_{g} \sum_{i}\left\{\int d V N_{i} \cdot \kappa_{i, g} \int d \Omega^{\prime} \psi_{g}\left(\hat{\Omega}^{\prime}\right)\right\}+\sum_{p} \sum_{i}\left\{\int d V N_{i} \cdot K_{i, p} \int d \Omega^{\prime} \Psi_{p}\left(\hat{\Omega}^{\prime}\right)\right\} .
$$

In this form, the microscopic cross sections of each reaction type are merged with the computed KERMA factors to directly yield a "power conversion” cross section at each spatial location (i.e. composition) given the neutron and gamma flux solution at that point. Instead of just using region independent KERMA factor storage for each reaction type, this approach requires the storage of isotope based KERMA factors for each "composition" in the system. With respect to storage this approach almost certainly requires more storage because fast spectrum systems generally contain more compositions than there are modeled isotope reaction types for a given isotope. However, this approach is straightforward as it just requires a simple vector operation to form a compositional power conversion factor followed by a vector-vector operation by mesh point to compute the power. 
It is important to note that this approach obscures the actual KERMA factors as the power conversion factor merges all reactions of a given isotope together. The origin of this approach is a result of the difficulty in setting up the linkage between an isotope based set and the existing DIF3D binary interface files. To do it properly requires additional isotope mapping information which was deemed an unnecessary change to DIF3D, and, based upon the coding practices of the time, was likely wise. In the compositional approach, one can just look up the same isotope alias chosen by the user and compute a "compositional" power conversion factor for use in the power computation of equation 2.14. 


\section{Using the GAMSOR Code}

Using the GAMSOR code in ARC requires the generation of neutron and gamma cross section data files which is handled with $\mathrm{MC}^{2}-3$ [8]. Given the cross section data files, the user must have already created a DIF3D input model typical of reactor analysis. The GAMSOR calculation itself amounts to a three-step calculation procedure with DIF3D as will be discussed. The output of the GAMSOR calculation is a set of binary power density files which, with the neutron and gamma flux files (RTFLUX and/or NHFLUX), give the coupled neutron and gamma flux solution to the problem. To begin, we start with the cross section generation procedure.

\section{1 $M C^{2}-3$ Procedure for Generating Gamma Cross Sections}

The $\mathrm{MC}^{2}-3$ code is primarily constructed to carry out a slowing down calculations on user provided compositional inputs to obtain the neutron spectrum. Both energy and spatial self-shielding can be handled in this process with the ultimate goal of generating effective broad group neutron cross section data. The bulk of the resonance treatment that is the focus of computational effort in $\mathrm{MC}^{2}-3$ is rather useless for the treatment of gamma ray cross section data. This should be rather apparent by a simple comparison of isotopic neutron and gamma cross section data plots.

Figure 3.1 shows the cross section generation procedure used by $\mathrm{MC}^{2}-3$ to produce neutron and gamma ray cross section data. The manual should be consulted for specific input details. In Figure 3.1 one can see that the base ENDF/B data is used in two separate paths to produce input data libraries of neutron and gamma cross section data. The generation of neutron data is well covered in the $\mathrm{MC}^{2}-3$ manual while the gamma generation data is relatively unspecified at this time and we defer to the documentation provided with each $\mathrm{MC}^{2}-3$ gamma library with regard to how the gamma cross section data was generated.

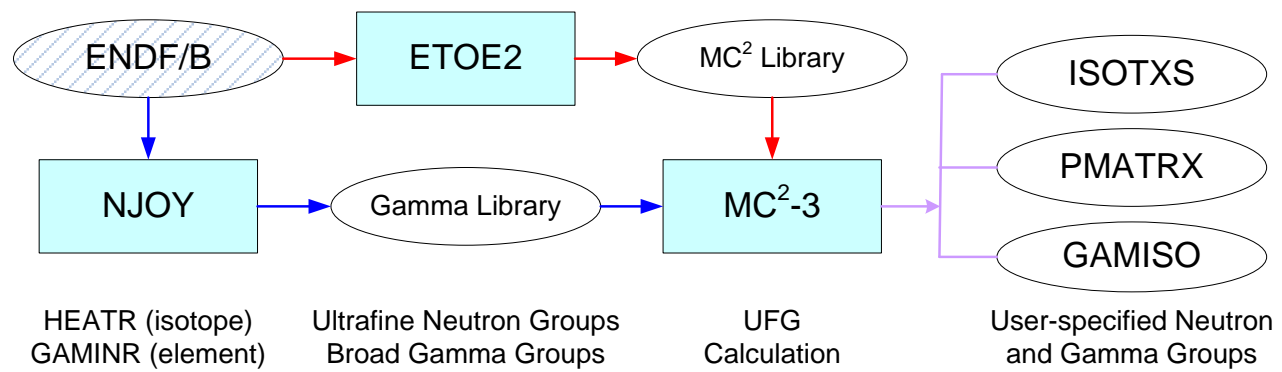

Figure 3.1. $\mathrm{MC}^{2}-3$ Neutron and Gamma Cross Section Data Generation

As discussed earlier, the ENDF/B cross section data is processed using NJOY to generate KERMA factors. The neutron KERMA factors computed with NJOY are merged with the ultra-fine group self-shielded neutron cross section data calculated in $\mathrm{MC}^{2}-3$ to produce the neutron power conversion factors. It is important to note that the KERMA factor generation scheme and the cross section generation schemes may be not consistent because the KERMA factors are generated from NJOY based on the infinite-dilute condition, which can lead to power conversion factors that do not necessarily agree with the expected physics behavior. 
In Figure 3.1 the gamma ray cross data is generated via NJOY and is linked to the self-shielded neutron cross section data obtained during the NJOY calculations (i.e. NJOY actually does both a neutron and gamma calculation). To allow it to be used generically in $\mathrm{MC}^{2}-3$, it is scaled on a group wise basis by the neutron cross sections obtained in the NJOY calculation. Upon completing the compositional neutron cross section data generation in $\mathrm{MC}^{2}-3$, the gamma cross section data from NJOY is scaled using the self-shielded neutron cross section data generated in $\mathrm{MC}^{2}-3$ to produce the correct reaction rate for the composition.

In Figure 3.1, one can see that three binary files are created upon completing the $\mathrm{MC}^{2}$ 3 calculation: ISOTXS, GAMISO, and PMATRX. The data file descriptions can be found in the appendices of the $\mathrm{MC}^{2}-3$ manual. The ISOTXS file contains the microscopic neutron cross section data for each isotope in each mixture. This ISOTXS file is constructed to be consistent with calculations that involve no gamma transport (the gamma emission sources are all neglected) such that the single energy power conversion factors given in equation 2.9 apply. The $(\mathrm{n}, \gamma)$ cross section is the reaction-based cross section for neutron capture and thus does not include the emission rate of gamma rays.

The GAMISO file is an ISOTXS formatted file. The typical neutron related reactions in GAMISO are never present (e.g. proton, alpha, deuteron, tritium are always zero). The (n, $\gamma$ ) cross section represents the absorptive loss of photons due to photon interactions with each isotope. The $(n, 2 n)$ cross section data, if present, is the pair production $(\gamma, 2 \gamma)$ which one should note will dominate at the upper energy regime of gammas. DIF3D will properly treat the $(\gamma, 2 \gamma)$ cross section data with regards to forming the DIF3D macroscopic cross section input (i.e. convert it to production form as needed). In some versions of $\mathrm{MC}^{2}-3$, the $(\gamma, 2 \gamma)$ component is not explicitly included and is instead included in the total (or elastic) scattering block of GAMISO in the production reaction rate form. This will make the capture cross section negative in order to allow the removal cross section to be computed correctly. The scattering data block contains the Rayleigh, Compton, and Thomson scattering components which is typically stored as elastic and $(n, 2 n)$. In those versions where pair production is not separated, all three components are included in the total scattering block in ISOTXS. The power conversion factors that are consistent with equation 2.9 are not provided in GAMISO which causes DIF3D to use a default value that is not accurate with respect to gamma power production. We therefore suggest not turning on the power edits during the fixed source gamma calculation.

Besides the two cross section data files, the PMATRX file from $\mathrm{MC}^{2}-3$ is the most essential component as it contains the neutron to gamma production based cross sections and the power conversion KERMA cross sections. The PMATRX can also contain dose conversion factors (rads/hour) for use with the neutron and gamma flux values calculated in DIF3D. The organization of data in the PMATRX file is done by isotope as in ISOTXS and GAMISO. For each isotope, the neutron heating cross section (eV-barns) along with the gamma heating cross section (eV-barns). The gamma production is given as a simple scattering matrix which can be expanded into spherical harmonic moments. $\mathrm{MC}^{2}-3$ does not currently compute any spherical harmonic expansion other than isotropic for gamma ray emission as the fission process does not indicate any preferential scattering behavior for gamma emission. It is important to note that the gamma production matrices are in the 
production form and thus will not sum to the $(n, \gamma)$ seen in the ISOTXS file. Instead, one must conceptually understand that the neutron equivalent " $v$ " has already been applied.

\subsection{GAMSOR Calculation Sequence}

The GAMSOR code is a modification of the standard DIF3D execution scheme and the DIF3D source code. To understand it, we must first review the standard DIF3D execution process and input which are graphically depicted in Figure 3.2 and Figure 3.3. Figure 3.2 shows an excerpt of the DIF3D ABR verification input where the DIF3D-VARIANT option is used.

The DIF3D code was originally organized as a series of separate executables which was focused on separating the major components of a neutronics calculation into individual tasks. The motivation of course was the limited computer memory at the time (1970s) where the executable instruction list took a considerable portion of the computer memory $(<64 \mathrm{~KB})$. Currently it is common to see a DIF3D executable size of $\sim 5 \mathrm{MB}$ and computer memory usage $>>32$ GB. Thus the old rules do not apply and the current DIF3D code is constructed as a single executable rather than numerous separate executables. The input of course has not significantly changed since the 1970s and thus we can see the artifact of having multiple executables.

The ARC system is organized into block operations such as load files, execute DIF3D, execute REBUS, execute VARI3D, etc. These control cards appear in Figure 3.2, on lines numbered 01 and 03 with the nomenclature "BLOCK" followed by the type of operation that is being performed. One can find the entirety of BLOCK control logic in reference [12] noting that not all of it is working currently because of the single executable setup. Under the "OLD" section of input (lines 01 and 02), one can see the declaration of the ISOTXS file as a "DATASET." This informs DIF3D that there is a binary file called ISOTXS that it can open and read cross section data from. One could also include the datasets FIXSRC and VARSRC as indicated at the top of Figure 3.3 which would inform DIF3D that those data files are also present. The ARC manual covers most of the different types of files that are supported by the ARC system, but for the purposes of GAMSOR, what is important to understand is that the binary data files PMATRX, RTFLUX, NHFLUX, PWDINT, COMPXS, GEODST, NDXSRF, ZNATDN, and LABELS can all be included in this section in the same manner. 


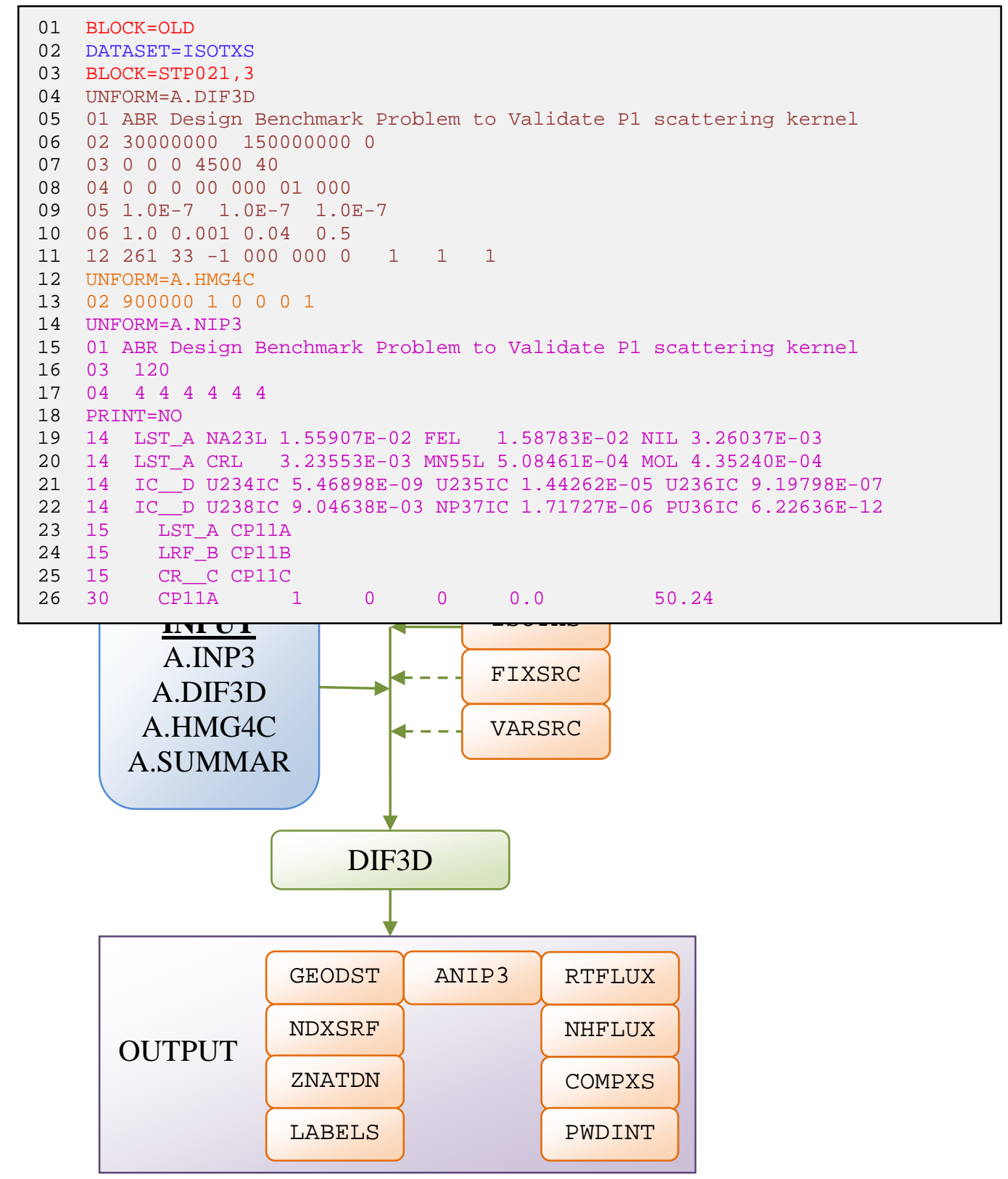

Figure 3.3. Typical DIF3D Input Setup and Output Results

In addition to the inclusion of binary files, one can also include ASCII files that are created during the "STP021" (and other such blocks) as seen in Figure 3.2 such A.NIP3, A.HMG4C, and A.DIF3D using the same "DATASET" descriptor. Of course one must have the corresponding files already processed by the ARC system present in the current working directory.

Under each block structure in Figure 3.2, one can see that the solver block "STP021" is broken into several components including A.DIF3D, A.HMG4C, and A.NIP3 which were all separate executables in the past. Each of these inputs has a specific purpose and output objective. Looking again at Figure 3.3, the successful execution of DIF3D yields several binary files in addition to the screen output. The GEODST (geometry), NDXSRF 
(composition isotope lists), ZNATDN (atom densities for each composition), and LABELS (region and composition labels) files are the equivalent representation of the A.NIP3 information and thus can be used as alternative inputs to A.NIP3 in executing DIF3D. In this case one would include these four files and ISOTXS in the old block setup. If one does not want the HMG4C branch of DIF3D to re-compute the macroscopic cross sections, stored in the binary file COMPXS, one can construct a DIF3D input only of GEODST, LABELS, and COMPXS although some aspects of the SUMMARY branch of DIF3D cannot be executed without NDXSRF, ZNATDN, and ISOTXS and thus SUMMARY can fail (A.SUMMAR input).

The other output files of importance in a regular DIF3D output are RTFLUX, NHFLUX, and PWDINT. The RTFLUX file contains the mesh averaged flux values (single scalar flux value per mesh point) which can be printed in a nice format using the CCCC_PrintTables.x utility program included in the src_utilities release of DIF3D. The NHFLUX file contains the space-angle polynomial expansion information by mesh point used in DIF3D-NODAL and DIF3D-VARIANT. The PWDINT file is the most important output for GAMSOR as it contains the average power density (watts $/ \mathrm{cm}^{3}$ ) by mesh point. In the standard DIF3D calculation, the PWDINT file will include the power density computed as if the gamma rays are absorbed where they are emitted. In GAMSOR, the PWDINT file will contain the merged power density from neutrons and gammas.

Figure 3.4 shows the primary execution path of the GAMSOR code. Because the neutron and gamma fluxes are not solved at the same time, the sequence requires three steps: neutron flux, gamma flux, and summary edit. The ISOTXS data structure does not allow energy dependent KERMA factors and thus merging the neutron and gamma cross section data into a single ISOTXS file cannot produce the same results as the sequence outlined in Figure 3.4. However, the COMPXS file does contain energy dependent power conversion factors and we have created a utility program in src_utilities called GAMSOR_Merge.x which can merge the neutron ISOTXS, the gamma GAMISO, and PMATRX files into a single COMPXS file. That capability is detailed in Section 3.4 of this report. 


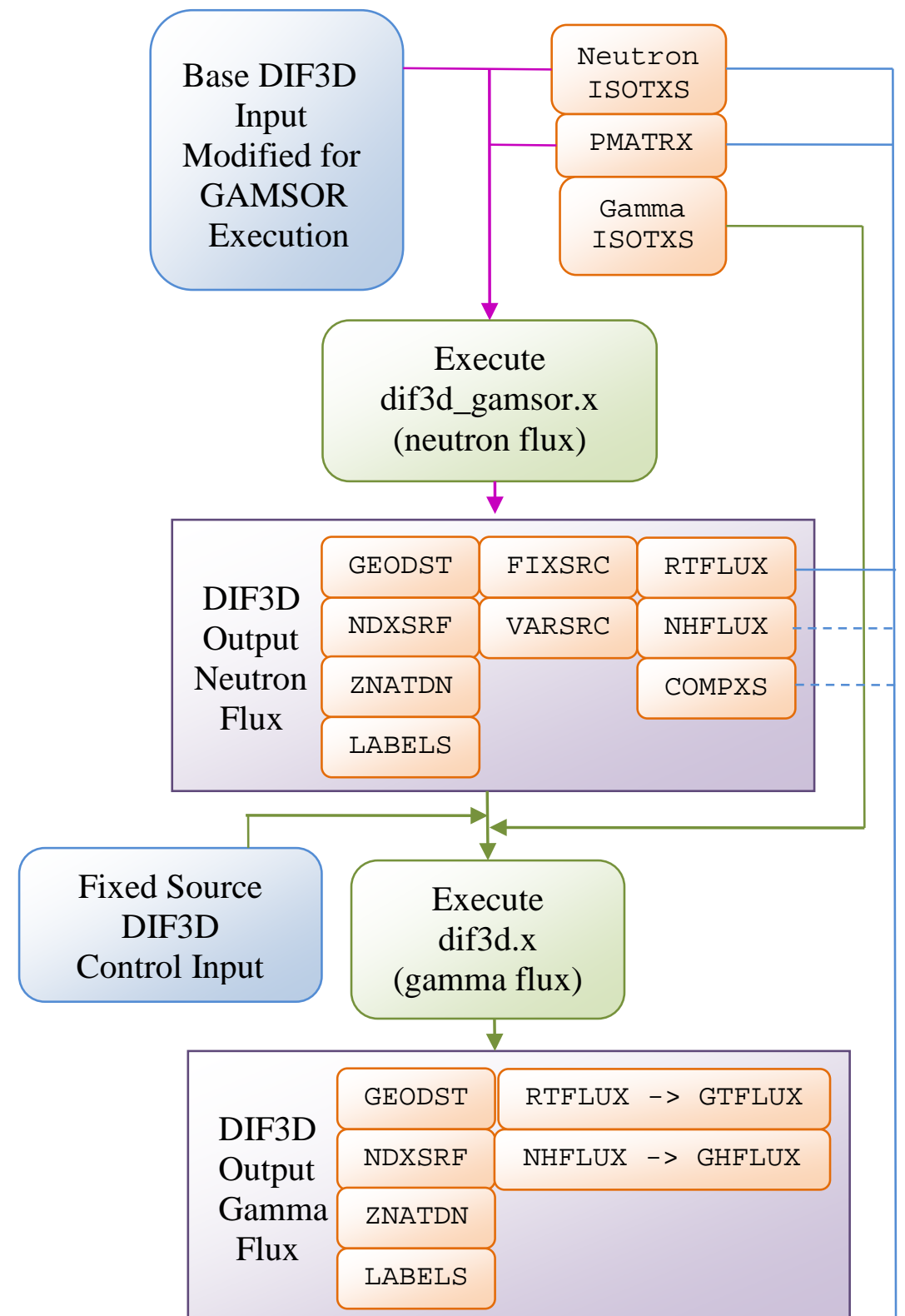

Figure 3.4. GAMSOR Execution Sequence with Primary Binary Files Listed

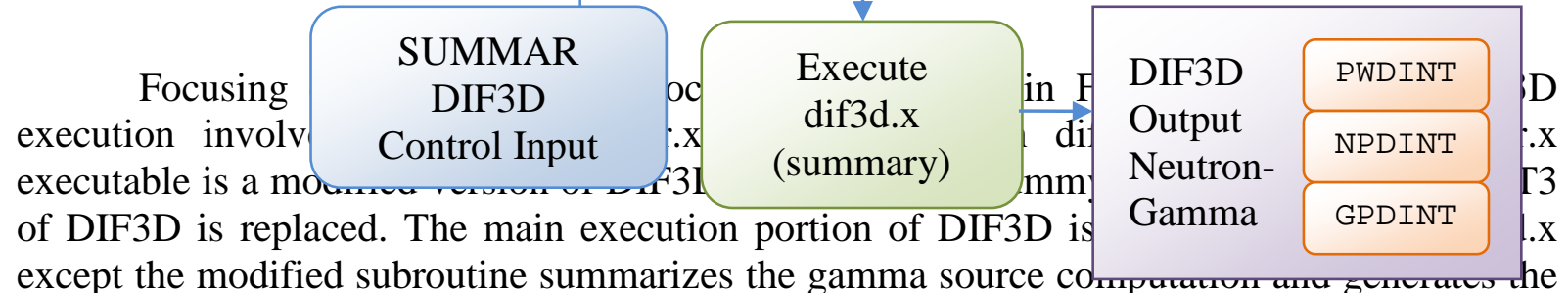
fixed source files FIXSRC and VARSRC. The FIXSRC file was originally meant for use with DIF3D-FD and thus provides a single flat source value per energy group per mesh point. Currently GAMSOR can be used using DIF3D-FD with no troubles other than the accuracy issues of using diffusion theory. VARSRC was added more recently to systematically improve the accuracy of GAMSOR by allowing transport to be used when computing the neutron and gamma flux levels. The accuracy of the DIF3D-Nodal option of DIF3D will 
suffer compared with DIF3D-VARIANT and DIF3D-FD as it is restricted to using a FIXSRC file that is not representative of the actual neutron flux shape. The impact on a test problem will be shown later in addition to a validation case using EBR-II measured data.

After each step of GAMSOR in Figure 3.4, the user is required to maintain some binary interface files which are required in the final summary step and rename others. Starting with the completion of dif3d_gamsor.x, the user must maintain the RTFLUX file where the NHFLUX and COMPXS are cited to be optional. The RTFLUX file is the primary flux storage file format for DIF3D-FD and is required to carry out all edit options of DIF3D. The NHFLUX file is the DIF3D-VARIANT flux solution and is considered optional as it is only needed to evaluate the polynomial flux and reaction rates at point locations throughout the domain using the utility code EvaluateFlux.x. One can store NHFLX0 as a smaller sized (scalar flux only) alternative to the NHFLUX file. The COMPXS file stores the homogenized cross sections for each region in the domain. It is optional because DIF3D will simply recompute it in the third step if it is not present and is needed. After the second step, the RTFLUX file that is generated by DIF3D must be renamed as GTFLUX to avoid a conflict with the RTFLUX file from the first step. Similarly, one must rename the NHFLUX file to avoid a conflict with the stored neutron NHFLUX file from the second step.

\subsubsection{GAMSOR Step 1 Details}

In the first step of Figure 3.4, the binary files ISOTXS and PMATRX are required along with the ASCII inputs A.DIF3D, A.HMG4C and A.NIP3. The ASCII inputs are similar to Figure 3.2 and numerous examples can be found in the verification test suite of DIF3D that comes with each release. The primary modification to Figure 3.2 is the inclusion of the PMATRX file, two examples of which are shown in Figure 3.5 for the DIF3D-FD option. To use DIF3D-VARIANT, one needs to add a card type 12 in the A.DIF3D section of input similar to line 11 seen earlier in Figure 3.2. As can be seen in Figure 3.5, the PMATRX file is now included as a "DATASET" declaration in the "OLD" block of DIF3D input. In the second option, the restart option of DIF3D-FD is demonstrated assuming that the user had already obtained a flux solution for the neutron flux problem previously. In this approach the dif3d_gamsor.x code is only being used to obtain the gamma fixed source associated with the flux solution. 
In both

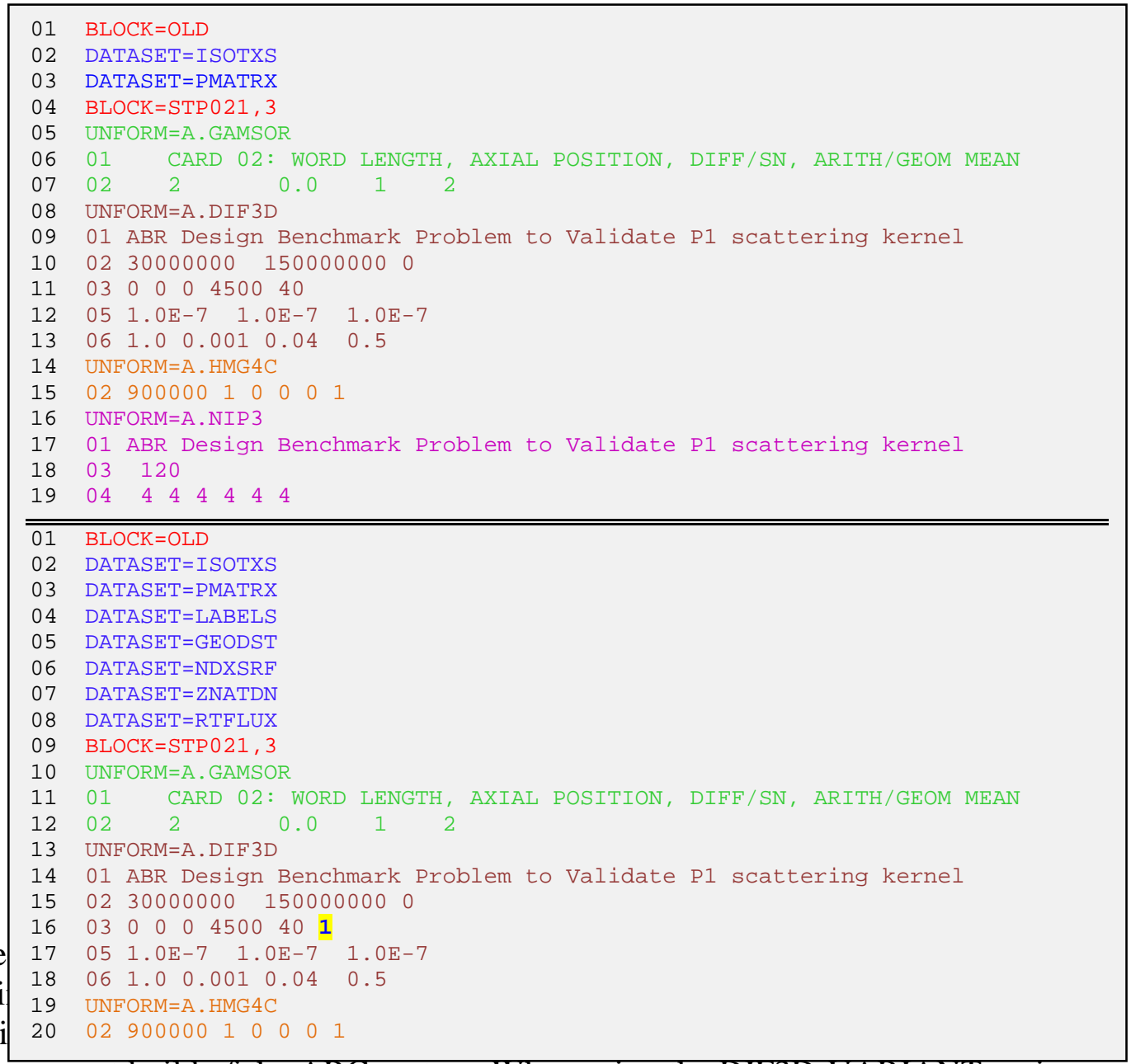

A.GAMSOR se options consisti input as it speci must be 2 in the current build of the ARC system. When using the DIF3D-VARIANT option, this input option is entirely ignored and unnecessary. The second A.GAMSOR input, a 0.0, specifies the axial mesh used to base a 2D source on. This technique was intended for generating a 2D gamma source for 2D transport codes at the time as 3D diffusion was deemed too inaccurate to use. The A.GAMSOR third input, a 1, is the primary means to trigger the 3D diffusion (i.e. DIF3D) versus a 2 to trigger a 2D source creation. It is not clear whether the 2D source creation option is really supported as the destination code is no longer available. The final A.GAMSOR input, a 2, is an antiquated choice of producing erroneous gamma power output. One can choose a 1 or a 2 where the first computes the average gamma energy within an energy group by averaging the energy boundaries. The second utilizes the square root of the boundaries to come up with a "more accurate" average which is generally suggested because of the way the photons are distributed. Both approaches are just inaccurate ways of computing the actual gamma power done correctly in the third GAMSOR step and we thus suggest ignoring the gamma power output in this section.

Given that the first step of GAMSOR is a regular neutron flux problem, the standard output is provided where capture is defined as $(\mathrm{n}, \gamma)$ earlier. The GAMSOR coding will always generate a FIXSRC file and, if using DIF3D-VARIANT, a VARSRC file also. In addition, there will be output edits from GAMSOR as shown in Figure 3.6 for a very simple problem. 
The first section of GAMSOR output details the control variables used for GAMSOR along with the title which is the only indication that the GAMSOR routines are being used. In this case a 33 group neutron problem is being combined with a 21 group gamma problem. As mentioned above, the A.GAMSOR controls are no longer relevant today. One should note that the energy conversion factor of $1.6023 \cdot 10^{-13}$ (Watt-sec/MeV) is slightly wrong with respect to today's value of $1.602176487 \cdot 10^{-13}$. The actual value used in the GAMSOR coding is exactly as seen above although this conversion factor appears elsewhere in the ARC system as $1.6022 \cdot 10^{-13}, 1.60219 \cdot 10^{-13}$, and $1.60207 \cdot 10^{-13}$ all of which were valid at one point during the ENDF evaluation process. We defer to fixing these discrepancies to future work as the output derived from using this factor should be ignored anyway.

The second section of output from GAMSOR lists the average gamma ray energy for each energy group defined in the GAMISO file. These values are not obtained from the average neutron velocities provided in GAMISO but are, as indicated, computed using the "geometric" average. These rather suspect numbers are used to compute the "gamma power" shown in the fourth section of output in Figure 3.6.

The third set of GAMSOR output should never appear as it indicates missing gamma production cross section data in the $\mathrm{MC}^{2}-3$ library. One should always check for this output and contact ANL staff using nera-software@anl.gov if this output appears as it can indicate a serious problem with the gamma library that is being used. In this example, the Mo-100 gamma production data was missing in the ENDF library and the GAMSOR output verifies that the checking algorithm detects it. It is important to note that the Mo-100 gamma production data is also reported missing in the MCNP [24] code when it executes. 


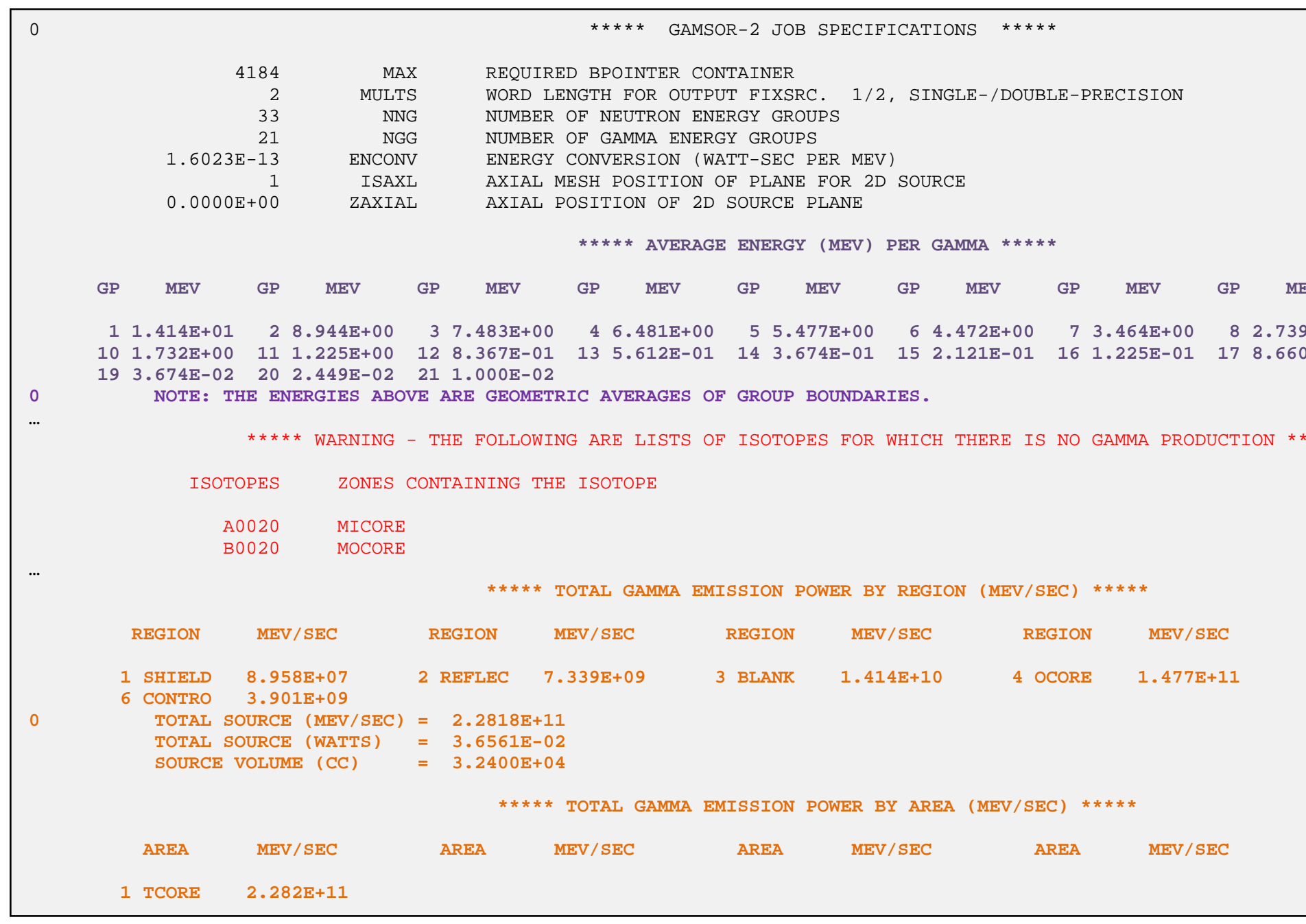


The fourth section of GAMSOR output lists the region-wise gamma source information in units of $\mathrm{MeV} / \mathrm{sec}$ which is obviously an integral over energy using the average gamma energies mentioned above. This data is inaccurate but it provides a reasonable way to determine the regions that are producing the bulk of gammas and give relative magnitude type of information and acts as a sanity check of the GAMSOR process. The area edit sums are also given for convenience.

\subsubsection{GAMSOR Step 2 Details}

As indicated in Figure 3.4, it is essential that the user maintain the RTFLUX and FIXSRC (and/or VARSRC) files from the first step of GAMSOR. The second step of GAMSOR has input similar to the first step where we give two examples in Figure 3.7. The first example is the suggested option where the A.NIP3 data is repeated either using ASCII input or by including the DATASET in the old block as shown. The second input option can also be used, but it requires the GAMISO file to contain the identical set of isotopes seen in ISOTXS in the exact same order. We have observed that users generally create more isotopes in the neutron ISOTXS file for use with reactivity coefficients whereas the GAMISO file only contains the isotopes needed for standard operation. In this circumstance, the list of isotopes in NDXSRF is no longer consistent with the list in GAMISO and rather invalid gamma flux values result. It is therefore strongly suggested that the A.NIP3 dataset be included or that the A.NIP3 input be repeated in step 2 of GAMSOR. It is important to note that one has to rename the GAMISO file as ISOTXS during this step.

Other than the removal of the A.GAMSOR input, the only other substantial changes from Figure 3.5 are the modification to the error tolerance inputs and inclusion of a 1 on card type 03 (lines 9 and 12). The card type 03 modification tells DIF3D that this is a fixed source problem and not a eigenvalue problem. The error tolerance changes (lines 11 and 14) are a consequence of the way DIF3D was constructed. DIF3D never contained a rigorous fixed source solver as it was almost entirely focused on eigenvalue problems. Consequently there is no check of the flux error between "outer" iterations, only the fission source and eigenvalue. In a fixed source gamma calculation, this poses quite the problem as a single Gauss-Seidel iteration in energy is needed to converge where the inversion of the diagonal (within-group space-angle system) should be very precise. Without a check on the flux error, there is no indication from DIF3D as to the accuracy that was achieved on the gamma flux calculation. Worse, the inversion of the within-group system is generally set with an error tolerance of 0.04 which is appropriate for an eigenvalue problem. In line 11 of the first example of Figure 3.7 we have highlighted the third input of the card type 6 A.DIF3D. This input corresponds to the within-group flux solve relative error tolerance to achieve during the Gauss-Seidel iteration in energy. Unlike the 0.04 value seen in Figure 3.5, the Figure 3.7 input is using $10^{-7}$ which is the only way to control the error on the within group flux solution in DIF3D-FD.

During the DIF3D-FD setup, the number of iterations required to invert the withingroup system and achieve this specified error is determined for a fictitious source distribution. During the real solve, the fixed number of iterations is used and the within-group flux error should generally be close to the input specified above. As mentioned there is no actual error information provided on the computed gamma flux and thus one has to literally repeat the 
calculation with a different input setting and manually error check the flux solution to determine whether convergence was achieved. In most cases of running GAMSOR with DIF3D-FD, using $10^{-7}$ yields sufficiently converged gamma flux values. The DIF3DVARIANT solver acts differently from DIF3D-FD and the approach required for a fixed source is discussed in a later section.

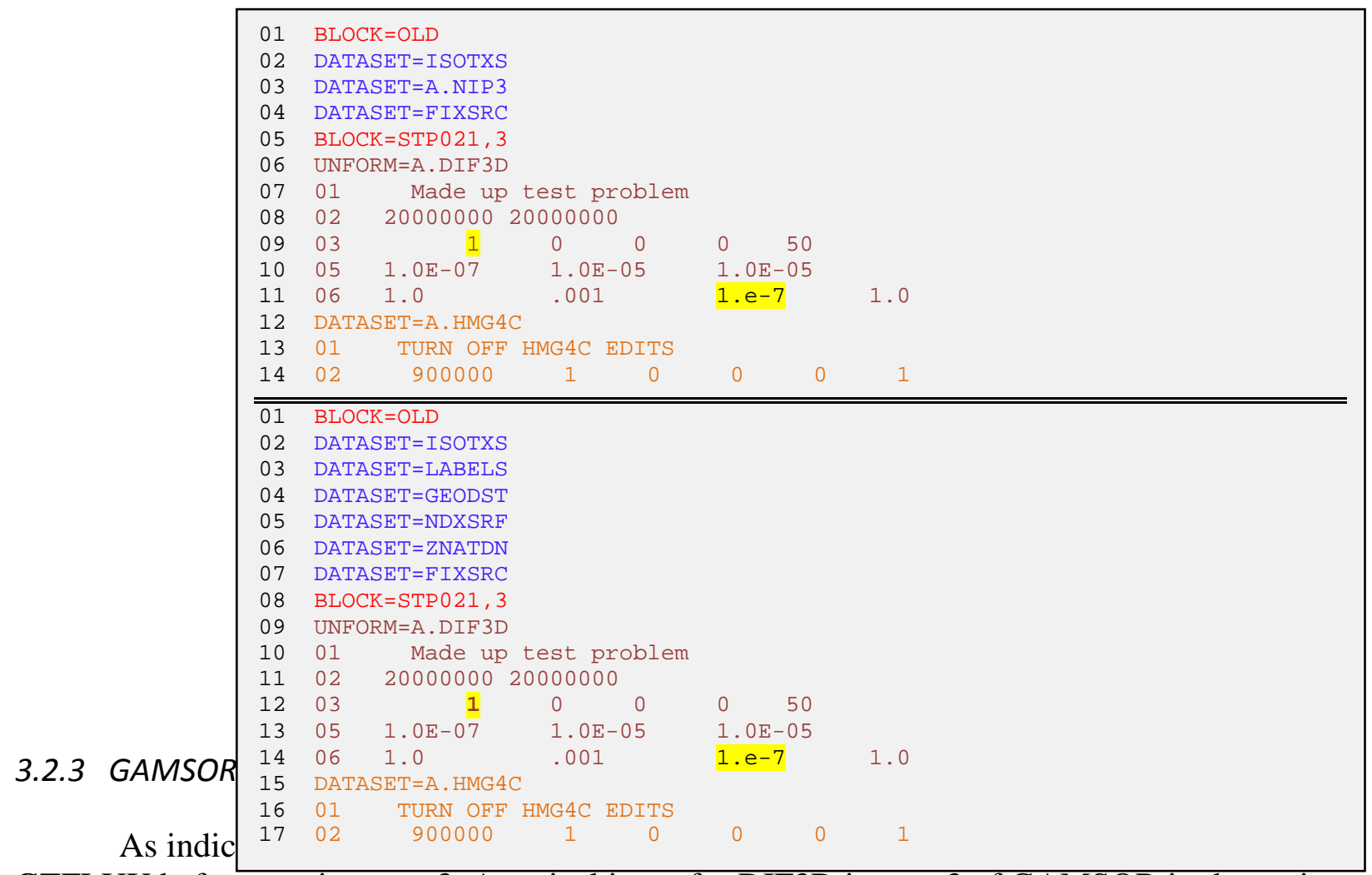

GTFLUX before starting step 3. A typical input for DIF3D in step 3 of GAMSOR is shown in Figure 3.8. In this case we again see the inclusion of PMATRX in the OLD block section of input. Additionally, we see the gamma flux file, GTFLUX file is included in the OLD block along with the neutron flux file RTFLUX. Looking at the A.DIF3D input, one can see the removal of all inputs except for card type 3 which only has a non-zero at position 1 and a -3 at position 5 . The first 1 is required in order to trigger the correct edits. The -3 simultaneously prevents DIF3D from solving the steady state eigenvalue problem or doing any type of neutronics edits. If one attempts to use -2, one will obtain the neutronics edits in the conventional DIF3D approach where power is computed using equation 2.9 rather than equation 2.14. This is why the card type 4 input is dropped from A.DIF3D in step 3 as the output will be identical to step 1 if enabled.

The three binary output files shown as outputs of the third GAMSOR step in Figure 3.4 are created as a part of the SUMMARY module of DIF3D. This is the reason for the A.SUMMAR inclusion in Figure 3.8. The inputs to A.SUMMAR are not relevant to the creation of the three binary files as it will automatically be done so long as RTFLUX, GTFLUX, and the PMATRX are all included as data files. The bulk of the SUMMAR output that is sent to the screen is only based upon the neutron flux results. Thus it is identical to what would be seen in step 1 using the same input except for one key section shown in Figure 
3.9. In this output only lines 2 and 4 are modified from the original neutron flux related quantities. Line 2 is the notification statement that the neutron and gamma heating factors from the PMATRX file will be used to compute the mesh wise power. Line 4 shows the outcome of the test problem to produce 0.461 watts of power.

The primary issue of course is that 0.461 watts is not consistent with the 0.5 watt power setting chosen on line 6 of Figure 3.5. This amounts to an 8\% error in the user specified power level. To understand this, the power is normalized to the user specified 0.5 watts in step 1 using equation 2.9, where all released gammas are assumed to be absorbed. Depending upon the amount of gamma leakage from the modeled domain, one can understand that without an additional normalization of the neutron and gamma flux, the power computed in the third step will generally be less than that of the first step (typically 2-10\%). While one can attempt to vastly increase the size of the shielding regions to improve the energy deposition in the modeled domain, with respect to equation 2.14, it is sufficient to just modify the original input such that the power in step 3 appropriately matches the desired user power. Keep in mind that there are additional errors in the normalization of the neutron and gamma heating vectors constructed for equation 2.14 (and for equation 2.9) and what is really desired is that the modeled domain produce the correct amount of power.

Overall,

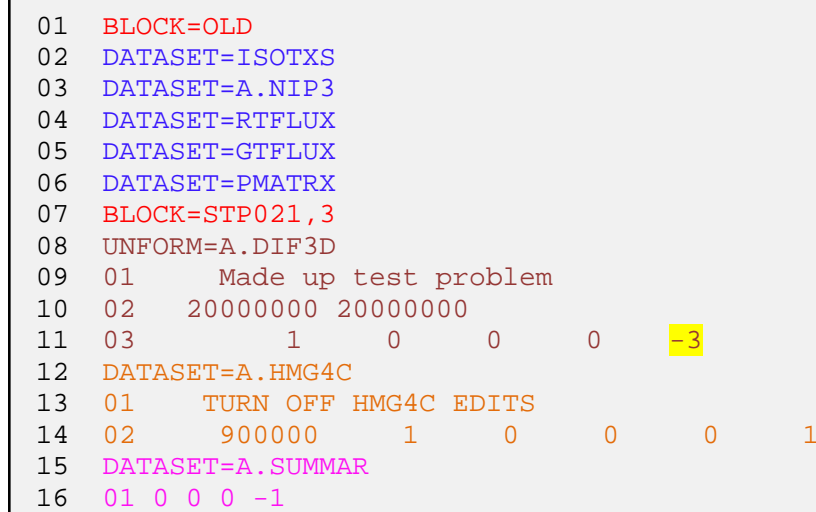

NPDINT, GPDINT, and PWDINT files and the two lines of modified output in the SUMMARY output as indicated in Figure 3.9. The NPDINT file is the neutron heating power component of equation 2.14 while the GPDINT file is the gamma heating power component of equation 2.14. The PWDINT file is the combined result of GPDINT and NPDINT. The RTFLUX file (and NHFLUX) from the first step gives the mesh wise neutron flux while the RTFLUX file (and NHFLUX) from the second step gives the mesh wise gamma flux distribution. 


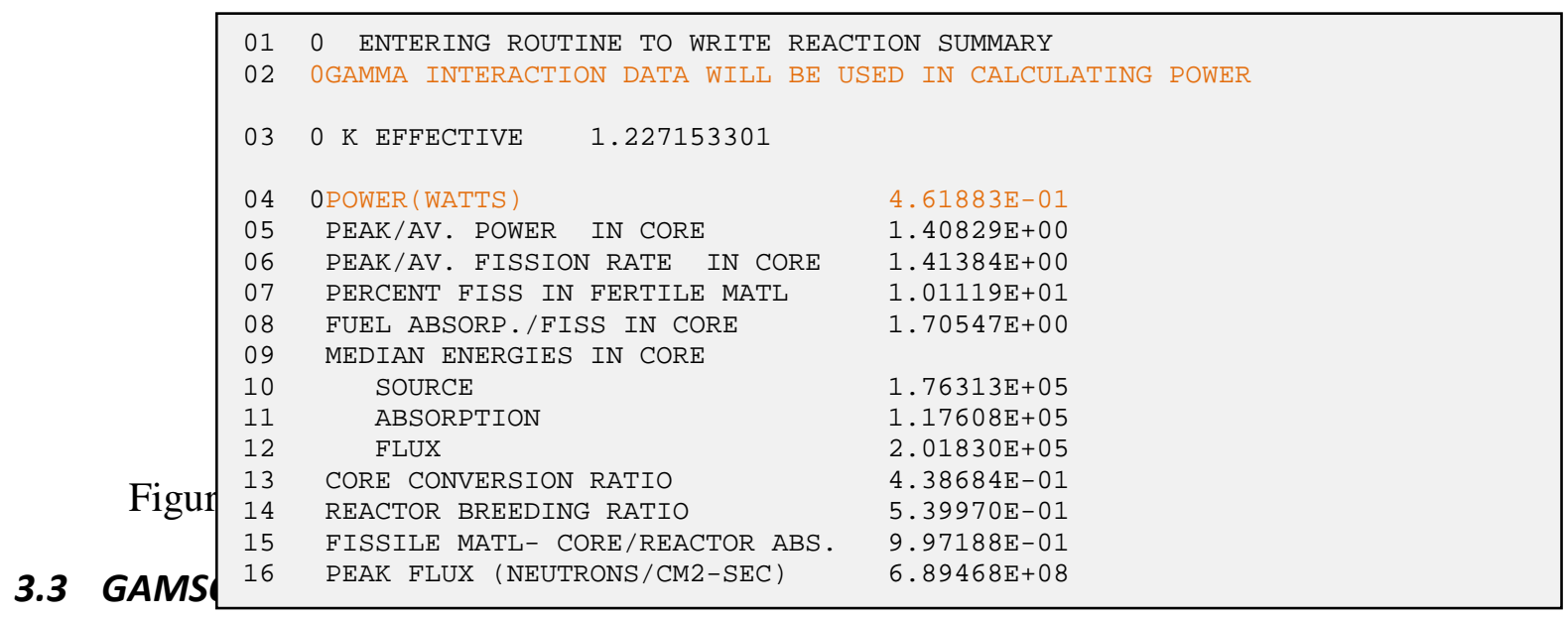

For DIF3D-NODAL and DIF3D-VARIANT, the FIXSRC file that is generated by step 1 in GAMSOR will still work, but this is not accurate as the polynomial dependence of the gamma source is completely lost. The use of FIXSRC amounts to a coarsening of the spatial approximation and effectively redistributes the gamma source over the mesh. For DIF3D-FD, one can trivially refine the mesh and observe how the gamma source distribution impacts the solution but for DIF3D-NODAL and DIF3D-VARIANT that approach is not straightforward, if even possible (hexagonal) as both solvers become overly expensive as the mesh is refined. For certain gamma energies, this can introduce a significant error in the solution as the axial distribution of the gamma rays generally pushes the gamma rays further away from the core and allows them to leak more from the system.

To counter the flat source approximation issue, the VARSRC file was introduced which retains the polynomial distribution of the source chosen in step 1 to define the gamma fixed source in step 2 of GAMSOR. This makes the three step procedure in Figure 3.4 equivalent to that of the merged system without the loss in accuracy that occurs when FIXSRC is used. The VARSRC file is only created when the DIF3D-VARIANT option is employed while the FIXSRC file is always created. The VARSRC file is only valid for DIF3D-VARIANT and will not be used with DIF3D-NODAL. The VARSRC was added to DIF3D over 3 years ago for the PERSENT code development [13] and has seen rigorous testing. It allows a full space-angle distribution of the gamma source even though the gamma emission from fission is isotropic. The real goal of course was to allow users to determine how much error was being introduced by using the diffusion approximation on the gamma flux calculation versus transport.

To execute DIF3D-VARIANT as part of GAMSOR, one needs to understand that the RTFLUX components are required to carry out the mesh wise edit process while NHFLUX actually stores the flux solution. To evaluate reaction rates within each mesh, one can use the EvaluateFlux.x utility program provided with the DIF3D release. In this utility code, the basis functions used to define the flux approximation are evaluated within each DIF3D-VARIANT mesh at the specified set of user points and the microscopic and macroscopic cross sections are evaluated. This type of interpolation is far more accurate and efficient compared with using the RCT based interpolation methods developed for DIF3D-NODAL [14]. The RTFLUX files generated using DIF3D-VARIANT can be directly compared with those of 
DIF3D-FD to understand the impact of using transport versus diffusion to model a given reactor system.

With respect to the fixed source calculation of GAMSOR, DIF3D-VARIANT does not make use of the card type 06 tolerance errors show in Figure 3.7, lines 11 and 14. Instead, one must manually set the number of iterations used via the card type 12 inputs. DIF3DVARIANT typically computes the quantities using an optical thickness rule over each plane (radial iterations) and all planes (axial iterations). DIF3D-VARIANT will typically select between 2 and 5 radial iterations and 2 and 3 axial iterations for most fast spectrum systems such that the error reduction per axial iteration is 0.1 . For fixed source problems, we must increase this to nearly 20 radial iterations and 10 axial iterations. In some extreme cases to eliminate negative fluxes in the periphery of the core, users have had to use 50 radial and 50 axial iterations. The unfortunate problem with both DIF3D-FD and DIF3D-VARIANT fixed source problems is that there is no guarantee what the achieved flux errors are going to be and thus users always have to run the problem twice with different convergence settings to verify that the desired convergence is met.

\subsection{GAMSOR_Merge Utility Program}

Figure 3.4 and the preceding discussion on GAMSOR output should indicate that the GAMSOR process is rather complex and that its output edits are rather minimal and can be erroneous (power computation in the second step). As part of the verification process we created the GAMSOR_Merge.x utility program that will merge the ISOTXS, GAMISO, and PMATRX files together.

Figure 3.10 shows the execution sequence required to use the utility code. As can be seen, the user input is required in the standard binary form which requires a null execution of DIF3D. Given the GEODST, NDXSRF, LABELS, and ZNATDN files, the GAMSOR_Merge.x utility code will produce a COMPXS and ISOTXS file which merges the neutron and gamma ISOTXS files and PMATRX. As mentioned previously, the ISOTXS file cannot store the required energy dependent conversion factors in equation 2.14. The COMPXS file does store the energy dependent conversion factors which were previously obtained by multiplying the fission cross section by the isotope energy conversion factor and the "capture" cross sections by the isotope capture energy conversion factor. Given the required storage is provided in COMPXS, we can simply form the macroscopic cross sections and take the conversion factors from the PMATRX rather than use the neutron conversion factors in the ISOTXS file. 


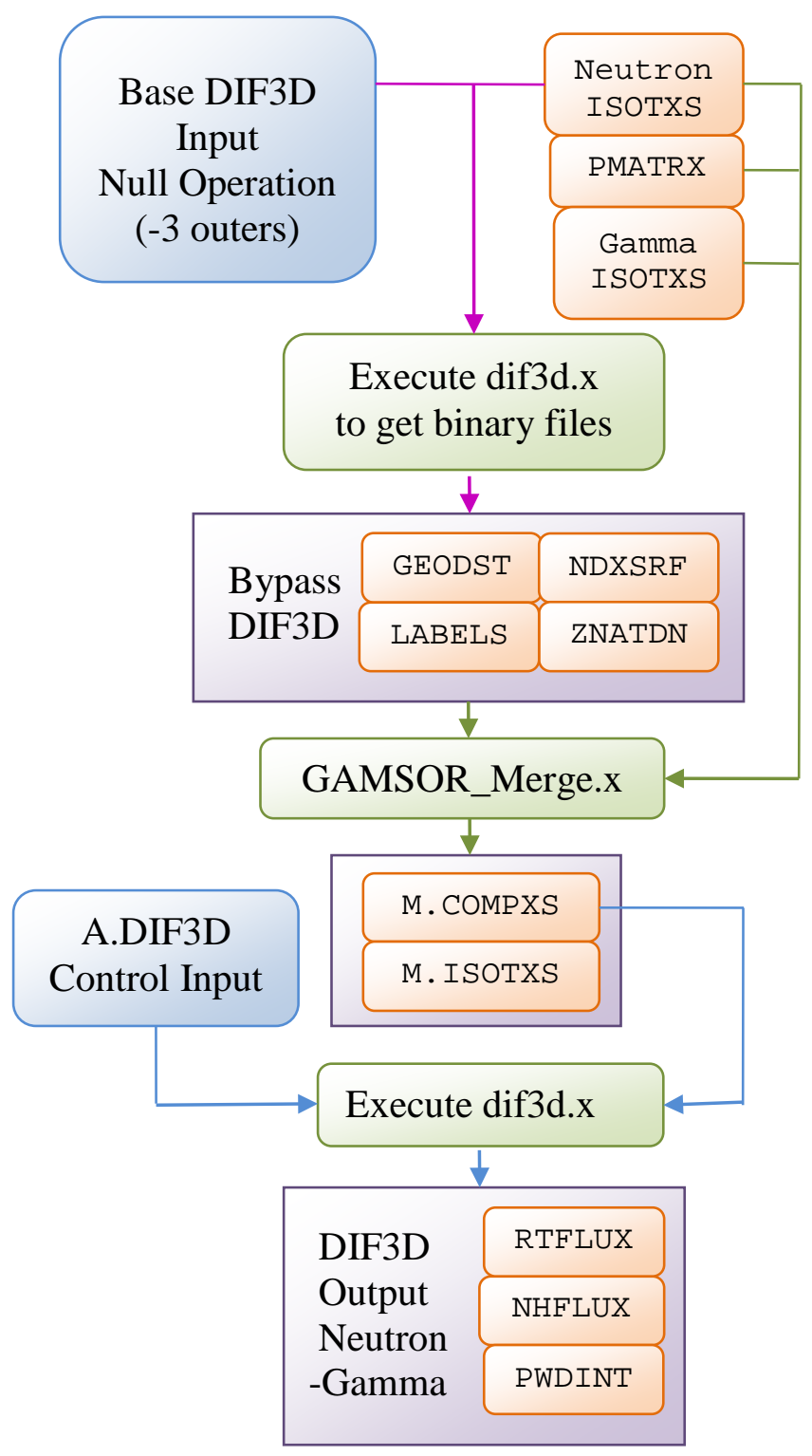

Figure 3.10. GAMSOR_Merge Execution Sequence

The problem with this approach of course is that the gamma flux calculation typically requires a single Gauss-Seidel iteration in energy (sweep through energy groups) to obtain the gamma flux where each within-group equation (diagonal) should be converged to $10^{-7}$. In the conventional eigenvalue solver, the gamma flux groups are still solved, but generally only achieve an effective flux error of $10^{-4}$ because of the noise filtering down from the neutron flux groups during the eigenvalue solve. This means that one has to tighten the fission source tolerance and reduce the tolerance on the flux error achieve on the within-group system (in DIF3D-VARIANT this means manually setting a higher number of iterations). While the within-group system does not have to be converged to $10^{-7}$, the typical value for DIF3D-FD of 0.04 is clearly insufficient and one should find that at least $10^{-4}$ is required. 
In Figure 3.10, the last dif3d.x execution requires input similar to Figure 3.7 except for the fixed source setup. The output is a RTFLUX file and NHFLUX file that can be used for regular flux edits noting that they will contain the neutron and gamma flux files. The ISOTXS file that is generated by GAMSOR_Merge.x can be used with SUMMARY noting that the isotopic reaction rates have been manipulated. The gamma "capture" has been placed into the $(n, d)$ position where the $(n, d)$ data for neutrons was merged with $(n, p)$. The $(n, 2 n)$ reaction cannot be moved without modifying the cross section balance. It is important to note that the ISOTXS file cannot be used to run the problem because the power normalization will be completely erroneous in addition to the problems with computing a proper removal cross section. 


\section{GAMSOR Verification and Validation}

Validation of gamma radiation emission in nuclear reactors is rather complex given the problems associated with separating measurements of neutron and gamma radiation. Nevertheless, we have the thermoluminescent dosimeter (TLD) measurements of neutron and gamma radiation measurements from several ZPPR experiments that can be used for validation in reactor systems [16-17]. Unfortunately those problems were not completed at the time of this report and we defer that type of validation work for later inclusion. Consequently, in this report we focus on the verification with simplistic test problems to show how the GAMSOR process operates and follow it with comparative calculations with MCNP [24].

\subsection{Simple GAMSOR Verification Problems}

The easiest way to verify GAMSOR is to construct and run a simple verification check. For this problem we manually created the ISOTXS, GAMISO, and PMATRX files. We used a one group structure for neutrons and one group structure for gammas where the base cross section data is given in Table 4.1. The $\Sigma_{\gamma p r o d}$ cross section in this form represents the production rate of gamma rays and represents the linkage between neutrons and gammas that is defined in the PMATRX. To reproduce the PMATRX operation, one should take the neutron reactions that lead to the production of gamma rays (fission, alpha, proton, gamma, etc.) and merge the gamma emission rate (energy and number of photons) with the neutron cross section to obtain this number.

Table 4.1. Simple Verification Problem Cross Section Data

\begin{tabular}{|c|c|c|c|c|c|c|}
\hline & $\Sigma_{\text {total }}$ & $\Sigma_{\text {fission }}$ & $\Sigma_{\text {capture }}$ & $v$ & $\Sigma_{\text {scatter }}$ & $\Sigma_{\gamma \text { prod }}$ \\
\hline Neutron & 1.0 & 0.5 & 0.5 & 10 & 0.0 & 0.5 \\
\hline Gamma & 1.0 & & 0.9 & & 0.1 & \\
\hline
\end{tabular}

In the form of Table 4.1, we simply provide the combined result which happens to exactly match the $\Sigma_{\text {capture }}$ cross section. So we can assume that $\Sigma_{\text {fission }}$ yields no photons whereas

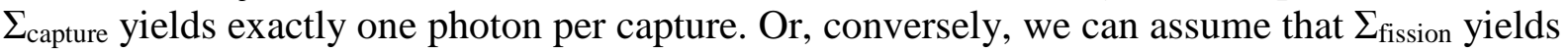

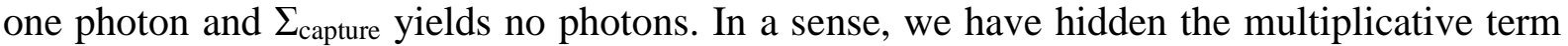
that is associated with how many gamma rays are produced from the fission and capture processes which is consistent with the PMATRX definition where no information about photons emitted per neutron absorbed is provided.

With regard to solving a coupled system of equations definable with the data in Table 4.1, we immediately have an issue. In the typical eigenvalue problem we can write

$$
\underline{\underline{A}} \cdot \underline{\psi}=\frac{1}{k_{\text {eff }}} \underline{\underline{F}} \cdot \underline{\psi} \cdot
$$

In this form, $\underline{\underline{F}}$ represents the discretized fission source operator (a matrix), $\underline{\psi}$ represents the neutron flux vector and $\underset{\underline{A}}{=}$ represents the streaming, collision, and scattering operators. In the conventional steady state mode, the fission source operator is separated from the other reactions and scaled with the eigenvalue $k_{\text {eff }}$ to produce a time stable system. The power is then computed by multiplying the steady state flux vector in equation 4.1 by all power 
producing neutron reactions and scaling the result to match the desired power. We can expand equation 4.1 to considered a coupled neutron and gamma problem and obtain

$\left[\begin{array}{cc}\underline{A}_{n} & 0 \\ -\underline{P}_{n, \gamma} & \underline{\underline{A}}\end{array}\right] \cdot\left[\begin{array}{l}\underline{\psi}_{n} \\ \underline{\psi}_{\gamma}\end{array}\right]=\frac{1}{k_{e f f}}\left[\begin{array}{cc}\underline{F} & 0 \\ \underline{P}_{f, \gamma} & 0\end{array}\right] \cdot\left[\begin{array}{l}\underline{\psi}_{n} \\ \underline{\psi}_{\gamma}\end{array}\right]$

Initially, this form might appear absurd as we have scaled the gamma production from fission with $k_{\text {eff }}$. In general, an operating reactor is effectively critical and thus $k_{\text {eff }}$ should be unity and writing the above itself is rather pointless. In reality, a computed $k_{\text {eff }}$ is rarely unity and there is some error that we typically decide is due to cross section data or geometry uncertainty. Thus from a modeling point of view, we typically cast $k_{\text {eff }}$ as an error in $v$ (neutrons per fission) even though it is just as likely to be an error in the relative amount of fission, capture, or leakage. Looking at the gamma production, an error in the neutron $v$, given the total energy emission requirement should remain constant, should translate to an error in the number of gammas emitted per fission. While the form of equation 4.2 might not properly relate the necessary photon energy scaling, it is included in this section to indicate that one must be careful when attempting a validation calculation that the gamma and neutron flux levels might not be scaled properly given the bias in the computed $k_{\text {eff }}$. (As an example, k-eff for a EBR-II diffusion calculation is typically off by 3\% and by using equation 4.2 we would adjust the gamma emission from fission, rather than $v$, to get the proper gamma power contribution.)

For fuel cycle analysis of steady state reactors systems, equation 4.2 is entirely pointless and, if applied, would most certainly be inaccurate. For this reason, both GAMSOR and MCNP properly interpret the two systems being connected as

$$
\left[\begin{array}{cc}
\stackrel{A}{=}_{n} & 0 \\
-\underline{P}_{n, \gamma}-\underline{P}_{f, \gamma} & \underline{A}_{\gamma}
\end{array}\right] \cdot\left[\begin{array}{l}
\underline{\psi}_{n} \\
\underline{\psi}_{\gamma}
\end{array}\right]=\frac{1}{k_{\text {eff }}}\left[\begin{array}{cc}
\underline{F} & 0 \\
0 & 0
\end{array}\right] \cdot\left[\begin{array}{l}
\underline{\psi}_{n} \\
\underline{\psi}_{\gamma}
\end{array}\right]
$$

This form is obviously more practical and assumes the only errors are due to $v$. As a final note on this matter, most Monte Carlo based codes do not presently track the gamma emission history, but only the gamma emission sites. The gammas that are actually created are sampled from all neutron reactions that emit gammas at those emission points rather than the actual neutron interactions that occurred. It is an open question as to whether this technique makes any meaningful impact on the types of problems being studied in this report.

We can recast the one group systems into a two group neutral particle problem of the form consistent with equation 4.3 as seen in Table 4.2 .

Table 4.2. Equivalent Two-Group Representation of the Simple Verification Problem

\begin{tabular}{|c|c|c|c|c|c|c|c|}
\hline \multirow{2}{*}{ Group } & \multirow{2}{*}{$\Sigma_{\text {total }}$} & \multirow{2}{*}{$\Sigma_{\text {fission }}$} & $\Sigma_{\text {capture }}$ & \multirow{2}{*}{$v \cdot \Sigma_{\text {fission }}$} & \multirow{2}{*}{$\sum_{\text {removal }}$} & \multicolumn{2}{|c|}{$\Sigma_{\text {scatter }}$} \\
\cline { 6 - 8 } & & & & & & $1 \rightarrow$ & $2 \rightarrow$ \\
\hline 1 & 1.0 & 0.5 & 0.5 & 5 & 1.0 & 0.0 & 0.0 \\
\hline 2 & 1.0 & 0.0 & 0.9 & 0.0 & 0.9 & 0.5 & 0.1 \\
\hline
\end{tabular}


One can easily see that the gamma production appears as a down scattering component of in $\Sigma_{\text {scatter. }}$ The balance applied to each microscopic cross section generally requires that the total cross section be equal to the sum of principle reactions and the out scatter block of data for each group. In this form, the reaction form of $(n, 2 n)$ (i.e. probability of interaction) used rather than the production based. In Table 4.2, one can see that the first energy group balance is off by 0.5 due to the gamma production that appears in the scattering block. This is not a concern for the macroscopic treatment as the $(n, 2 n)$ is typically stored in the production form in the scattering matrix and one almost always finds an imbalance.

Assuming an infinite homogeneous composition and total fission rate of 1.0, we can compute the eigenvalue to be 5.0, the neutron flux solution will be $2.0 \mathrm{n} / \mathrm{cm}^{2} \cdot \mathrm{s}$ and the gamma flux will be $1.111 \overline{1} \mathrm{~g} / \mathrm{cm}^{2} \cdot \mathrm{s}$. Applying the same limits to Table 4.1 one finds the identical solution. The purpose of the verification problem is to demonstrate how the two data sets can be merged into a single data set and produce identical solutions. The various problems that make up the verification problem number 4, are done to verify that the assembly of the two data sets works for multiple geometry options of DIF3D.

\subsection{Two-Dimensional GAMSOR Verification Problems}

Because of the computational effort required to get MCNP to reduce the statistical error on the neutron and gamma flux tallies for a problem like EBR-II, we choose to include a new verification problem which MCNP should be able to easily solve. For all of these tests we utilized MCNPX version 27b with ENDF/B-VII.0 cross sections which is consistent with the cross sections available in $\mathrm{MC}^{2}-3$ and MCNP version cited in the report [24]. In this problem we also demonstrate the accuracy differences between using the standard $\mathrm{MC}^{2}-3$ cross section generation and the 2D core calculation concept.

To begin, we show the 2D core geometry given in Figure 4.1 with the boundary conditions and dimensions indicated in the left picture where the right hand picture shows the selected tally regions used in MCNP. As indicated, there are two core material regions, a blanket, a reflector, and a shielding region. A control rod region is added in the outer core region to introduce an additional gradient.

We have setup three configurations based upon this geometry. The first one is a uranium dominated core region where the control rod region is filled with outer core material (case 1). The second configuration repeats case 1 with the control rod inserted (case 2) while the third configuration repeats case 2 but uses a plutonium dominated core rather than uranium one. The intention of course is to assess how much impact the two isotopic changes have on the accuracy of the power distribution.

Figure 4.2 shows the power distribution predicted in each configuration using DIF3DFD in GAMSOR. As can be seen, there are substantial differences in the power distribution and the introduction of the control rod substantially perturbs the power shape. In the uranium cases, there is a substantial enrichment difference between the inner and outer core leading to a dramatic discontinuity in power production while in the plutonium configuration there is not as much of one and one can see a rather smooth power distribution over the core region. 
Combined, these problems should allow us to assess how well $\mathrm{MC}^{2}-3$ and DIF3D via GAMSOR are handling the coupled neutron, gamma reactor problems.

The typical way to use $\mathrm{MC}^{2}-3$ is to run each composition as an infinite homogenous problem and generate a broad group library for use in DIF3D. In this regard we choose to use a standard 33 group structure for neutrons and 21 group structure for gammas [8]. A more advanced approach for generating cross sections is to construct a $2 \mathrm{D}$ representation of the 3D problem and use $\mathrm{MC}^{2}-3$ coupled with TWODANT to improve the region-wise cross sections. Of course in this target problem, we can directly run a $2 \mathrm{D}$ problem in $\mathrm{MC}^{2}-3$ and identically match the desired problem. In this case, we should be able to get the 1042 group flux solution in $\mathrm{MC}^{2}-3$ and given the region averaging, produce a nearly identical result using 33 groups in DIF3D.
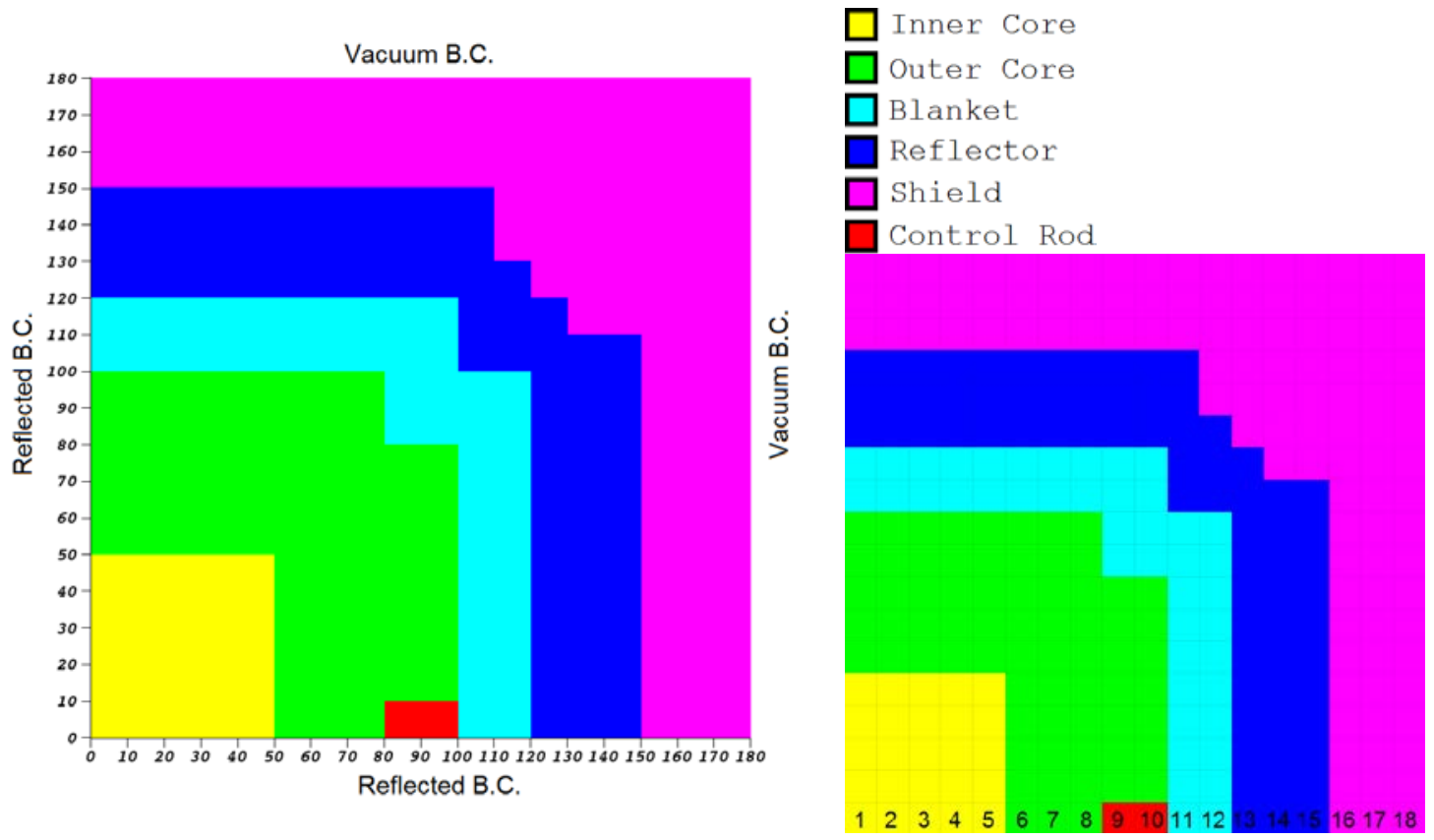

Figure 4.1. Simple Two-Dimensional Test Problem with Tally Region Numbering

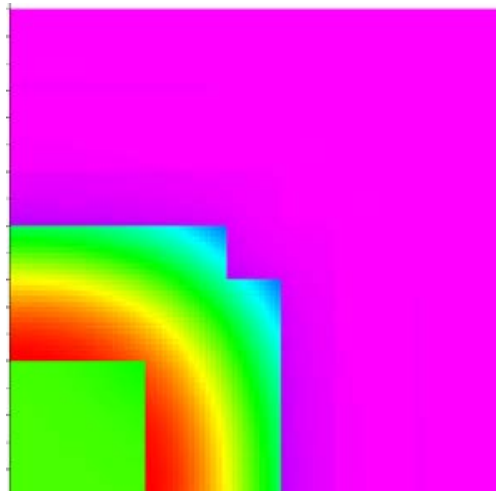

Unrodded Uranium

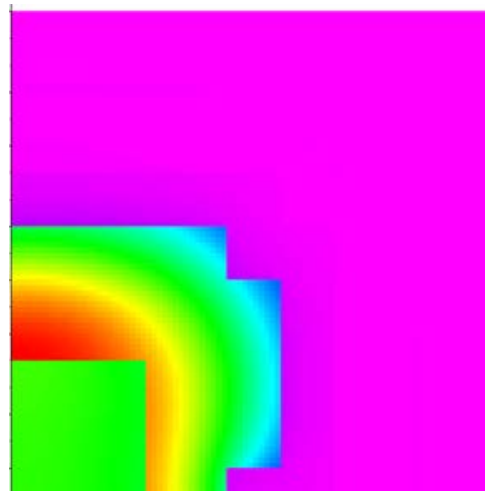

Rodded Uranium

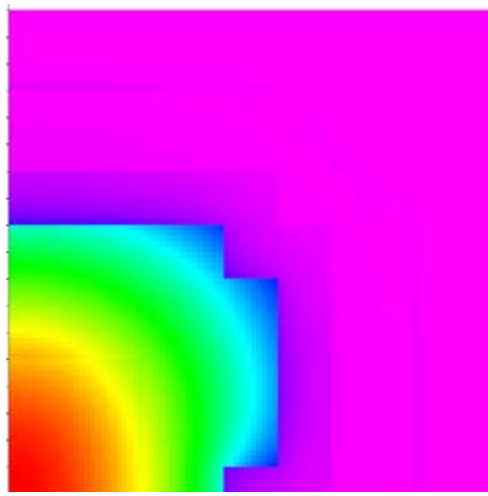

Rodded Plutonium

Figure 4.2. Two-Dimensional Heating Distributions of Three Cases 


\subsubsection{Unrodded Uranium Dominated Core Problem}

The compositions for the first configuration are given in Table 4.3 which consists of a standard homogeneous mixture for sodium cooled fast reactors with zirconium alloyed fuel. We note that the fuel content was made up for this problem and is thus quite simple consisting of just two uranium isotopes.

Table 4.3. Material Compositions for the Configuration 1 Two-Dimensional Test Problem

\begin{tabular}{|c|c|c|c|c|c|c|}
\hline Isotopes & Inner Core & Outer Core & Blanket & Reflector & Shield & Control Rod \\
\hline CR-50 & $1.0461 \mathrm{E}-04$ & $1.0461 \mathrm{E}-04$ & $1.0461 \mathrm{E}-04$ & 3.8036E-04 & $1.3720 \mathrm{E}-04$ & $1.2112 \mathrm{E}-04$ \\
\hline CR-52 & 2.0173E-03 & 2.0173E-03 & 2.0173E-03 & 7.3349E-03 & $2.6458 \mathrm{E}-03$ & $2.3356 \mathrm{E}-03$ \\
\hline CR-53 & $2.2874 \mathrm{E}-04$ & $2.2874 \mathrm{E}-04$ & $2.2874 \mathrm{E}-04$ & $8.3172 \mathrm{E}-04$ & $3.0001 \mathrm{E}-04$ & $2.6484 \mathrm{E}-04$ \\
\hline CR-54 & $5.6939 \mathrm{E}-05$ & $5.6939 \mathrm{E}-05$ & $5.6939 \mathrm{E}-05$ & 2.0703E-04 & $7.4679 \mathrm{E}-05$ & $6.5924 \mathrm{E}-05$ \\
\hline $\mathrm{NI}-58$ & $6.7965 \mathrm{E}-05$ & $6.7965 \mathrm{E}-05$ & $6.7965 \mathrm{E}-05$ & $2.4712 \mathrm{E}-04$ & $8.9140 \mathrm{E}-05$ & $7.8689 \mathrm{E}-05$ \\
\hline $\mathrm{NI}-60$ & $2.6180 \mathrm{E}-05$ & $2.6180 \mathrm{E}-05$ & $2.6180 \mathrm{E}-05$ & $9.5192 \mathrm{E}-05$ & $3.4337 \mathrm{E}-05$ & $3.0311 \mathrm{E}-05$ \\
\hline NI-61 & $1.1380 \mathrm{E}-06$ & $1.1380 \mathrm{E}-06$ & $1.1380 \mathrm{E}-06$ & 4.1379E-06 & $1.4926 \mathrm{E}-06$ & $1.3176 \mathrm{E}-06$ \\
\hline $\mathrm{NI}-62$ & $3.6285 \mathrm{E}-06$ & $3.6285 \mathrm{E}-06$ & $3.6285 \mathrm{E}-06$ & $1.3193 \mathrm{E}-05$ & $4.7590 \mathrm{E}-06$ & $4.2011 \mathrm{E}-06$ \\
\hline NI-64 & $9.2407 \mathrm{E}-07$ & $9.2407 \mathrm{E}-07$ & $9.2407 \mathrm{E}-07$ & $3.3600 \mathrm{E}-06$ & $1.2120 \mathrm{E}-06$ & $1.0699 \mathrm{E}-06$ \\
\hline FE-54 & $9.4643 \mathrm{E}-04$ & $9.4643 \mathrm{E}-04$ & 9.4643E-04 & $3.4413 \mathrm{E}-03$ & $1.2413 \mathrm{E}-03$ & $1.0958 \mathrm{E}-03$ \\
\hline FE-56 & 1.4857E-02 & $1.4857 \mathrm{E}-02$ & $1.4857 \mathrm{E}-02$ & $5.4021 \mathrm{E}-02$ & $1.9486 \mathrm{E}-02$ & $1.7201 \mathrm{E}-02$ \\
\hline FE-57 & $3.4311 \mathrm{E}-04$ & $3.4311 \mathrm{E}-04$ & $3.4311 \mathrm{E}-04$ & $1.2476 \mathrm{E}-03$ & $4.5001 \mathrm{E}-04$ & $3.9726 \mathrm{E}-04$ \\
\hline FE-58 & 4.5662E-05 & $4.5662 \mathrm{E}-05$ & 4.5662E-05 & 1.6603E-04 & $5.9889 \mathrm{E}-05$ & 5.2867E-05 \\
\hline $\mathrm{MO}-92$ & $8.1308 \mathrm{E}-05$ & $8.1686 \mathrm{E}-05$ & $8.1686 \mathrm{E}-05$ & $6.1129 \mathrm{E}-05$ & $2.2050 \mathrm{E}-05$ & $1.9465 \mathrm{E}-05$ \\
\hline MO-94 & $5.0810 \mathrm{E}-05$ & 5.1047E-05 & 5.1047E-05 & $3.8200 \mathrm{E}-05$ & 1.3779E-05 & $1.2164 \mathrm{E}-05$ \\
\hline MO-95 & $8.7528 \mathrm{E}-05$ & 8.7935E-05 & 8.7935E-05 & $6.5805 \mathrm{E}-05$ & 2.3737E-05 & $2.0954 \mathrm{E}-05$ \\
\hline MO-96 & $9.1822 \mathrm{E}-05$ & $9.2249 \mathrm{E}-05$ & $9.2249 \mathrm{E}-05$ & $6.9034 \mathrm{E}-05$ & $2.4901 \mathrm{E}-05$ & 2.1982E-05 \\
\hline MO-97 & $5.2627 \mathrm{E}-05$ & $5.2872 \mathrm{E}-05$ & $5.2872 \mathrm{E}-05$ & $3.9566 \mathrm{E}-05$ & $1.4272 \mathrm{E}-05$ & $1.2599 \mathrm{E}-05$ \\
\hline MO-98 & 1.3316E-04 & $1.3378 \mathrm{E}-04$ & $1.3378 \mathrm{E}-04$ & $1.0012 \mathrm{E}-04$ & $3.6113 \mathrm{E}-05$ & $3.1879 \mathrm{E}-05$ \\
\hline MO-100 & $5.3233 \mathrm{E}-05$ & $5.3480 \mathrm{E}-05$ & $5.3480 \mathrm{E}-05$ & $4.0021 \mathrm{E}-05$ & $1.4436 \mathrm{E}-05$ & $1.2744 \mathrm{E}-05$ \\
\hline MN-55 & $1.0666 \mathrm{E}-04$ & $1.0666 \mathrm{E}-04$ & $1.0666 \mathrm{E}-04$ & $3.8782 \mathrm{E}-04$ & $1.3989 \mathrm{E}-04$ & $1.2349 \mathrm{E}-04$ \\
\hline NA-23 & $7.1453 \mathrm{E}-03$ & $7.1453 \mathrm{E}-03$ & $7.1453 \mathrm{E}-03$ & $3.4976 \mathrm{E}-03$ & $3.4976 \mathrm{E}-03$ & $8.1722 \mathrm{E}-03$ \\
\hline ZR-90 & $1.6788 \mathrm{E}-03$ & $1.6788 \mathrm{E}-03$ & $1.6788 \mathrm{E}-03$ & & & \\
\hline ZR-91 & 3.6610E-04 & 3.6610E-04 & 3.6610E-04 & & & \\
\hline ZR-92 & $5.5959 \mathrm{E}-04$ & $5.5959 \mathrm{E}-04$ & $5.5959 \mathrm{E}-04$ & & & \\
\hline ZR-94 & $5.6709 \mathrm{E}-04$ & $5.6709 \mathrm{E}-04$ & $5.6709 \mathrm{E}-04$ & & & \\
\hline ZR-96 & $9.1361 \mathrm{E}-05$ & $9.1361 \mathrm{E}-05$ & $9.1361 \mathrm{E}-05$ & & & \\
\hline U-235 & $1.2000 \mathrm{E}-03$ & 2.0000E-03 & $2.0000 \mathrm{E}-05$ & & & \\
\hline U-238 & 7.4400E-03 & 7.4400E-03 & 7.4400E-03 & & & \\
\hline B-10 & & & & & $8.1942 \mathrm{E}-03$ & 5.9987E-03 \\
\hline B-11 & & & & & $3.2983 \mathrm{E}-02$ & $2.4145 \mathrm{E}-02$ \\
\hline$C-12$ & & & & & $1.9432 \mathrm{E}-03$ & $1.4225 \mathrm{E}-03$ \\
\hline
\end{tabular}

With regard to the solution itself, we are interested in the keff of the system along with the spatial scalar flux solution and power prediction. For the spatial distribution information, the MCNP tally operations are difficult and it is sufficient to look at a subset of the possible data. In this regard we choose $10 \mathrm{~cm}$ x $10 \mathrm{~cm}$ meshes that run along the lower reflected border 
as shown in Figure 4.1 which amounts to 18 flux tallies. For the energy boundaries, we setup the MCNP tallies to exactly match those of DIF3D. Even after running a billion particle histories in MCNP, we still have errors in the flux tallies in the shielding region of $5 \%$ and some tallies over energy have no contributing particles. As a consequence, one should be careful when considering the MCNP solution a true reference at all points in space with regard to the power prediction and for certain the flux solution.

For our first test problem, the control rod indicated in Figure 4.1 is replaced with outer core material. The eigenvalue summary for this problem is given in Table 4.4. As can be seen, the 1042 group $\mathrm{MC}^{2}-3$ is $54 \mathrm{pcm}$ above the MCNP solution while the standard DIF3DVARIANT transport model is $62 \mathrm{pcm}$ below the MCNP solution. Both DIF3D-FD and DIF3D-VARIANT diffusion solutions are nearly identical to the DIF3D transport result. Using the improved region cross section data in DIF3D, one can see that the DIF3D transport calculation changes by almost $100 \mathrm{pcm}$ to nearly match the TWODANT result obtained directly from $\mathrm{MC}^{2}-3$ (4 pcm difference). These results can generally be considered excellent given the vast reduction in computational effort in running DIF3D versus MCNP. Of course a comparison of the fluxes and power is the more important aspect of the GAMSOR analysis.

Table 4.4. Configuration 1 keff Results

\begin{tabular}{|l|c|c|c|}
\hline \multicolumn{1}{|c|}{ Problem } & Group & $\mathrm{k}_{\mathrm{eff}}$ & $\begin{array}{c}\Delta \mathrm{k} \text { from MCNP } \\
(\mathrm{pcm})\end{array}$ \\
\hline DIF3D-FD & 33 & 1.22610 & -66 \\
\hline DIF3D-VARIANT diffusion & 33 & 1.22607 & -69 \\
\hline DIF3D-VARIANT P7-P5 & 33 & 1.22614 & -62 \\
\hline TWODANT + MC 2 -3 & 1042 & 1.22730 & 54 \\
\hline $\begin{array}{l}\text { TWODANT+MC }-3 \\
\rightarrow \text { DIF3D-VARIANT }\end{array}$ & 33 & 1.22726 & 50 \\
\hline \multicolumn{1}{|c|}{ MCNP } & $1.22676 \pm 0.00001$ & \\
\hline
\end{tabular}

As discussed, we have chosen 18 tallies for comparing the MCNP and DIF3D codes shown earlier in Figure 4.1. To begin the comparison, we show the direct comparison of neutron fluxes for tallies 1, 6, and 10 in Figure 4.3 where we neglect the MCNP statistical errors to make the graphs readable. The DIF3D-VARIANT P7-P5 solution is used for all figures in this section. The flux solution at each tally was normalized to have the same area under the curve where the flux magnitude values are shown on the left hand side and the scale for the errors between the MCNP and deterministic solutions are given on the right. As can be seen, the errors near the peak of the flux are relatively low and generally below $1 \%$. As the flux magnitude drops below $1 / 10^{\text {th }}$ of the peak, the error is generally seen to increase to far greater than $10 \%$. This increase in error corresponds well with the error bars on the MCNP results which we tabulated for the first tally position in Table 4.5.

From Table 4.5, below about $200 \mathrm{eV}$ one can see that the MCNP solutions are unusable at the core center even though we have executed 1 billion particles. We clearly need to use variance reduction to improve the solution in these lower energy groups. At the upper most energy group, the MCNP uncertainty is $\sim 1 \%$, which indicates that the DIF3D solution with an error of $\sim 3 \%$ might not be too unreasonable. Overall, we are more interested in the power heating as that is the function of GAMSOR, but it is should be clear that if the flux 
solution has significant error in it, it is possible to get rather substantial errors in the power heating.

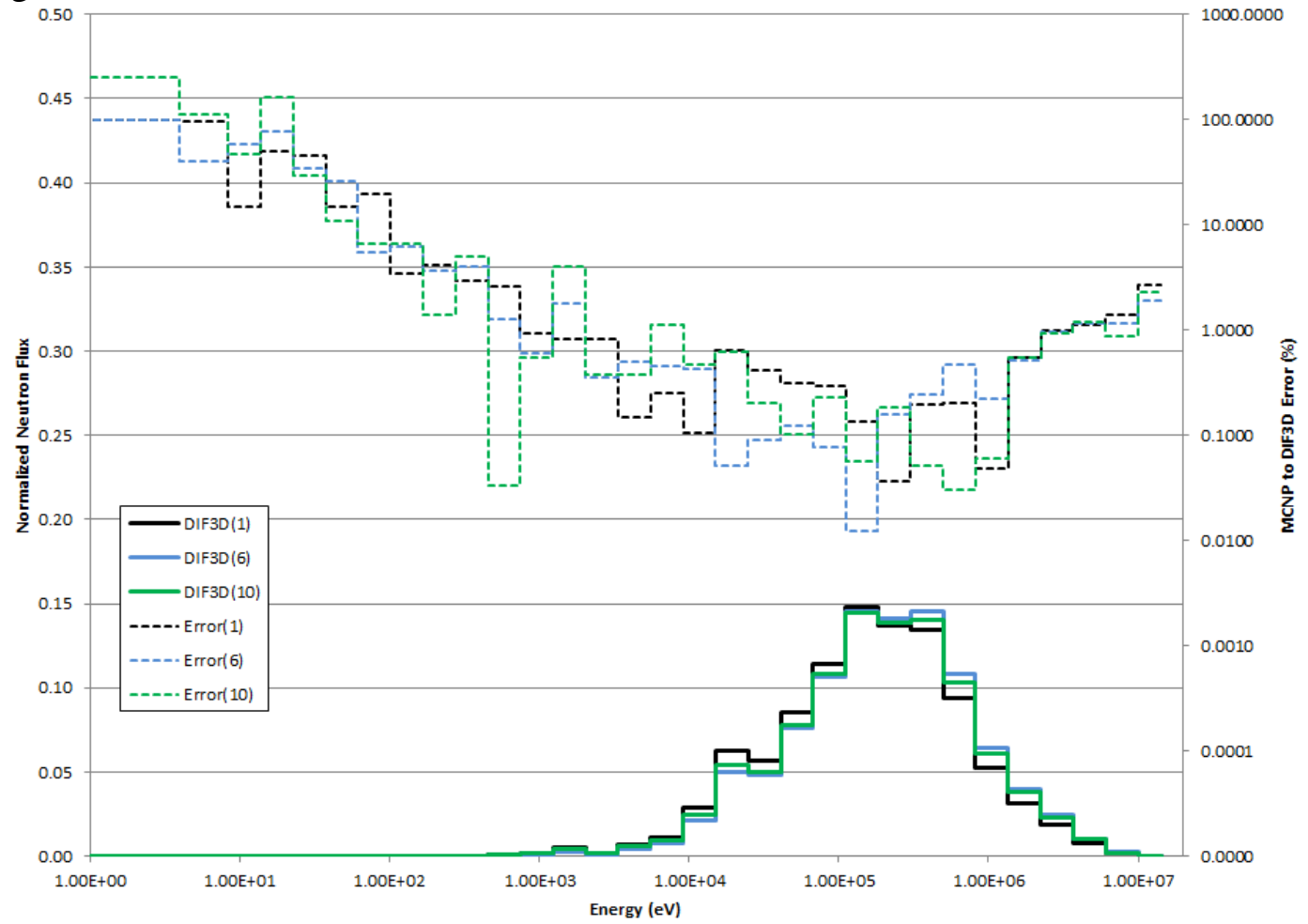

Figure 4.3. Configuration 1 Neutron Flux Spectrum Results for Tallies 1, 6, and 10.

Table 4.5. Configuration 1 MCNP Neutron Flux and Statistical Errors for the $1^{\text {st }}$ Tally

\begin{tabular}{|c|c|c|c|c|c|}
\hline Energy(eV) & \multicolumn{2}{|c|}{ Neutron Flux } & Energy(eV) & \multicolumn{2}{c|}{ Neutron Flux } \\
\hline $1.42 \mathrm{E}+07$ & $9.52 \mathrm{E}-08$ & $0.85 \%$ & $3.35 \mathrm{E}+03$ & $2.11 \mathrm{E}-06$ & $0.10 \%$ \\
\hline $1.00 \mathrm{E}+07$ & $1.74 \mathrm{E}-06$ & $0.20 \%$ & $2.03 \mathrm{E}+03$ & $5.53 \mathrm{E}-06$ & $0.10 \%$ \\
\hline $6.07 \mathrm{E}+06$ & $8.30 \mathrm{E}-06$ & $0.10 \%$ & $1.23 \mathrm{E}+03$ & $2.20 \mathrm{E}-06$ & $0.16 \%$ \\
\hline $3.68 \mathrm{E}+06$ & $1.96 \mathrm{E}-05$ & $0.06 \%$ & $7.49 \mathrm{E}+02$ & $5.85 \mathrm{E}-07$ & $0.30 \%$ \\
\hline $2.23 \mathrm{E}+06$ & $3.32 \mathrm{E}-05$ & $0.05 \%$ & $4.54 \mathrm{E}+02$ & $1.20 \mathrm{E}-07$ & $0.65 \%$ \\
\hline $1.35 \mathrm{E}+06$ & $5.62 \mathrm{E}-05$ & $0.04 \%$ & $2.75 \mathrm{E}+02$ & $2.52 \mathrm{E}-08$ & $1.35 \%$ \\
\hline $8.21 \mathrm{E}+05$ & $1.00 \mathrm{E}-04$ & $0.04 \%$ & $1.67 \mathrm{E}+02$ & $3.70 \mathrm{E}-09$ & $3.59 \%$ \\
\hline $4.98 \mathrm{E}+05$ & $1.43 \mathrm{E}-04$ & $0.03 \%$ & $1.01 \mathrm{E}+02$ & $6.84 \mathrm{E}-10$ & $9.66 \%$ \\
\hline $3.02 \mathrm{E}+05$ & $1.46 \mathrm{E}-04$ & $0.03 \%$ & $6.14 \mathrm{E}+01$ & $1.28 \mathrm{E}-10$ & $18.65 \%$ \\
\hline $1.83 \mathrm{E}+05$ & $1.58 \mathrm{E}-04$ & $0.03 \%$ & $3.73 \mathrm{E}+01$ & $1.94 \mathrm{E}-11$ & $36.99 \%$ \\
\hline $1.11 \mathrm{E}+05$ & $1.23 \mathrm{E}-04$ & $0.03 \%$ & $2.26 \mathrm{E}+01$ & $9.11 \mathrm{E}-12$ & $52.45 \%$ \\
\hline $6.74 \mathrm{E}+04$ & $9.13 \mathrm{E}-05$ & $0.03 \%$ & $1.37 \mathrm{E}+01$ & $1.19 \mathrm{E}-11$ & $61.15 \%$ \\
\hline $4.09 \mathrm{E}+04$ & $6.07 \mathrm{E}-05$ & $0.03 \%$ & $8.32 \mathrm{E}+00$ & $2.64 \mathrm{E}-13$ & $100.00 \%$ \\
\hline $2.48 \mathrm{E}+04$ & $6.75 \mathrm{E}-05$ & $0.03 \%$ & $3.93 \mathrm{E}+00$ & $3.33 \mathrm{E}-11$ & $65.97 \%$ \\
\hline $1.50 \mathrm{E}+04$ & $3.06 \mathrm{E}-05$ & $0.05 \%$ & $5.32 \mathrm{E}-01$ & $0.00 \mathrm{E}+00$ & $0.00 \%$ \\
\hline $9.12 \mathrm{E}+03$ & $1.17 \mathrm{E}-05$ & $0.06 \%$ & $4.17 \mathrm{E}-01$ & $0.00 \mathrm{E}+00$ & $0.00 \%$ \\
\hline $5.53 \mathrm{E}+03$ & $7.46 \mathrm{E}-06$ & $0.08 \%$ & & & \\
\hline
\end{tabular}


Continuing, the direct comparison of neutron fluxes at tallies 12, 14, and 17 are given in Figure 4.4. Compared with Figure 4.3, we see a considerable increase in the error between DIF3D and MCNP for the blanket, reflector, and shield positions. For a bulk of the energy range, the errors are well above 5\% and in the lower energy regime the errors are effectively $100 \%$. Similar to the core region results, we neglected the MCNP error bars which we present for the $17^{\text {th }}$ tally region in Table 4.6. As can be seen, the DIF3D errors for the lower energies are normal as the MCNP code does not obtain a solution. While we did consider running more than 1 billion particle histories, it is rather unrealistic to assume that running 9 billion more will result in non-zero tallies in these energy groups without some measure of variance reduction. With regard to their contribution to the heating, given the near zero flux magnitude, we can safely assume that they will only impact the heating in a minor way.

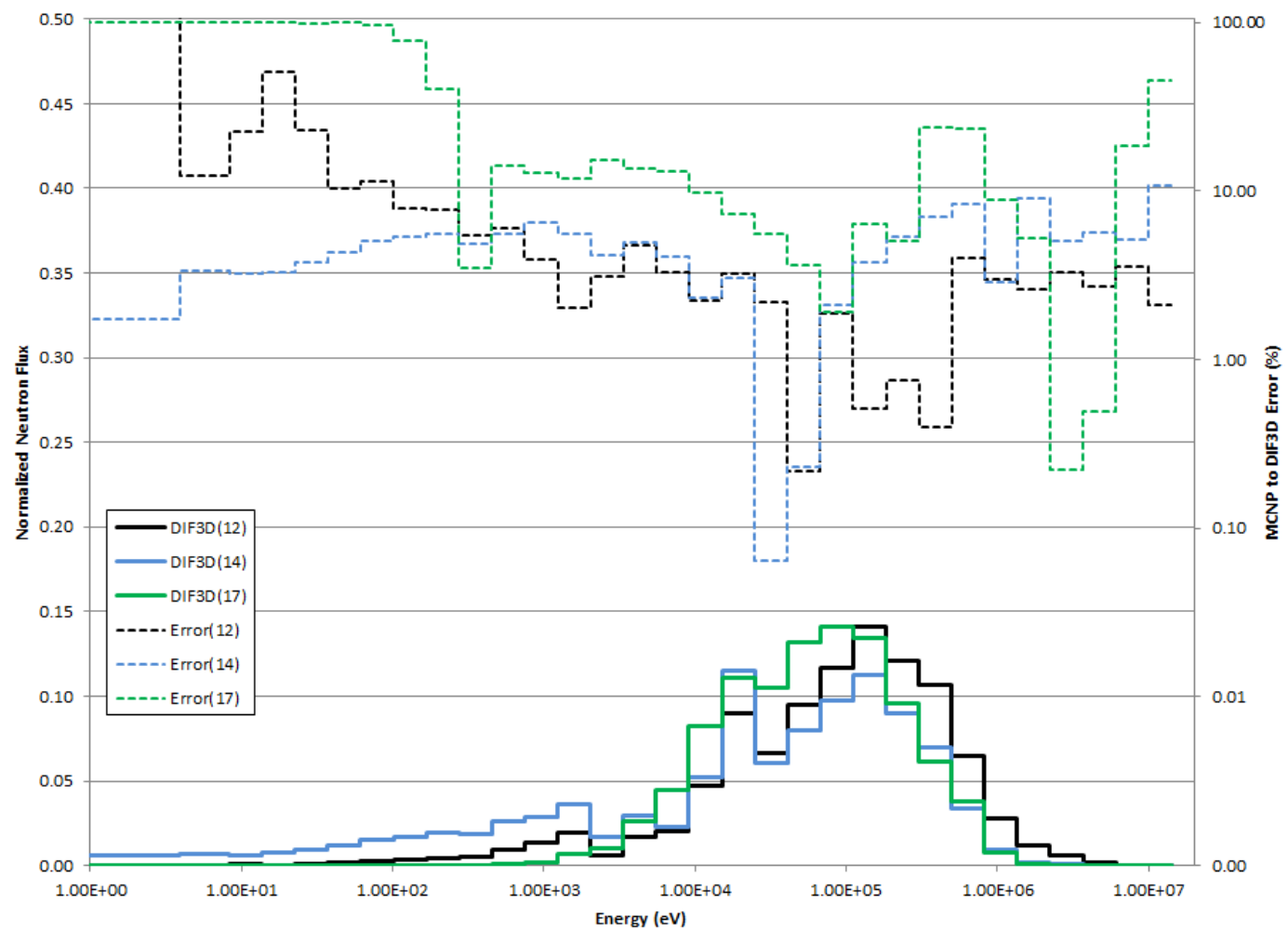

Figure 4.4. Configuration 1 Neutron Flux Spectrum Results for Tallies 12, 14, and 17.

Figure 4.5 and Figure 4.6 show the gamma flux spectrum distributions for the same tally points used for the neutron flux results presented above. A quick look the gamma flux results errors and one finds they are generally above $5 \%$ at all spatial locations where we note the MCNP statistical errors are less than 1\%. This indicates that there is a systematic bias in the gamma flux calculation which is most likely due to the gamma cross section data treatment. It is interesting to note that the neutron tallies had numerous energy groups that had zero neutrons in it while the gamma energy tallies all had non-zero tallies with a maximum error of 75\% error with the highest energy tally in the outer shielding region. In fact, almost none of the other tally positions in any energy group had reported errors above 5\%. This is of course easily explainable by the differences in energy boundaries chosen for the neutron and gamma particles where the neutron energy boundaries cover the lower energy regime in a considerably finer fashion. 
Table 4.6. Configuration 1 MCNP Neutron Flux and Statistical Errors for the $17^{\text {th }}$ Tally

\begin{tabular}{|r|r|r|c|r|r|}
\hline Energy(eV) & \multicolumn{2}{|c|}{ Neutron Flux } & Energy(eV) & \multicolumn{2}{c|}{ Neutron Flux } \\
\hline $1.42 \mathrm{E}+07$ & $1.83 \mathrm{E}-11$ & $41.32 \%$ & $3.35 \mathrm{E}+03$ & $6.83 \mathrm{E}-08$ & $0.5 \%$ \\
\hline $1.00 \mathrm{E}+07$ & $1.08 \mathrm{E}-10$ & $18.05 \%$ & $2.03 \mathrm{E}+03$ & $4.48 \mathrm{E}-08$ & $0.7 \%$ \\
\hline $6.07 \mathrm{E}+06$ & $4.98 \mathrm{E}-10$ & $10.00 \%$ & $1.23 \mathrm{E}+03$ & $1.37 \mathrm{E}-08$ & $1.1 \%$ \\
\hline $3.68 \mathrm{E}+06$ & $1.72 \mathrm{E}-09$ & $5.54 \%$ & $7.49 \mathrm{E}+02$ & $3.63 \mathrm{E}-09$ & $2.0 \%$ \\
\hline $2.23 \mathrm{E}+06$ & $6.89 \mathrm{E}-09$ & $2.74 \%$ & $4.54 \mathrm{E}+02$ & $6.76 \mathrm{E}-10$ & $4.2 \%$ \\
\hline $1.35 \mathrm{E}+06$ & $4.93 \mathrm{E}-08$ & $1.06 \%$ & $2.75 \mathrm{E}+02$ & $1.17 \mathrm{E}-10$ & $9.2 \%$ \\
\hline $8.21 \mathrm{E}+05$ & $1.70 \mathrm{E}-07$ & $0.57 \%$ & $1.67 \mathrm{E}+02$ & $2.06 \mathrm{E}-11$ & $21.2 \%$ \\
\hline $4.98 \mathrm{E}+05$ & $2.75 \mathrm{E}-07$ & $0.40 \%$ & $1.01 \mathrm{E}+02$ & $2.94 \mathrm{E}-12$ & $39.7 \%$ \\
\hline $3.02 \mathrm{E}+05$ & $5.33 \mathrm{E}-07$ & $0.28 \%$ & $6.14 \mathrm{E}+01$ & $0.00 \mathrm{E}+00$ & $0.0 \%$ \\
\hline $1.83 \mathrm{E}+05$ & $7.40 \mathrm{E}-07$ & $0.22 \%$ & $3.73 \mathrm{E}+01$ & $2.04 \mathrm{E}-13$ & $100.0 \%$ \\
\hline $1.11 \mathrm{E}+05$ & $8.13 \mathrm{E}-07$ & $0.20 \%$ & $2.26 \mathrm{E}+01$ & $0.00 \mathrm{E}+00$ & $0.0 \%$ \\
\hline $6.74 \mathrm{E}+04$ & $8.05 \mathrm{E}-07$ & $0.19 \%$ & $1.37 \mathrm{E}+01$ & $0.00 \mathrm{E}+00$ & $0.0 \%$ \\
\hline $4.09 \mathrm{E}+04$ & $6.52 \mathrm{E}-07$ & $0.20 \%$ & $8.32 \mathrm{E}+00$ & $0.00 \mathrm{E}+00$ & $0.0 \%$ \\
\hline $2.48 \mathrm{E}+04$ & $7.00 \mathrm{E}-07$ & $0.20 \%$ & $3.93 \mathrm{E}+00$ & $0.00 \mathrm{E}+00$ & $0.0 \%$ \\
\hline $1.50 \mathrm{E}+04$ & $5.34 \mathrm{E}-07$ & $0.22 \%$ & $5.32 \mathrm{E}-01$ & $0.00 \mathrm{E}+00$ & $0.0 \%$ \\
\hline $9.12 \mathrm{E}+03$ & $2.98 \mathrm{E}-07$ & $0.27 \%$ & $4.17 \mathrm{E}-01$ & $0.00 \mathrm{E}+00$ & $0.0 \%$ \\
\hline $5.53 \mathrm{E}+03$ & $1.77 \mathrm{E}-07$ & $0.35 \%$ & & & \\
\hline
\end{tabular}

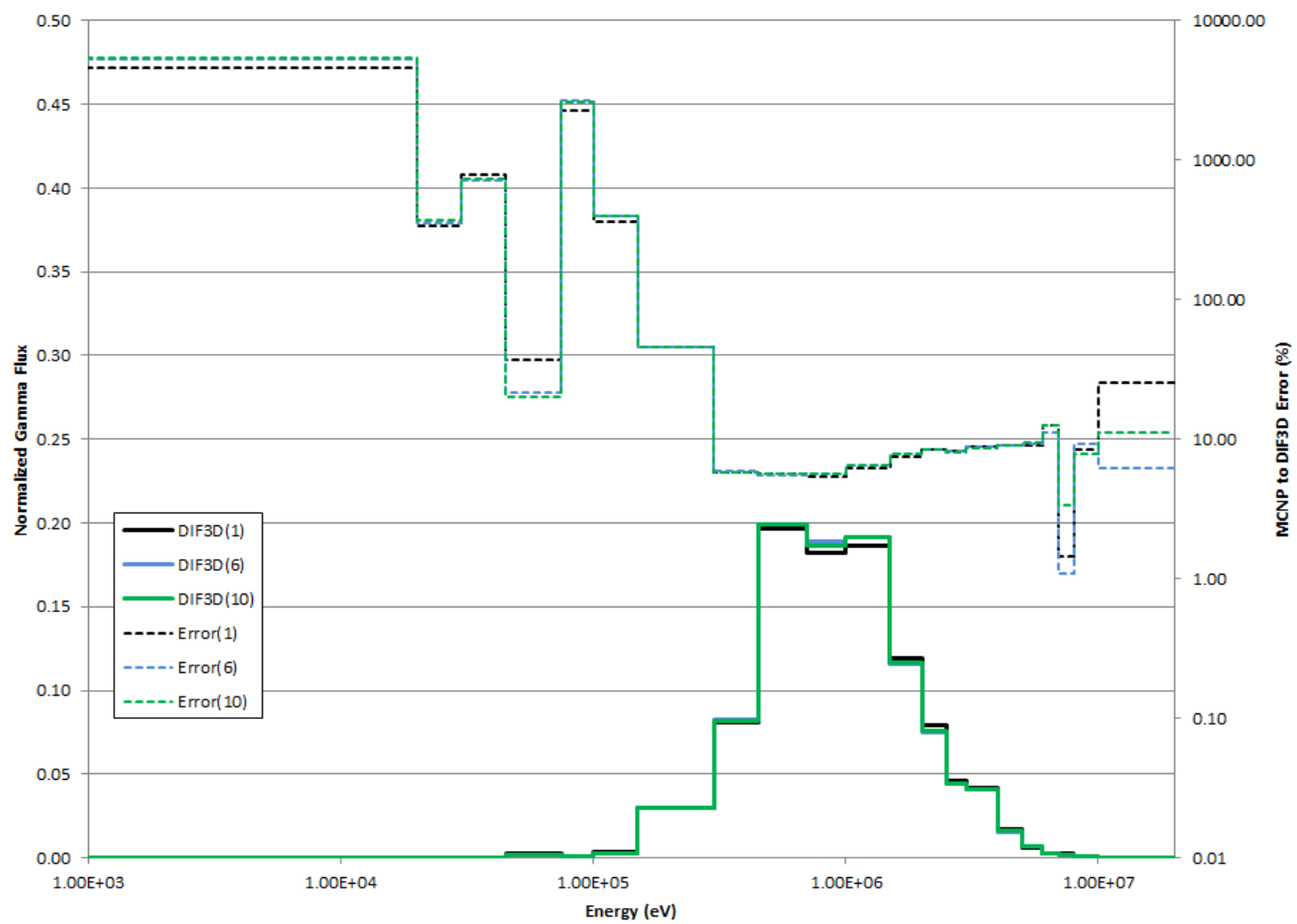

Figure 4.5. Configuration 1 Gamma Flux Spectrum Results for Tallies 1, 6, and 10. 
Comparing Figure 4.5 with Figure 4.6, one can see a notable and dramatic increase in the error in the shielding region. While there is a significant error in the blanket and reflector when compared to the core regions, the shielding region errors indicate that the DIF3D solution is completely erroneous. Of the three tallies in the shielding region (meshes 16, 17, and 18), the 16th tally region has the least error and both the 17th and 18th have errors greater than $50 \%$ at all points. Looking at the compositions in Table 4.3, it does not go without noting that this is the only material with B-10, B-11, and C-12 included. Because this is the first of three such calculations on this geometry, we defer any conclusions until after discussing the other two configurations.

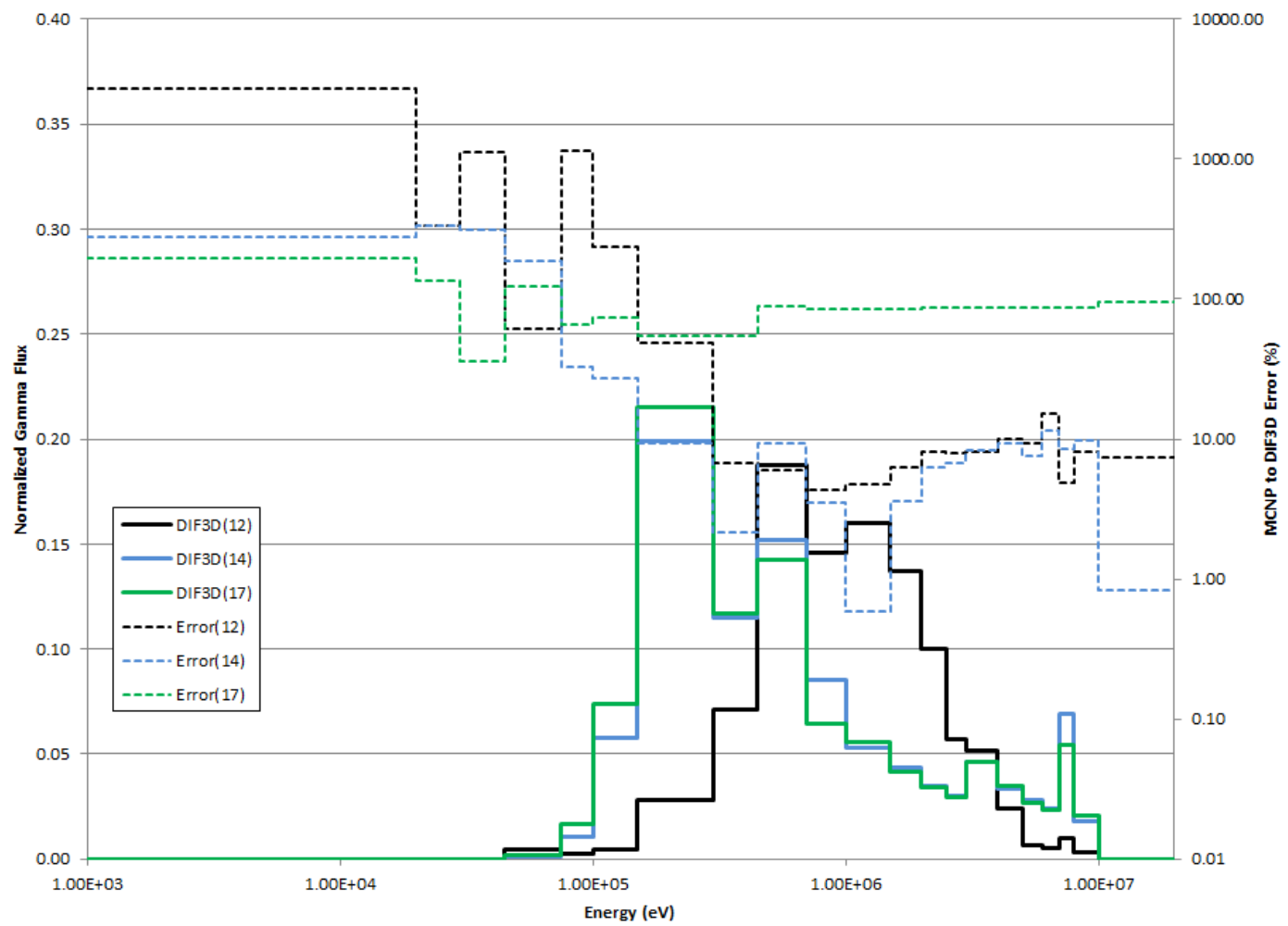

Figure 4.6. Configuration 1 Gamma Flux Spectrum Results for Tallies 12, 14, and 17.

As mentioned, the most important part to compare is the power density contributions from both neutrons and gammas. Figure 4.7 shows the MCNP and DIF3D transport power heating solutions plotted together with the 68\% confidence intervals error bars included on the MCNP solution. The reported MCNP error bars are so low that they are virtually non-existent in the graph. The error between the DIF3D and MCNP solution is plotted on the right hand scale which indicates a less than 13\% error for most of the tallies except for the shielding regions all of which have greater than 100\% error. Looking at the MCNP and DIF3D solutions, it is clear that the DIF3D solution is predicting a power deposition rate that is below that of MCNP for all three positions in the shielding. Besides this obvious error, it is interesting to note that the core power distribution is within $3 \%$ of each other while the blanket error has the second largest errors of $\sim 13 \%$. This is a bit counter intuitive as the core and blanket regions effectively have the same compositions except for the U-235 content. 


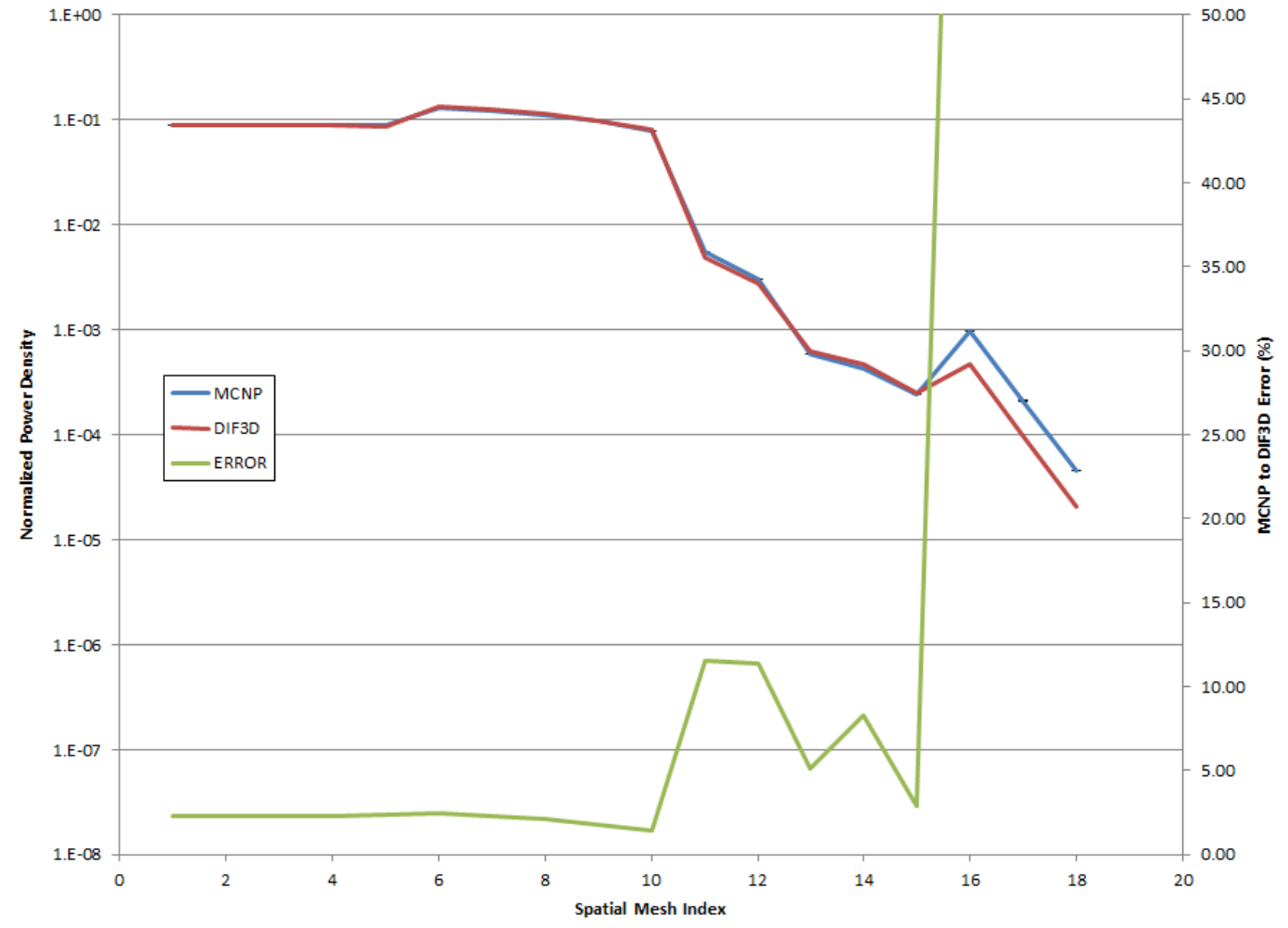

Figure 4.7. Neutron and Gamma Heating Results for Configuration 1.

To shed more light on this aspect, we collected the power heating results from each approach taken in Table 4.4, except the TWODANT solution, and present the errors in Figure 4.8. As can be seen, the DIF3D-FD solution is the only one that is not fully consistent with the others. This is primarily a mesh refinement issue that does not impact the DIF3DVARIANT approach as much because of its high order spatial approximation. Applying more spatial mesh refinement in DIF3D-FD will reduce the errors to match those seen in the diffusion version of DIF3D-VARIANT.

Also included in Figure 4.8 is a "FIXSRC" diffusion case of DIF3D-VARIANT. In our earlier discussion, we noted how important it was to model the correct polynomial distribution in DIF3D-VARIANT. This calculation shows the error that results when switching the diffusion calculation to use the flattened gamma source over a $10 \times 10 \mathrm{~cm}^{2}$ mesh region. As can be seen, the impact is relatively minor on this particular problem but significant enough to validate the correct distribution is used.

Looking at all of the results in Figure 4.8, one can state that, except for the shielding region, the various DIF3D-VARIANT solutions consistently produce results that are nearly $10 \%$ error with respect to the MCNP power heating. As a consequence of this set of calculations, the remainder of the configurations is only calculated with the DIF3DVARIANT code using diffusion theory and the same transport options. 


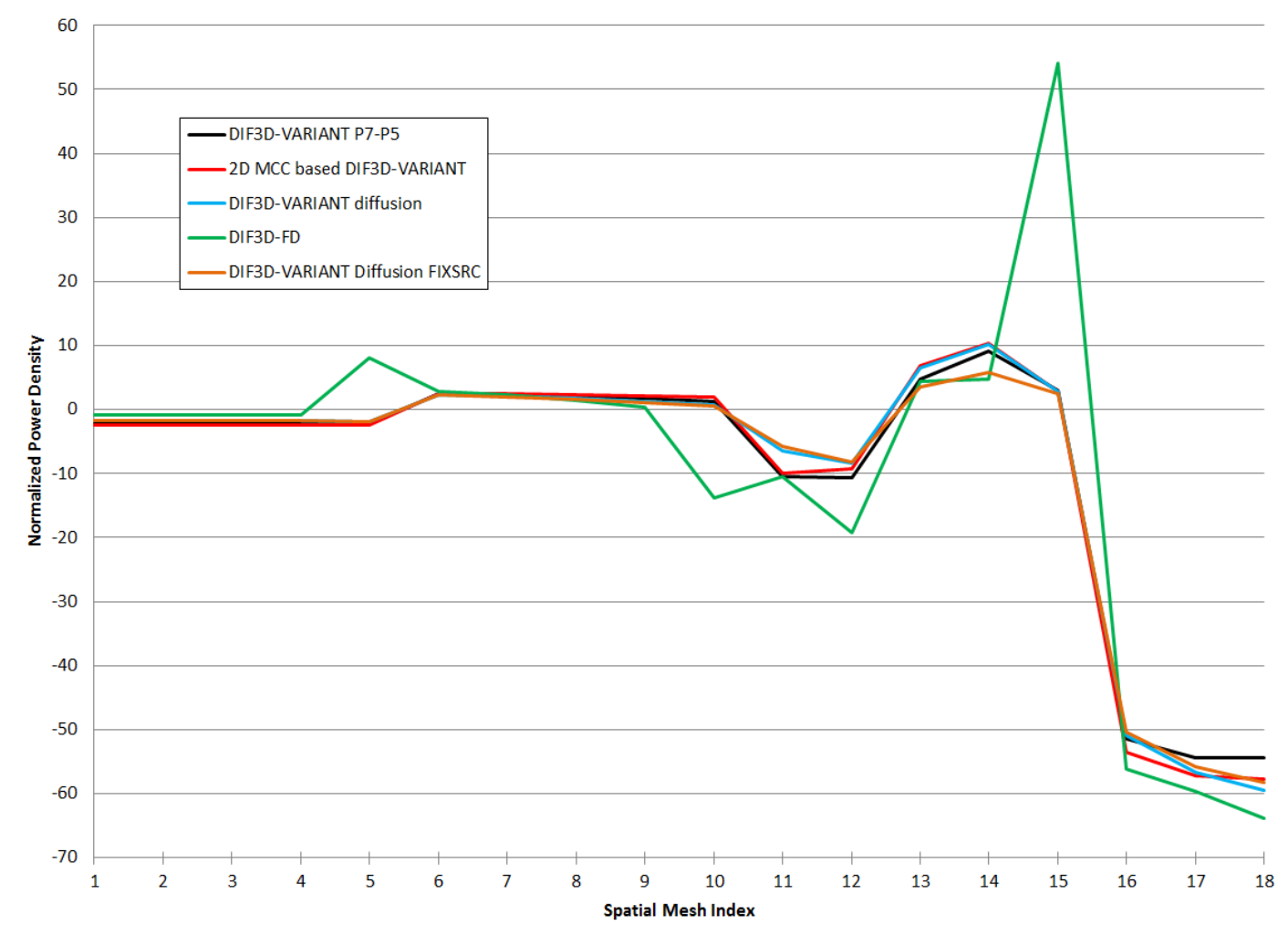

Figure 4.8. Deterministic Errors in Neutron and Gamma Heating Results for Configuration 1.

\subsubsection{Rodded Uranium Dominated Core Problem}

In the second configuration, the control rod shown in Figure 4.1 is filled with the control rod material provided in Table 4.3. The same number of particle histories were ran in MCNP and the same cross section generation approach was taken in $\mathrm{MC}^{2}-3$ along with the above selected transport and diffusion options. Given this, there is no point to inspecting the neutron or gamma fluxes individually like that done for configuration 1 and we instead just summarize the $\mathrm{k}_{\text {eff }}$ results and the power heating results. Table 4.7 lists the $\mathrm{k}_{\mathrm{eff}}$ results for the DIF3D calculations where we note that the TWODANT based approaches were dropped for brevity.

Table 4.7. Configuration 2 keff Results

\begin{tabular}{|c|c|c|c|}
\hline Code & Group & $\mathrm{k}_{\text {eff }}$ & $\begin{array}{c}\Delta \mathrm{k} \text { from MCNP } \\
(\mathrm{pcm})\end{array}$ \\
\hline DIF3D-FD & 33 & 1.20723 & -52 \\
\hline DIF3D-VARIANT diffusion & 33 & 1.20715 & -60 \\
\hline DIF3D-VARIANT P7-P5 & 33 & 1.20755 & -20 \\
\hline \multicolumn{2}{|l|}{ MCNP } & $1.20775 \pm 0.00002$ & \\
\hline
\end{tabular}

In Table 4.7, one can see that the diffusion calculations produce nearly identical eigenvalues which are greater than 50 pcm below the MCNP solution. The DIF3D transport solution shows an improved eigenvalue solution to the diffusion results yielding only $20 \mathrm{pcm}$ below the MCNP one, however, based upon the results in Table 4.4, we can assume this is 
more than likely just cancellation of error given that configuration 2 is more difficult than configuration 1 with regard to the spectrum and multi-group cross section generation.

Figure 4.9 shows the combined neutron and gamma heating predictions from MCNP and DIF3D for the calculations carried out in Table 4.7. The MCNP error bars are not included as the reported values are not visible on this plot. There are clearly two locations where the error between MCNP and DIF3D is large and both again correspond to the presence of B-10, B-11, and C-12. In those two locations, one should note that the DIF3D diffusion theory solutions both yield solutions that have lower error than the transport case.

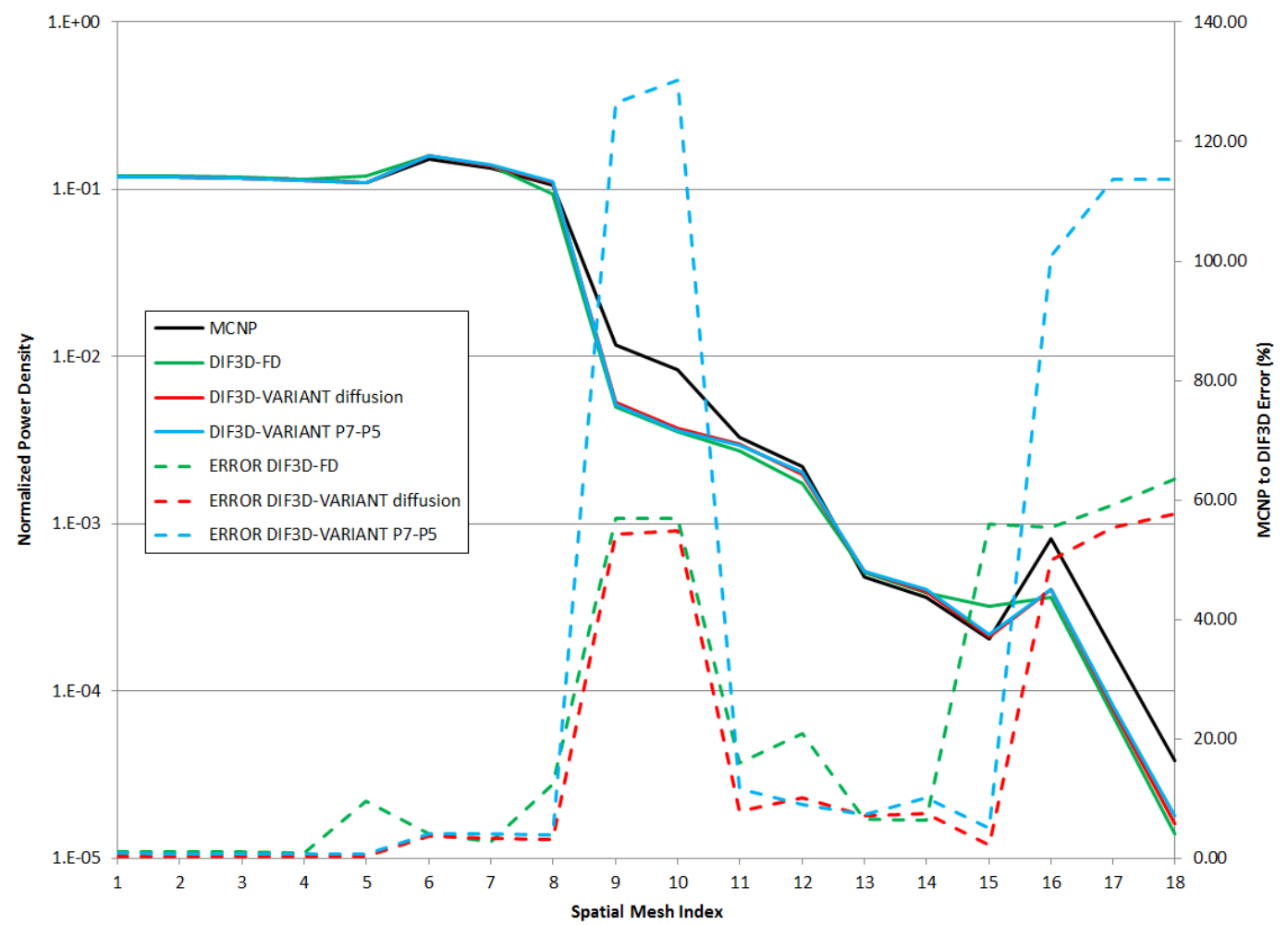

Figure 4.9. Neutron and Gamma Heating Results for Configuration 2.

To explain the differences, we first look at the both the neutron and gamma flux distributions at the first tally, the core center, where the error is smallest and the $9^{\text {th }}$ and $16^{\text {th }}$ tally where the errors are much higher. Figure 4.10 shows the neutron spectrum at each location while Figure 4.11 shows the gamma spectrum at each location.

Starting with the neutron spectrum in Figure 4.10, near the peak of the flux, the errors in the neutron fluxes are relatively small with much less than a few \% error. In the control rod and shielding region, the errors are much higher with about $5 \%$ average error in the control rod and 10\% average error in the shield region. Looking at the gamma spectrum in Figure 4.11, one can see that the average error in the core center is less than $10 \%$ near the peak but for the control rod we have $60 \%$ errors which correspond well with the large errors in the power heating prediction. 


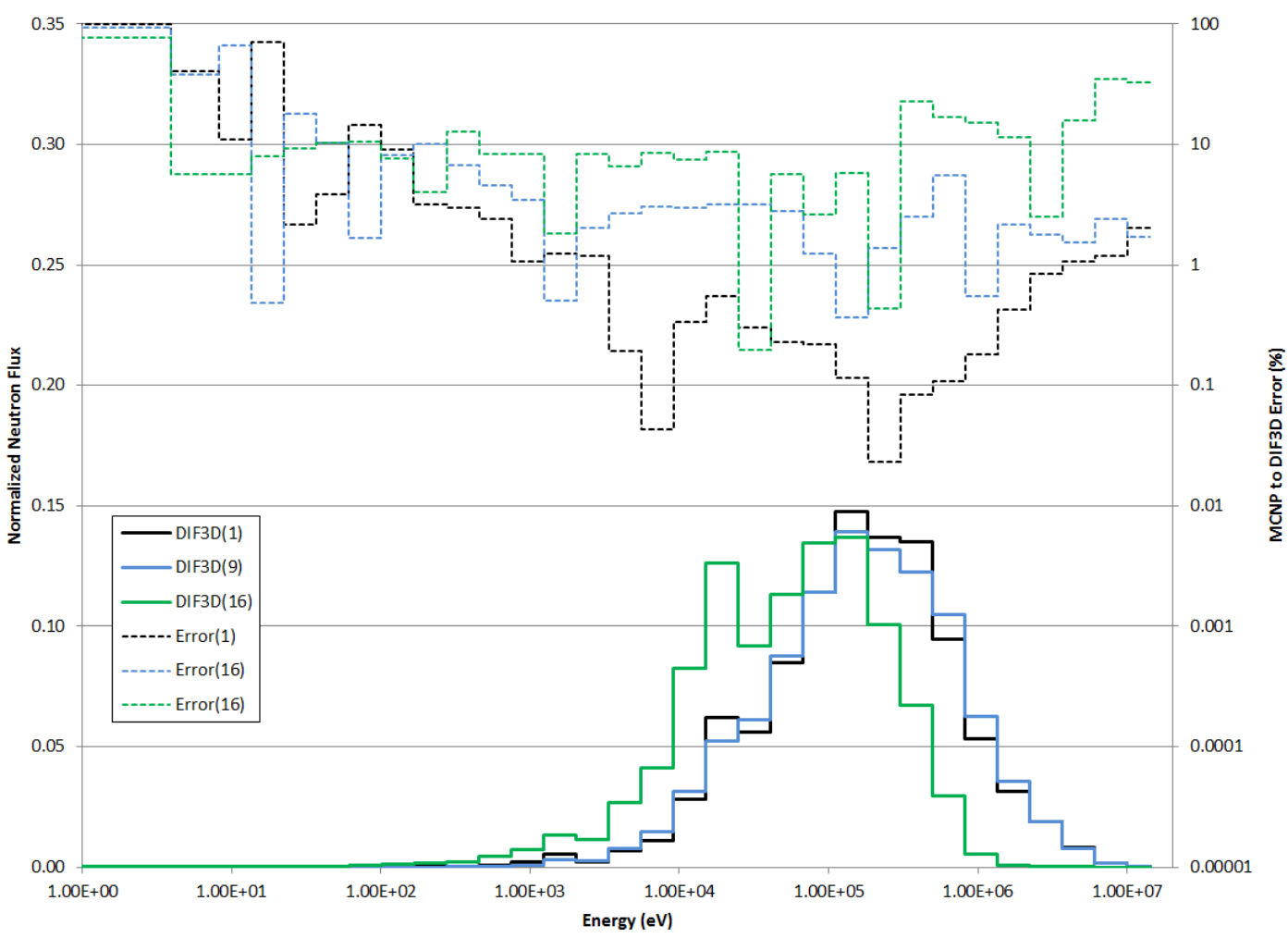

Figure 4.10. Neutron Flux Results for Tallies 1, 9, and 16 of Configuration 2.

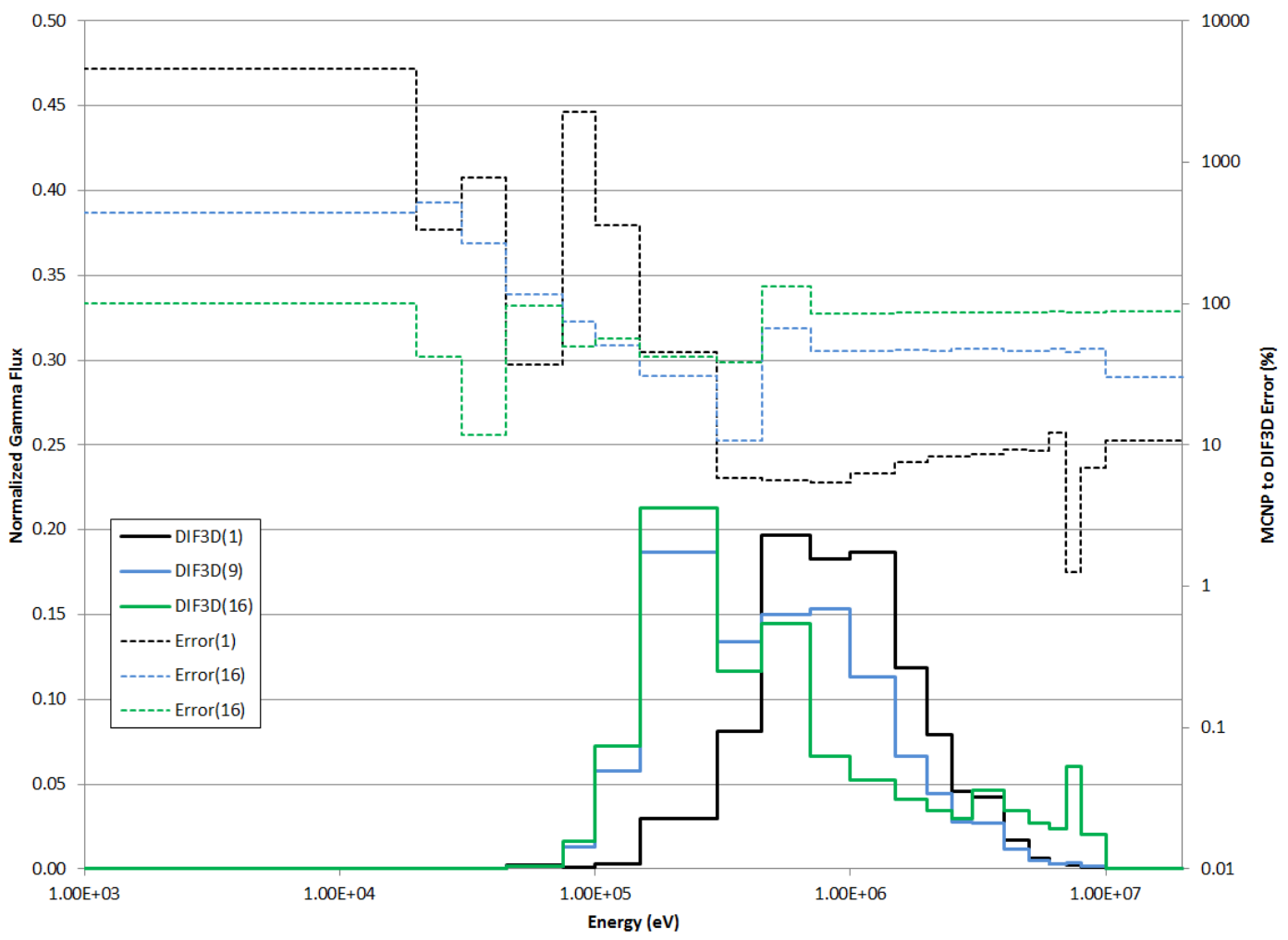

Figure 4.11. Gamma Flux Results for Tallies 1, 9, and 16 of Configuration 2. 


\subsubsection{Rodded Plutonium Dominated Core Problem}

The last configuration we want to test with Figure 4.1 is a plutonium dominated core configuration. The blanket, reflector, shield, and control rod all have the same composition as the previous uranium compositions shown in Table 4.3 thus we only give the two additional inner and outer core compositions in Table 4.8. As can be seen, compared with the previous compositions, we include most of the important actinide isotopes for a typical Pu based system.

Table 4.8. Material Compositions for the Configuration 3 Two-Dimensional Test Problem

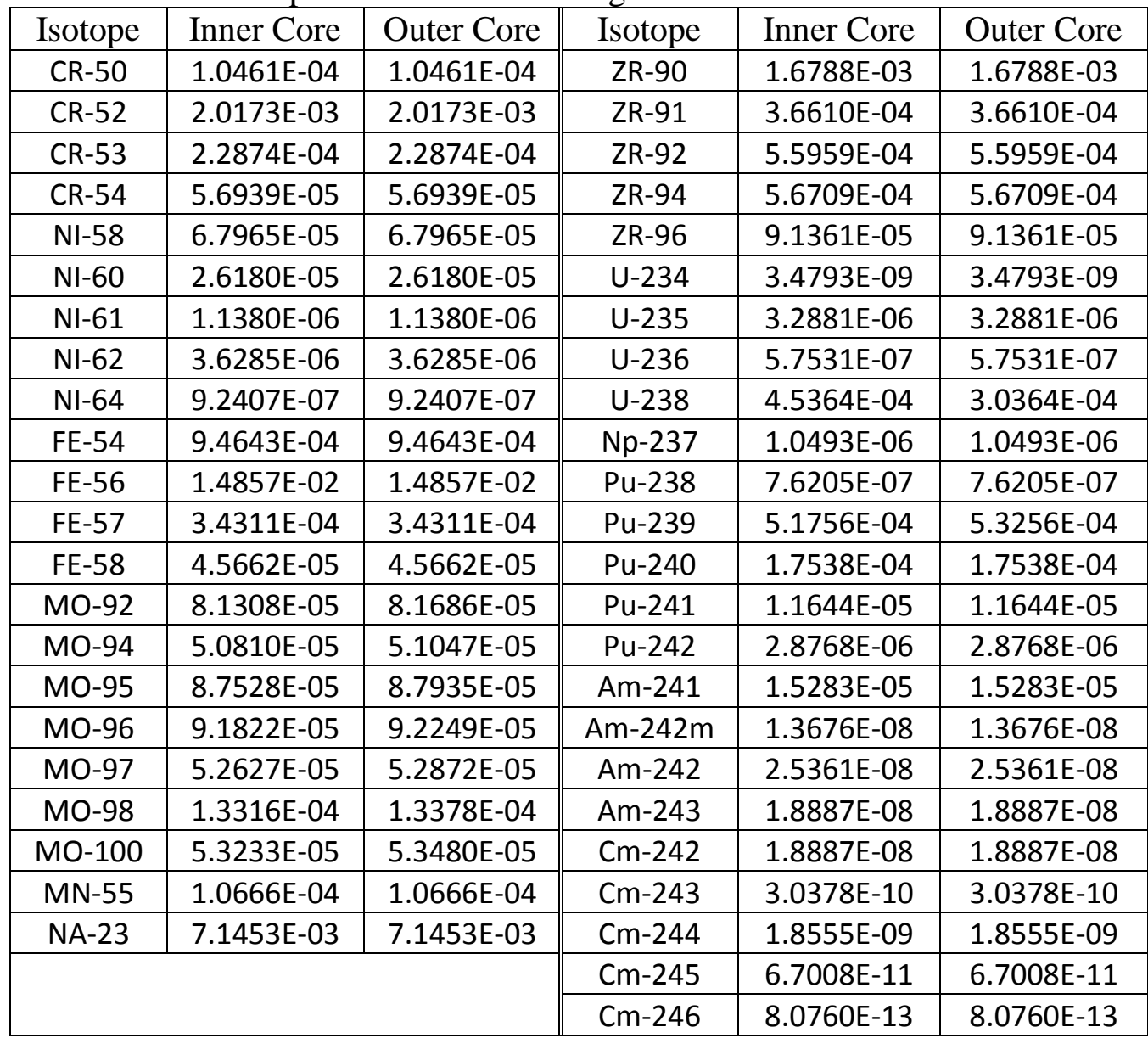

Using the updated compositions, we used the same procedure to generate cross sections with $\mathrm{MC}^{2}-3$ (without TWODANT) for the core problem. To generate a reference, we again ran MCNP with 1 billion particle histories and used the same DIF3D options to obtain the keff results summarized in Table 4.9. As can be seen, the keff errors are considerably larger with over $100 \mathrm{pcm}$ of error between the DIF3D transport result and the MCNP solution. This indicates that the inclusion of other actinides alters the accuracy of the DIF3D approach. Even including the TWODANT calculation in $\mathrm{MC}^{2}-3$ with 1042 groups results in 100 pcm error which indicates that there is a fundamental difference between the cross section handling although it is rather small. 
Table 4.9. Configuration 3 keff Results

\begin{tabular}{|l|c|c|c|}
\hline \multicolumn{1}{|c|}{ Code } & Group & keff & $\begin{array}{c}\Delta \mathrm{k} \text { from MCNP } \\
(\mathrm{pcm})\end{array}$ \\
\hline DIF3D-FD & 33 & 1.12373 & -157 \\
\hline DIF3D-VARIANT diffusion & 33 & 1.12344 & -186 \\
\hline DIF3D-VARIANT P7-P5 & 33 & 1.12406 & -124 \\
\hline TWODANT + MC 2 -3 & 1042 & 1.12423 & -107 \\
\hline \multicolumn{2}{|c|}{ MCNP } & $1.12530 \pm 0.00002$ & \\
\hline
\end{tabular}

The power prediction is given in Figure 4.12, which, when compared against the rodded uranium case of Figure 4.9, shows a considerable change in the power shape. This is of course expected given the earlier power shapes of all three configurations in Figure 4.2. From Figure 4.9, one can observe a substantial under prediction of the power in the control rod and shielding regions which was not the case in the previous two configuration problems. The control rod and shielding errors are less than $10 \%$ but the blanket and reflector errors are well over 30\% error with DIF3D consistently over predicting the solution. The DIF3D-FD result is the worst, with a peak error above $120 \%$ whereas the DIF3D-VARIANT diffusion solution maxes out at $110 \%$ error. The difference between these two calculations is a result of additional spatial mesh refinement needed in DIF3D-FD. Using transport the peak error drops to $50 \%$ in the radial reflector.

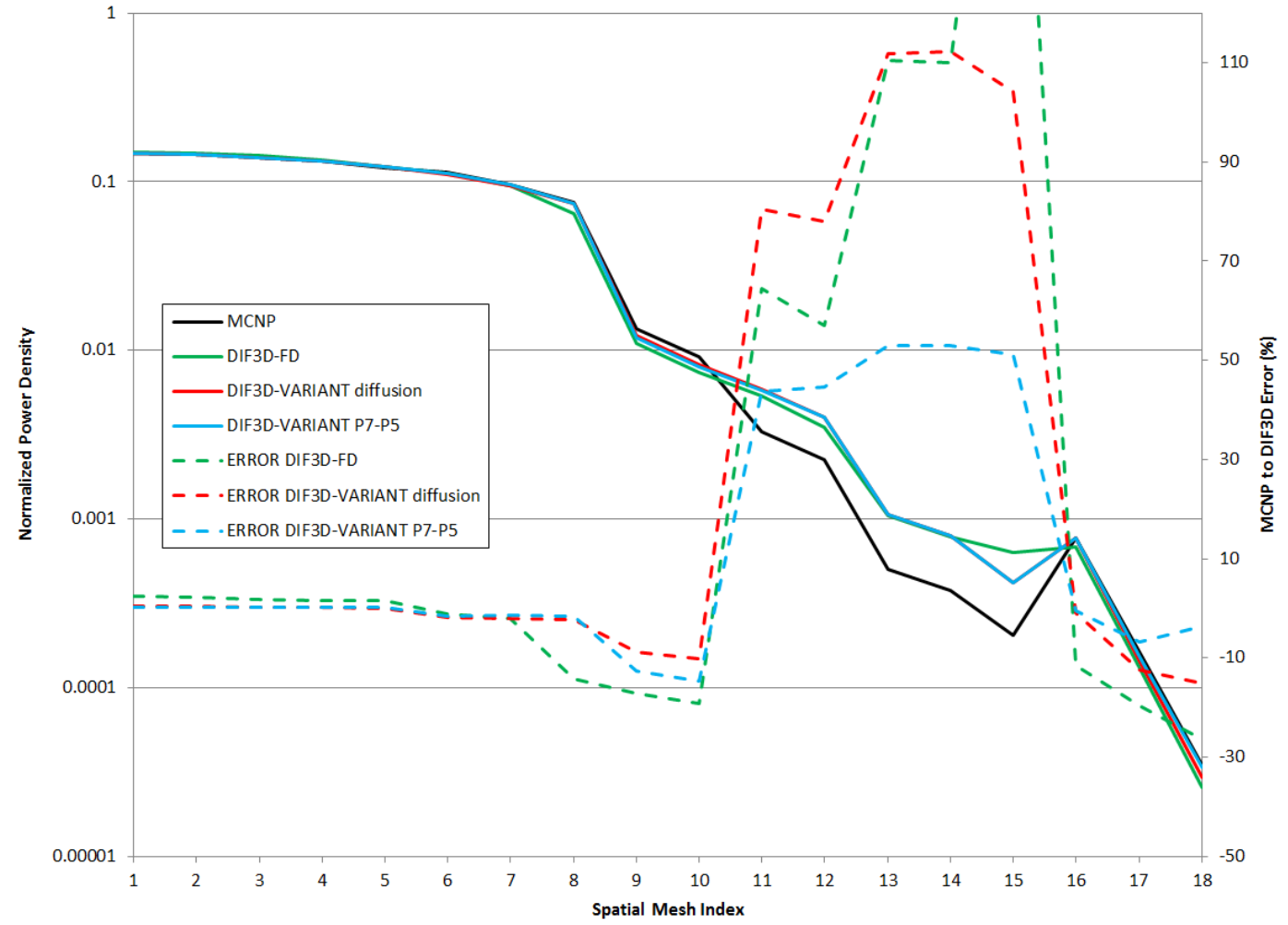

Figure 4.12. Neutron and Gamma Heating Results for Configuration 3. 
Overall, the outcome of configuration 3 indicates quite a different story from that seen in the previous two configurations. Because the control rod, blanket, reflector, and shield compositions are identical between all three configurations, the previous bias that was seen in configurations 1 and 2 are hard to assert as a fundamental problem with the B-10, B-11, and C-12 gamma data. Instead, we must inspect the neutron and gamma flux solutions to understand what is causing the differences. To begin, Figure 4.13 shows the normalized gamma spectrum differences in the core center of configurations 2 and 3. As can be seen, there is a considerable difference in magnitude of gamma production in energies around 1 $\mathrm{MeV}$ (groups 11 to 13 ) which is directly attributable to the change in isotopes. We can look at the spatial distribution of the gamma sources in these groups which is provided in Figure 4.14. It is important to note that for each energy range, the color scale for both configurations 2 and 3 problems is identical such that the normalization differences seen in Figure 4.13 can be seen.

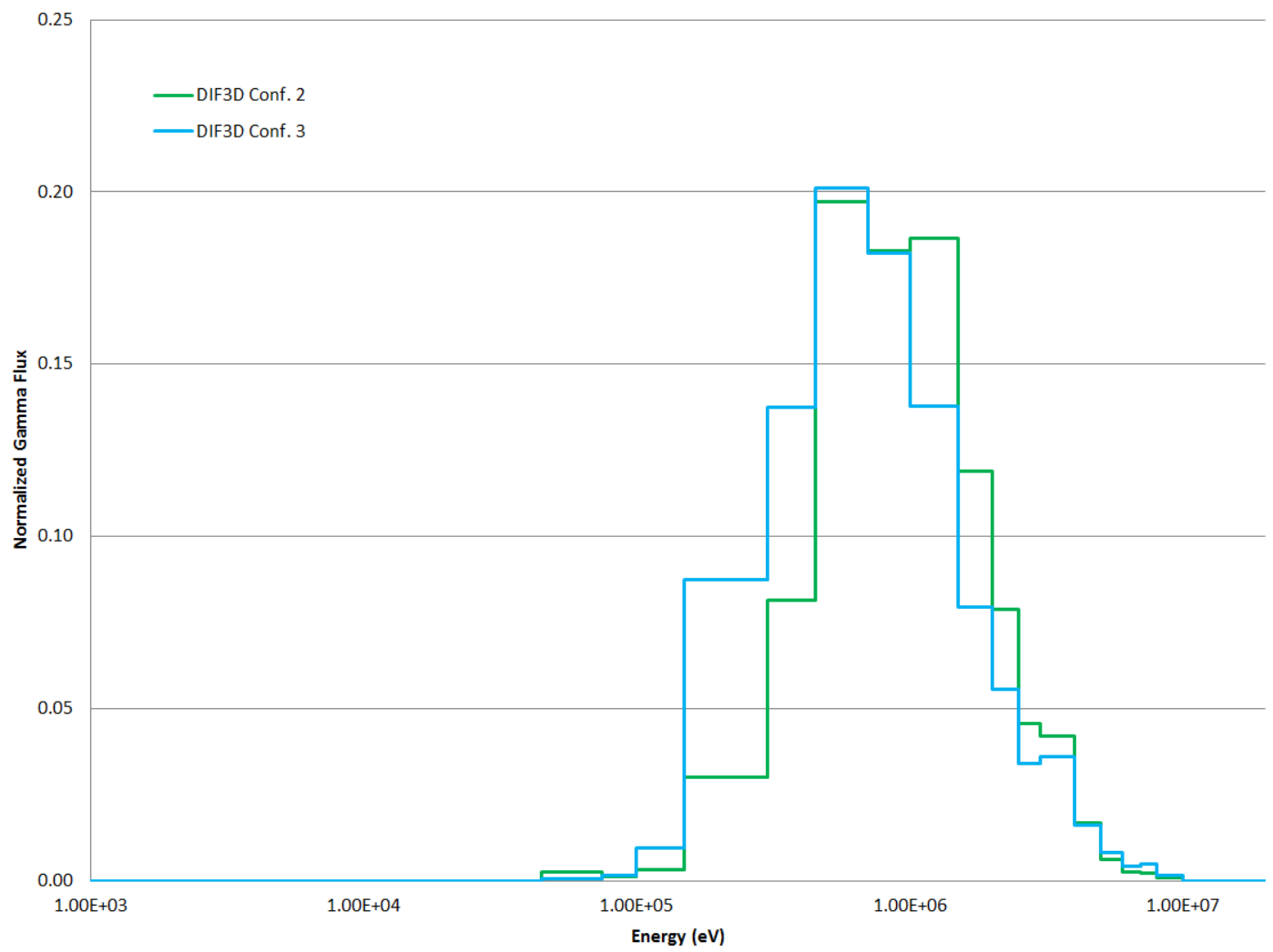

Figure 4.13. DIF3D Core Center Gamma Flux Spectrum in Configurations 2 and 3.

In each of the three pictures of Figure 4.14, one can see that the primary reason for the core center gamma production differences is the different enrichments. However, given that this is the peak of the gamma production in both systems, it should be apparent that the distribution changes will impact the gamma heating results in all of the control rod, blanket, reflector, and shielding regions at the selected tally positions. This is why the trend in the results changes between configurations 1 and 3. In effect, the first two problems are so similar that the errors appearing in similar regions seemed likely to be a systematic error. But when we alter the gamma emission spectrum and power distribution, we actually expose the true 
source of errors which is the inadequate energy resolution of the gamma data (and potentially some amount of systematic error in the cross section data processing).

Overall, we should redo all of these calculations using a finer energy structure in both neutrons and gammas to assess the impact upon the power prediction. While it is unlikely that the errors between MCNP and DIF3D will go to zero, the general trend should indicate that the error is reducing. At present we cannot refine the energy structure in $\mathrm{MC}^{2}-3$ and this manuscript will be updated as additional detail and data are obtained.

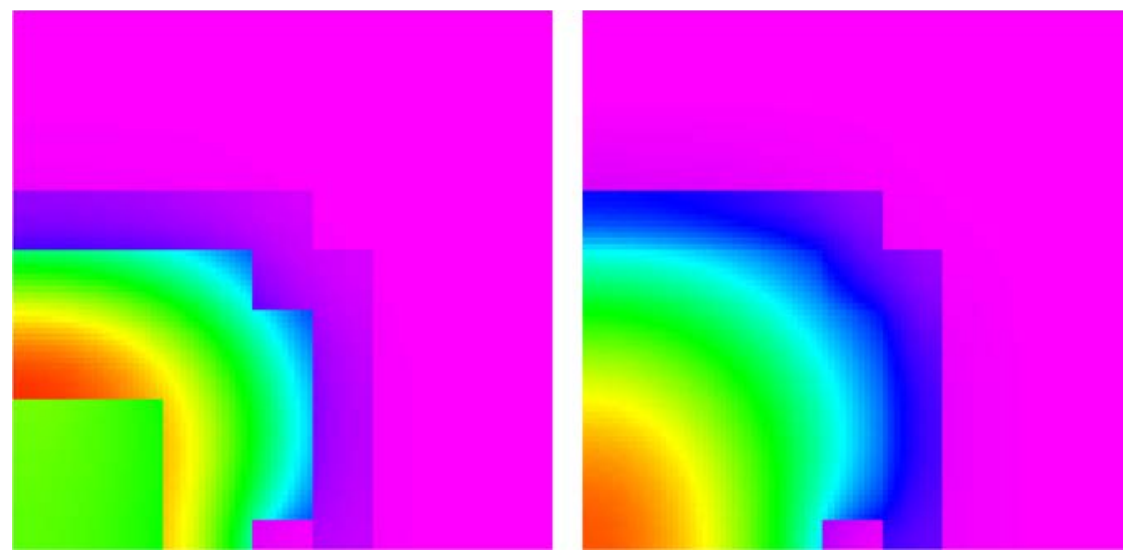

Group 11: $1 \mathrm{MeV}-1.5 \mathrm{MeV}$
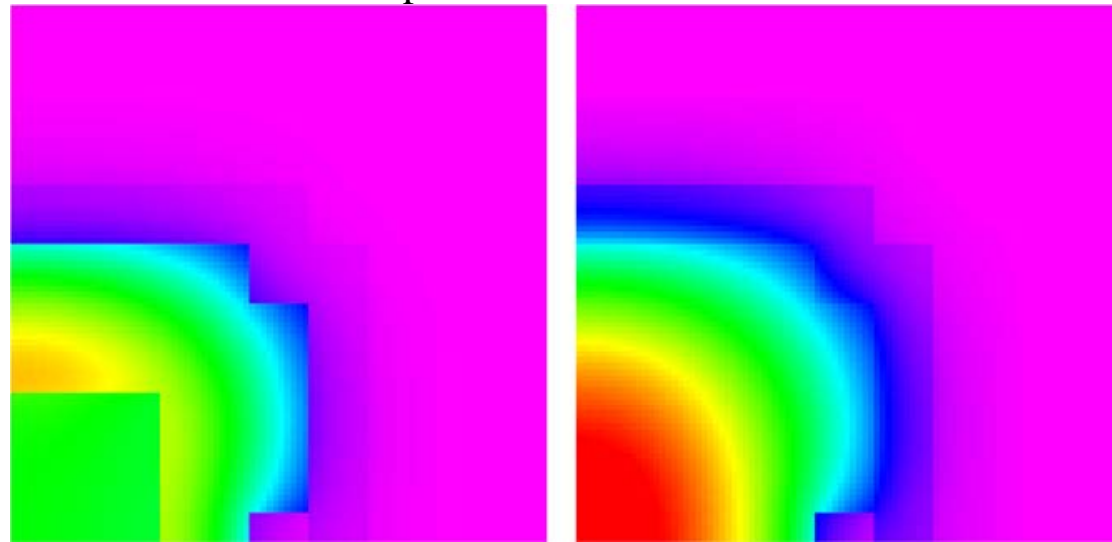

Group 12: $700 \mathrm{keV}-1 \mathrm{MeV}$
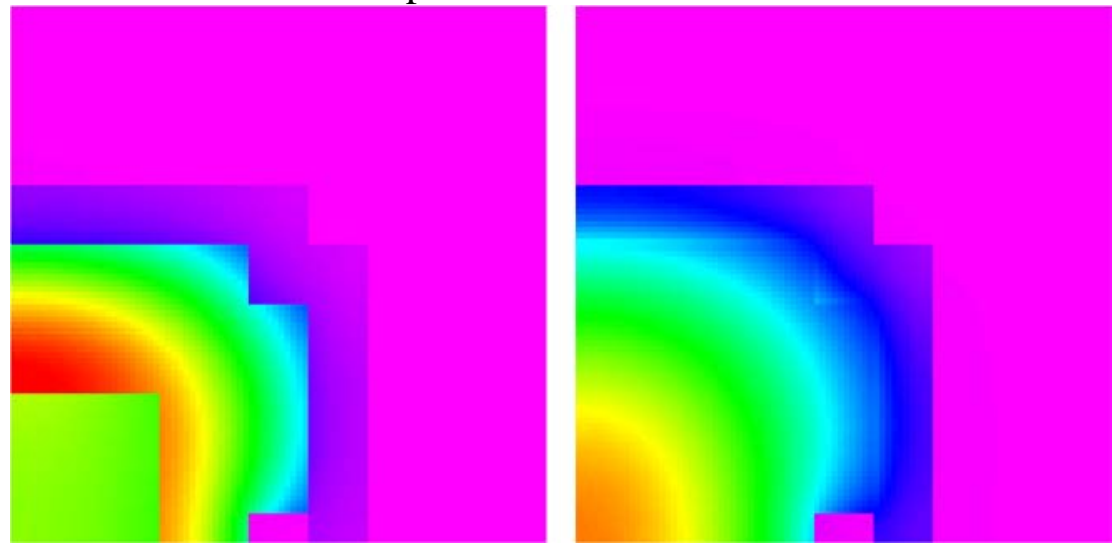

Group 13: $450 \mathrm{keV}$ - $700 \mathrm{keV}$

Figure 4.14. Gamma Sources for Configurations 2 and 3 Plotted on Identical Scales. 


\subsection{EBR-II Verification Problems}

While a ZPR or ZPPR problem is preferred for validation of GAMSOR, we did not have time to complete a detailed validation problem as part of this work and instead reiterate past verification work done using GAMSOR on Experimental Breeder Reactor II (EBR-II). The GAMSOR code was originally developed to assist in the analysis of EBR-II [10] and was part of the overall EBR-II program in DOE. The primary need for GAMSOR is to properly predict the distribution of power generation in a fast reactor system and some of the later EBR-II designs which removed the blankets required more attention be paid to the gamma heating of non-fuel assemblies.

The following work is taken directly from references [18] and [19] is the finished work of a Coordinated Research Project (CRP) on EBR-II Shutdown Heat Removal Test (SHRT) established by the International Atomic Energy Agency (IAEA). The primary focus of both documents is the demonstration of $\mathrm{MC}^{2}-3$ and DIF3D analysis via GAMSOR and comparisons against MCNP solutions (MCNP 6.1 was used with ENDF/B-VII.0 cross section data). Figure 4.15 shows the core configuration of the EBR-II where it is important to note that the blankets were placed outside of the reflectors. Since some of the EBR-II subassemblies were loaded with non-fissile pins, the gamma heat generation becomes the major contributor to the thermal power in those assemblies making this a good test of GAMSOR interior to a real reactor problem. The remaining details on geometry can be found elsewhere [20-21].

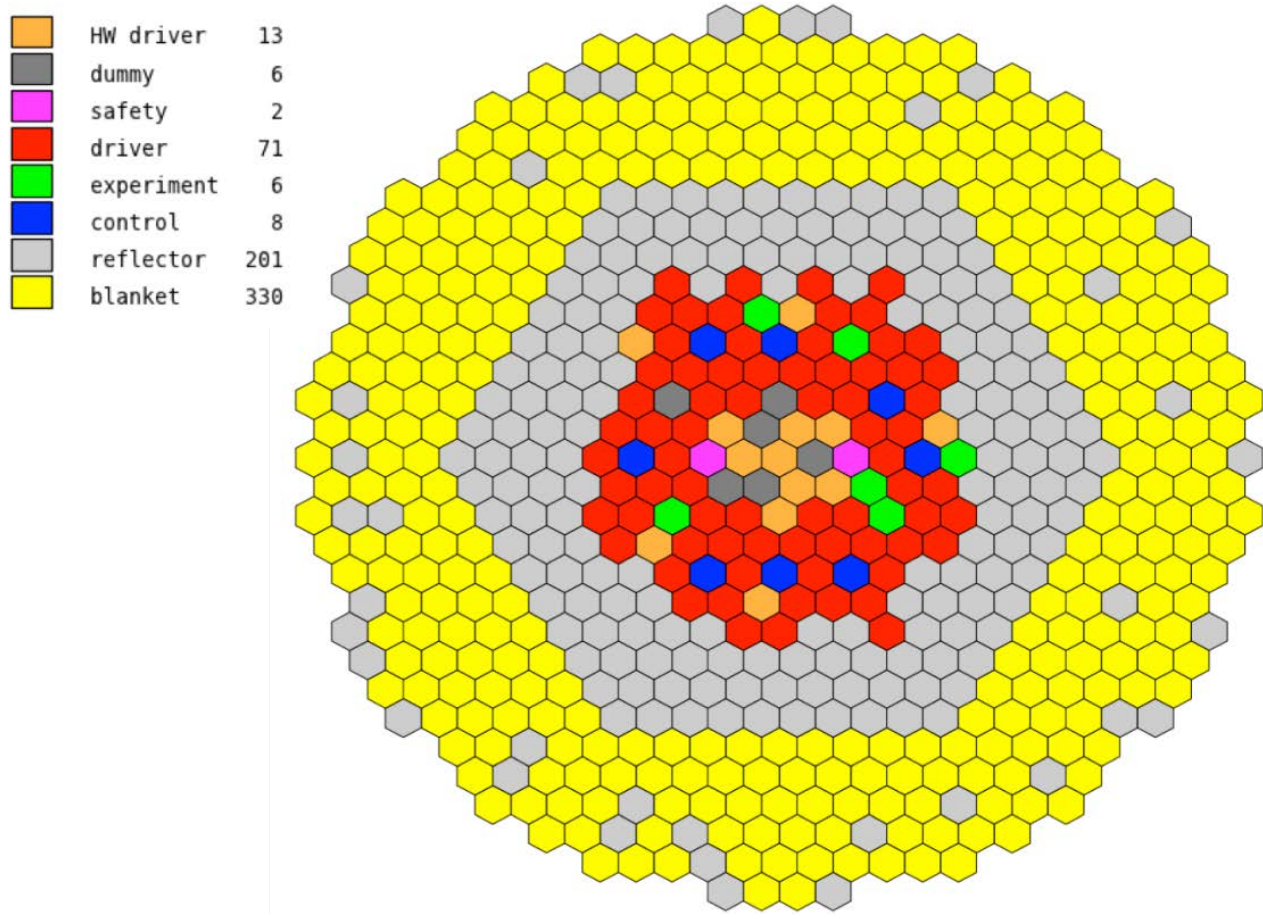

Figure 4.15. EBR-II Core Loading Pattern for Run 138B.

The TWODANT option of generating region wise cross sections was used with $\mathrm{MC}^{2}-3$ where an equivalent R-Z model was constructed of this EBR-II geometry. Both critical and rodded configurations of this loading were performed. Table 4.9 shows the keff results for the 
EBR-II problem where the same ENDF/B-VII.0 library was used in DIF3D and MCNP. A 33 group neutron structure and 21 group neutron structure were chosen for this analysis work. Both DIF3D and MCNP utilize homogenized assembly geometry to guarantee consistency in the results.

Table 4.10. EBR-II Run 138B keff Results.

\begin{tabular}{|c|c|c|}
\hline \multirow{2}{*}{ Code } & \multicolumn{2}{|c|}{$\mathrm{k}_{\text {eff }}$} \\
\cline { 2 - 3 } & Critical & Fully Inserted \\
\hline DIF3D-VARIANT diffusion 33 group & 0.96139 & 0.93041 \\
\hline DIF3D-VARIANT P3-P3 33 group & 0.98759 & 0.95537 \\
\hline MCNP & $0.99367 \pm 0.00002$ & $0.96188 \pm 0.00002$ \\
\hline
\end{tabular}

As can be seen, there is a considerable difference between the three results in Table 4.10 which was not the case in the previous verification problems. Diffusion theory is considerably off from MCNP, over $3000 \mathrm{pcm}$, which is attributable to the removal of blankets that introduces considerable leakage into the system. As a consequence, the transport option in DIF3D-VARIANT is required and the diffusion results are ignored. In general the $\sim 600$ pcm error between the DIF3D transport solution and MCNP will not lead to substantial errors in flux or power details noting that, based upon the previous 2D verification problems, a less than 200 pcm error is likely achievable given additional refinements in the space-angleenergy approximations in the deterministic model.

The difference between assembly power calculated by DIF3D and MCNP is presented in Figure 4.16 for the assemblies in the inner 7 rings of the core excluding the reflectors. The results from MCNP were normalized by the total power of the first 7 rings (excluding the reflectors) calculated by DIF3D. The maximum statistical uncertainty of the assembly power reported by MCNP is $0.34 \%$ which is sufficiently small to be considered accurate.

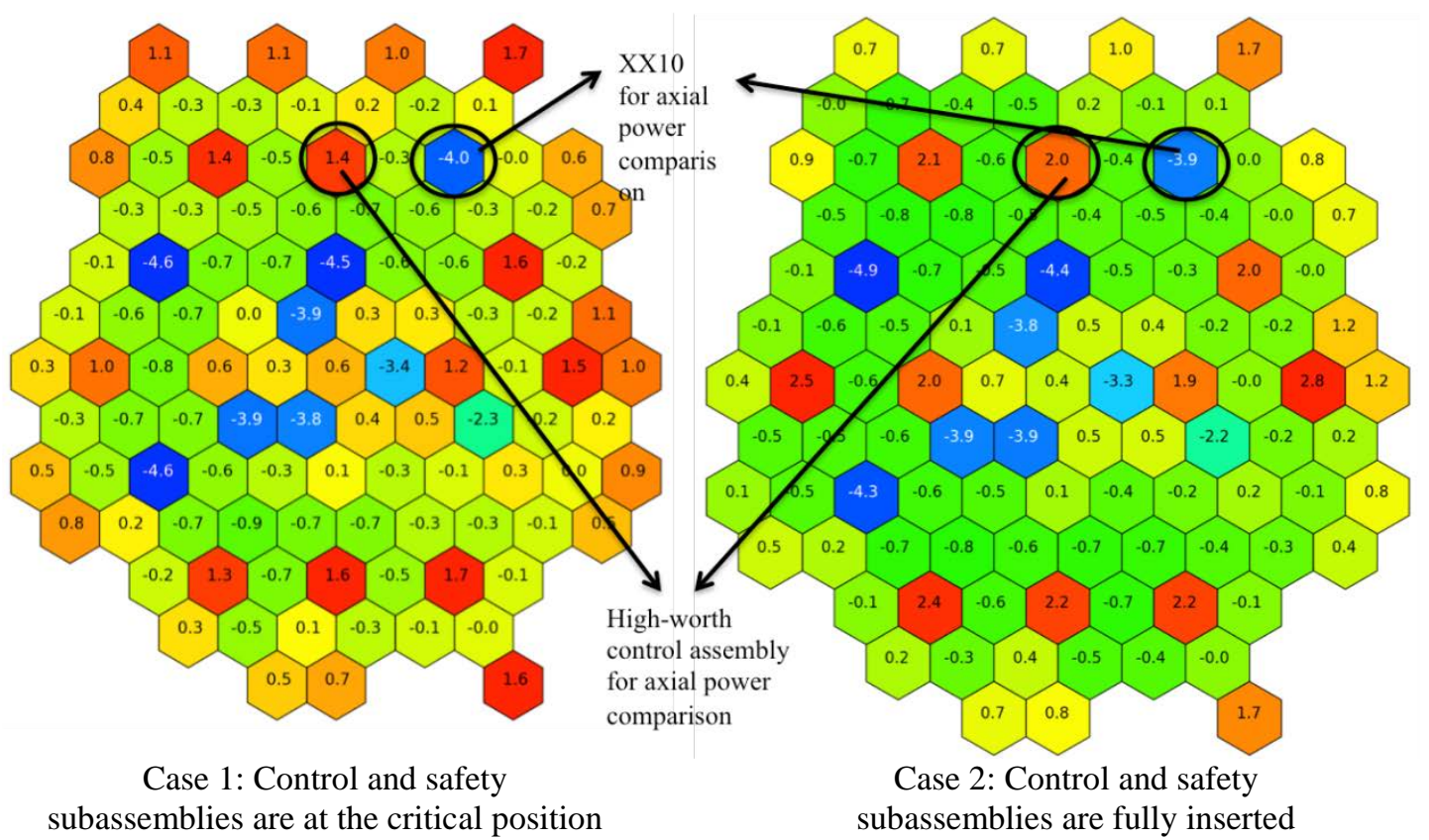

Figure 4.16. Power Difference (\%) for Inner-Core Assemblies between DIF3D and MCNP. 
As can be seen in Figure 4.16, the difference between the DIF3D and MCNP calculated powers in the dummy assemblies XY16, X320C, and XX10 range from $-2.2 \%$ to $-4.9 \%$. In this case, DIF3D tends to underestimate the power generated in the structural assemblies; however, the magnitude of underestimation becomes much smaller compared to the DIF3D calculation without using GAMSOR (i.e. ignoring gamma transport).

The XX10 assembly and a high-worth control assembly are highlighted in Figure 4.16 as they were chosen for an additional axial power distribution comparison between DIF3D and MCNP. The power density in each axial region of XX10 and the high-worth control assembly are summarized in Table 4.11 and Table 4.12, respectively. Starting with Table 4.11, one can see that the power density is computed for the core level structural component and the upper and lower grid and reflector regions. In this assembly, there is no fission generation and thus the power is low relative to a powered assembly (see Table 4.12). The MCNP and DIF3D solutions are very comparable with a maximum 7\% error in the power prediction which is very good considering the rather low power levels in some of the regions. Without using GAMSOR, the power level in these regions is an order of magnitude less leading to errors of nearly $100 \%$.

Looking at Table 4.12, the core level power is considerably higher than that seen in the non-fuel assembly of Table 4.11. The power density prediction in the core region is very similar between MCNP and DIF3D, which is consistent with that observed with the simple 2D benchmark problems of the previous section. Below the core we see a considerable increase in the error, but this tends to correlate well with lower power levels which are susceptible to the normalization process and the MCNP statistical error on the larger powers. One particularly interesting aspect to note is the power density prediction in the fully inserted safety subassembly where the core region is still producing a significant amount of power as is the poison region.

Table 4.11. Axial Power Density $\left(\mathrm{W} / \mathrm{cm}^{3}\right)$ in the XX10 Assembly

\begin{tabular}{|l|r|r|r|r|}
\hline \multicolumn{1}{|c|}{ Region } & DIF3D & MCNP6 & $\begin{array}{c}\text { MCNP6 } \\
\text { Uncertainty }\end{array}$ & Difference \\
\hline Control and safety subassembly at critical position & \multicolumn{2}{c|}{} \\
\hline Lower Reflector & 1.52 & 1.59 & $0.59 \%$ & $-4.09 \%$ \\
\hline Lower Grid & 2.19 & 2.23 & $0.65 \%$ & $-1.68 \%$ \\
\hline XX10 & 6.71 & 7.11 & $0.21 \%$ & $-5.69 \%$ \\
\hline Upper Grid & 0.38 & 0.39 & $1.63 \%$ & $-3.25 \%$ \\
\hline Upper Reflector & 0.50 & 0.53 & $1.43 \%$ & $-7.15 \%$ \\
\hline Control and safety subassembly are fully inserted & \multicolumn{3}{|c|}{} \\
\hline Lower Reflector & 1.58 & 1.67 & $0.59 \%$ & $-5.32 \%$ \\
\hline Lower Grid & 2.20 & 2.26 & $0.65 \%$ & $-2.60 \%$ \\
\hline XX10 & 7.05 & 7.32 & $0.21 \%$ & $-3.69 \%$ \\
\hline Upper Grid & 0.43 & 0.45 & $1.63 \%$ & $-5.09 \%$ \\
\hline Upper Reflector & 0.62 & 0.64 & $1.43 \%$ & $-2.98 \%$ \\
\hline
\end{tabular}


Table 4.12. Axial Power Density $\left(\mathrm{W} / \mathrm{cm}^{3}\right)$ in the High-Worth Control Assembly

\begin{tabular}{|l|r|r|r|r|}
\hline \multicolumn{1}{|c|}{ Region } & \multicolumn{1}{c|}{ DIF3D } & \multicolumn{1}{c|}{ MCNP6 } & $\begin{array}{c}\text { MCNP6 } \\
\text { Uncertainty }\end{array}$ & Difference \\
\hline Control and safety subassembly at critical position \\
\hline Lower Reflector & 0.77 & 0.83 & $1.19 \%$ & $-6.61 \%$ \\
\hline Lower Grid & 0.78 & 0.77 & $1.23 \%$ & $0.37 \%$ \\
\hline Fuel & 349.97 & 344.65 & $0.14 \%$ & $1.54 \%$ \\
\hline Gas Plenum & 5.66 & 6.00 & $0.29 \%$ & $-5.66 \%$ \\
\hline Poison & 7.43 & 7.66 & $0.21 \%$ & $-2.99 \%$ \\
\hline Upper Reflector & 0.33 & 0.37 & $2.06 \%$ & $-10.24 \%$ \\
\hline Control and safety subassembly fully inserted \\
\hline Lower Reflector & 0.35 & 0.36 & $1.19 \%$ & $-3.88 \%$ \\
\hline Lower Grid & 0.28 & 0.29 & $1.23 \%$ & $-1.82 \%$ \\
\hline Fuel & 232.68 & 227.18 & $0.14 \%$ & $2.42 \%$ \\
\hline Gas Plenum & 4.66 & 4.90 & $0.29 \%$ & $-4.90 \%$ \\
\hline Poison & 14.20 & 14.57 & $0.21 \%$ & $-2.58 \%$ \\
\hline Upper Reflector & 0.88 & 0.95 & $2.06 \%$ & $-7.96 \%$ \\
\hline
\end{tabular}

Except for the keff comparison, the preceding EBR-II results are consistent with the simple 2D problem results presented in the previous section. In this case we present a code to code comparison for a real operating state of a real reactor and still demonstrate that GAMSOR with $\mathrm{MC}^{2}-3$ produces results that are comparable to MCNP. 


\section{Gamma Library Generation with NJOY}

The NJOY code [22] is used to generate the prompt gamma production matrices and the gamma interaction cross sections as well as the neutron and gamma heating factors at the infinite dilute condition and $300 \mathrm{~K}$. The prompt gamma production matrices are generated using the RECONR, BROADR, HEATR and GROUPR modules of NJOY and the gamma interaction cross sections using the RECONR and GAMINR modules of NJOY and the 1/E weighting spectrum. The GAMINR module of NJOY provides cross sections for four different reactions from the ENDF photo-atomic sub-library. The resulting NJOY output contains gamma interaction cross section vectors (coherent, incoherent, pair production, and photoelectric cross sections, and gamma KERMA factors), neutron-induced photon production matrices, neutron and gamma heating and damage factors as well as neutron cross sections of an isotope. Figure 5.1 shows an overview of the gamma library generation using NJOY.

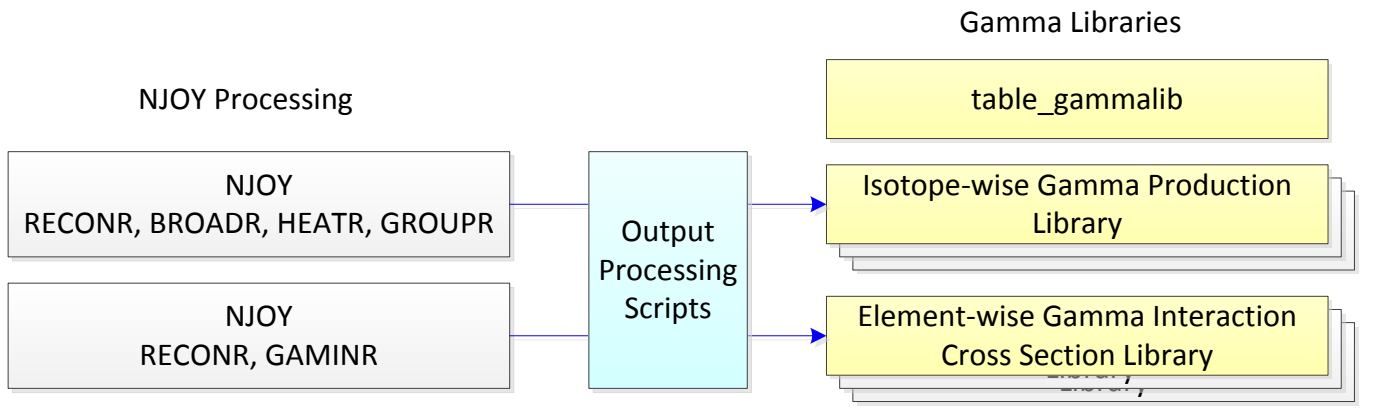

Figure 5.1. Generation of Gamma Libraries Using NJOY

While the production matrices for non-elastic neutron scattering reactions are directly used, those for capture and fission reactions are converted into the yield matrices, as shown below, in order to take into account the self-shielding effect on gamma production.

$Y_{x}^{i}(g \rightarrow G G)=\sigma_{x}^{i}(g \rightarrow G G) / \sigma_{x g}^{i}$

where $\sigma_{x g}^{i}$ is the infinite dilute cross section of reaction $x$ (fission or capture) of isotope $i$ for neutron ultrafine group $g$, and $\sigma_{x}^{i}(g \rightarrow G G)$ is the production cross section matrix reaction $x$ of isotope $i$ from neutron ultrafine group $g$ to gamma broad group $G G$.

It should be noted that the KERMA factors of neutron and gamma are included in the gamma production library (generated from HEATR) and the gamma interaction cross section library (generated from GAMINR), respectively. The self-shielding effect on the KERMA factors of neutron reactions needs to be accounted for in the same manner as the gamma production matrices. However, this is not considered in the current version. The delayed gamma production and damage factor are not included at the moment, either. The selfshielding effect on the KERMA factors of neutron reactions, delayed gamma production, and damage factor will be included in the new version of gamma libraries, which will be based on the Cross Section Evaluation Working Group (CSEWG) 94-group gamma groups [22].

The gamma libraries of MC2-3 were generated based on the ultrafine neutron group $(2,082)$ and 21 gamma group structure. The 21-group structure, shown in 
Table 5.1, covers the energy range from $20 \mathrm{keV}$ to $20 \mathrm{MeV}$, representing the smooth gamma interaction cross section energy intervals for the energy range of interest in the gamma heating calculation. The scattering of gamma ray is highly anisotropic as the mass of photon is negligible. Thus, the Legendre scattering matrices were generated up to $\mathrm{P}_{3}$ for the 21-group gamma interaction cross section library.

For the ENDF/B-VII.0 data [23], the gamma production matrices were generated for 393 isotopes and the gamma interaction cross sections were evaluated for 100 elements since gamma rays interact primarily with electrons. The scripts were developed to help generate NJOY inputs for each isotope (HEATR) or element (GAMINR) and to process NJOY outputs to produce the gamma production and interaction cross section library files for individual isotopes in the ASCII format. The file structures of the isotopic gamma production and element-wise interaction cross section libraries are shown in 
Table 5.2 and Table 5.3, respectively. The file names for isotopes are specified in the separate index file named table_gammalib in the format shown in

Table 5.4.

Table 5.1. Upper Energy Boundaries (eV) of the 21-group Structure of Gamma

\begin{tabular}{|c|c|c|c|}
\hline Group & Energy & Group & Energy \\
\hline 1 & $2.0000 \mathrm{E}+07$ & 11 & $1.5000 \mathrm{E}+06$ \\
2 & $1.0000 \mathrm{E}+07$ & 12 & $1.0000 \mathrm{E}+06$ \\
3 & $8.0000 \mathrm{E}+06$ & 13 & $7.0000 \mathrm{E}+05$ \\
4 & $7.0000 \mathrm{E}+06$ & 14 & $4.5000 \mathrm{E}+05$ \\
5 & $6.0000 \mathrm{E}+06$ & 15 & $3.0000 \mathrm{E}+05$ \\
6 & $5.0000 \mathrm{E}+06$ & 16 & $1.5000 \mathrm{E}+05$ \\
7 & $4.0000 \mathrm{E}+06$ & 17 & $1.0000 \mathrm{E}+05$ \\
8 & $3.0000 \mathrm{E}+06$ & 18 & $7.5000 \mathrm{E}+04$ \\
9 & $2.5000 \mathrm{E}+06$ & 19 & $4.5000 \mathrm{E}+04$ \\
10 & $2.0000 \mathrm{E}+06$ & 20 & $3.0000 \mathrm{E}+04$ \\
& & 21 & $2.0000 \mathrm{E}+04$ \\
\hline
\end{tabular}


Table 5.2. Structure of the Prompt Gamma Production Library of Each Isotope

\begin{tabular}{|l|l|}
\hline \multicolumn{1}{|c|}{ Row } & \multicolumn{1}{c|}{ Content } \\
\hline 1 & $\begin{array}{l}\text { Number of neutron groups (NG), number of gamma groups (GG) } \\
\text { (Neutron group structure) } \\
\text { Group, (info), upper energy boundaries (eV) of neutron energy groups }\end{array}$ \\
\hline+ NG & $\begin{array}{l}\text { Gamma group structure) } \\
\text { Group, (info), upper energy boundaries (eV) of gamma energy groups }\end{array}$ \\
\hline+1 & $\begin{array}{l}\text { Number of absorption (nabs), fission (nfis), non-elastic scattering (nsct) } \\
\text { contribution data }\end{array}$ \\
\hline $\begin{array}{l}\text { If nabs }>0, \\
+ \text { nabs }\end{array}$ & $\begin{array}{l}\text { (Absorption contributions from neutron NG to gamma GG) } \\
\text { Neutron group, initial gamma group (igb), ending gamma group (ige), (info), } \\
\text { multiplier, absorption (ig = igb, ige) }\end{array}$ \\
\hline $\begin{array}{l}\text { If nfis }>0, \\
+ \text { nfis }\end{array}$ & $\begin{array}{l}\text { (Fission contributions from neutron NG to gamma GG) } \\
\text { Neutron group, first gamma group (igb), last gamma group (ige), (info), } \\
\text { multiplier, fission (from igb to ige) }\end{array}$ \\
\hline $\begin{array}{l}\text { If nsct }>0, \\
+ \text { nsct }\end{array}$ & $\begin{array}{l}\text { (Non-elastic contributions from neutron NG to gamma GG) } \\
\text { Neutron group, first gamma group (igb), last gamma group (ige), (info), } \\
\text { multiplier, scattering (ig = igb, ige) }\end{array}$ \\
\hline+ NG & $\begin{array}{l}\text { (KERMA factor) } \\
\text { Neutron group, (info), KERMA factor (eV-barns) }\end{array}$ \\
\hline
\end{tabular}

Table 5.3. Structure of the Gamma Interaction Cross Section Library of Each Isotope

\begin{tabular}{|l|l|}
\hline \multicolumn{1}{|c|}{ Row } & \multicolumn{1}{c|}{ Content } \\
\hline 1 & $\begin{array}{l}\text { Number of gamma groups (GG), scattering order (norder), scattering matrix option } \\
\text { Option = 1: total scattering matrix only } \\
\text { Option = 2: incoherent + coherent matrix and separate pair production matrix } \\
\text { Option = 3: all separate incoherent, coherent, and pair production matrices }\end{array}$ \\
\hline+ GG & $\begin{array}{l}\text { (Principal gamma cross sections) } \\
\text { Gamma group, energy (eV), total cross section, (info), total scattering cross section, } \\
\text { coherent scattering cross section, incoherent scattering cross section, pair-production } \\
\text { cross section, absorption cross section, heating (eV-barns) }\end{array}$ \\
\hline+ norder & $\begin{array}{l}\text { (Number of lines = norder } \times \text { GG) } \\
\text { First gamma group (igb), last gamma group (ige), total scattering cross section, } \\
\text { scattering matrix from igb to ige }\end{array}$ \\
\hline
\end{tabular}

Table 5.4. Structure of the Index File table_gammalib

\begin{tabular}{|l|l|}
\hline \multicolumn{1}{|c|}{ Row } & \multicolumn{1}{c|}{ Content } \\
\hline 1 & Number of total lines (L) \\
\hline$+\mathrm{L}$ & $\begin{array}{l}\text { Sequential number, isotope ID, gamma production library file name, gamma } \\
\text { interaction cross section library file name }\end{array}$ \\
\hline
\end{tabular}




\section{Conclusions}

The GAMSOR code was built to support the EBR-II analysis and operations work in the mid 1980s. The intention was to properly account for the gamma heating in an operating reactor core on core internals. In conventional reactor physics methods, the gamma heating component is ignored such that the gamma absorption is forced to occur at the emission site. For experimental reactor systems like EBR-II and FFTF, the placement of structural pins and assemblies internal to the core leads to problems with power heating predictions because there is no fission power source internal to the assembly to dictate a spatial distribution. Thus, a gamma heating calculation is needed where gamma production from fission is required to define the correct source. To fix this oversight, the GAMSOR code was created.

As shown in this manuscript, to execute GAMSOR, one must execute three separate DIF3D calculations which are called the GAMSOR sequence. The first DIF3D calculation is a conventional neutron diffusion (or transport) calculation with a modified version of the DIF3D executable. In this case one obtains the neutron flux as if all of the gamma emission was immediately absorbed at the emission point. The purpose of the modified DIF3D executable, called dif3d_gamsor.x, is to take the neutron flux and compute the spatial distribution of the gamma source.

As discussed, the gamma flux in a conventional fission reactor system does not perturb the neutron flux and thus the gamma flux calculation can be cast as a fixed source problem given a solution to the steady state neutron problem. The second DIF3D calculation in the GAMSOR sequence is done to obtain the gamma flux distribution. It requires substantial modifications to the A.DIF3D input section to guarantee convergence as DIF3D was never setup to be a proper fixed source solver.

After the first two steps in the GAMSOR sequence is done, one has both the neutron and gamma flux solution for a given problem. The third step in the GAMSOR sequence is to use the SUMMARY module of DIF3D to compute the neutron and gamma heating distributions which are stored in the binary files NPDINT and GPDINT. The combined result is the actual power distribution which is stored in PWDINT. It is important to note that the power level rarely matches the desired power setting chosen in the original neutronics problem as the gamma flux calculation generally leads to a substantial amount of leakage from the modeled domain. In this case, the user must rerun the calculation or renormalize the fluxes to obtain the desired power rating for the modeled domain.

In this manuscript, we demonstrated the GAMSOR code on an analytical benchmark, a series of simple 2D test problems, and an EBR-II configuration test problem. Additional validation is possible using ZPPR TLD measurements but that work is deferred as part of later work. The 2D test problems and EBR-II problems all rely upon MCNP to obtain a reference solution. The 2D comparisons demonstrated very good results for the eigenvalue solution and neutron flux solutions, but mixed results for the power heating and gamma flux results. An inspection of the results indicated that the gamma flux energy discretization is insufficient near the $1 \mathrm{MeV}$ peak emission point. It is important to note that it is not clear at this time whether refinements in energy will guarantee improvements in the comparisons between MCNP and DIF3D for the gamma flux and power heating results. The EBR-II calculations 
with DIF3D and MCNP are the next logical step past the simple 2D tests. In this case, we know the problem being studied is a real reactor configuration that was operated under the EBR-II work. Unlike the 2D cases, the eigenvalue results were considerably off, $>2000 \mathrm{pcm}$ while the transport calculations were $\sim 600 \mathrm{pcm}$ off. Although these are substantial errors, the core specific power distribution (i.e. ignoring blankets, reflectors, and shielding) shows that DIF3D produces power heating results very consistent with those of MCNP. Much like the 2D results, one must apply higher order space-angle-energy refinements to improve the accuracy with respect to MCNP.

This manuscript covers the details of how GAMSOR works and how it should be used. It is important for users to follow the guidance on using DIF3D to solve fixed source problems. There now exist sufficient test problems in GAMSOR to demonstrate good codeto-code comparisons with MCNP although additional work is required to reduce the errors further as necessary. While validation is important, it will have to be included later as available to demonstrate that the underlying methodology yields results consistent with measurements taken in reactor systems. User supports and questions on GAMSOR can be requested via nera-software@anl.gov. 


\section{Appendix A. Quick Reference GAMSOR Guidance}

When using the GAMSOR code, the user should follow the procedure outlined by Figure 3.4. The various guidance and warnings mentioned throughout this manuscript are summarized in Table 0.1

Table 0.1. GAMSOR Usage Quick Reference

\begin{tabular}{|c|c|}
\hline Key Data from Figure 3.4 & Description \\
\hline PMATRX & $\begin{array}{l}\text { - Contains the gamma emission rates from neutron } \\
\text { interactions for each isotope. } \\
\text { - This data has been observed to be non-physical for some } \\
\text { actinides in that higher energy gamma rays have higher } \\
\text { interaction probability than lower energy gamma rays. } \\
\text { - The production matrices will not sum to the total merged } \\
\text { interaction probability as the equivalent v value for gamma } \\
\text { emission during fission is embedded. }\end{array}$ \\
\hline $\begin{array}{l}\text { dif3d_gamsor.x } \\
\text { (neutron flux) }\end{array}$ & $\begin{array}{l}\text { - This modified version of dif3d.x will use the PMATRX } \\
\text { data to construct the fixed source (FIXSRC or VARSRC) } \\
\text { that is needed for the gamma flux calculation. } \\
\text { - The standard DIF3D output edits from this step will be } \\
\text { identical to those generated by dif3d.x } \\
\text { - The GAMSOR “gamma power" edits reported by this } \\
\text { executable are based upon erroneous energy deposition } \\
\text { predictions and should be ignored. }\end{array}$ \\
\hline $\begin{array}{l}\text { GAMISO or Gamma } \\
\text { ISOTXS }\end{array}$ & $\begin{array}{l}\text { - Contains the microscopic gamma interaction cross sections } \\
\text { for each isotope } \\
\text { Because ISOTXS does not have storage positions for } \\
\text { photon interactions, the capture storage position is used for } \\
\text { all photon cross section data. In recent versions of } \mathrm{MC}^{2}-3 \text {, } \\
\text { the pair production term was separated and stored into the } \\
\text { (n,2n) storage position to prevent negative capture cross } \\
\text { sections from appearing }\end{array}$ \\
\hline dif3d.x (gamma flux) & $\begin{array}{l}\text { - The iterative flux error in DIF3D-FD (default 0.04) should } \\
\text { be set to } 10^{-7} \text {. } \\
\text { - The radial and axial inner iteration limits on DIF3D-Nodal } \\
\text { and DIF3D-VARIANT should be set to a minimum of } 10 \\
\text { each. } \\
\text { - The ANIP3 input geometry from step } 1 \text { should be repeated } \\
\text { in this step in case the isotope ordering in GAMISO } \\
\text { (gamma) versus ISOTXS (neutron) is not consistent. } \\
\text { - The use of FIXSRC for DIF3D-Nodal or DIF3D- } \\
\text { VARIANT constitutes a coarsening of the gamma source } \\
\text { and thus an additional level of approximation. VARSRC is } \\
\text { provided for DIF3D-VARIANT as a viable solution. } \\
\text { There are no error checks on the flux and this step should }\end{array}$ \\
\hline
\end{tabular}




\begin{tabular}{|l|l|}
\hline & $\begin{array}{l}\text { be repeated for any given problem size to ensure the flux is } \\
\text { converged sufficiently when not using DIF3D-FD. } \\
\text { - }\end{array}$ \\
\hline dif3d.x (summary) & ill regular DIF3D edits except for flux edits should be \\
& ignored. All flux edits refer to the gamma flux values. \\
\hline & The power level is not normalized to the user requested \\
input in A.DIF3D, but typically less due to the leakage of \\
gammas from the modeled domain. \\
- $\begin{array}{l}\text { The standard DIF3D edits will be repeats of the outputs } \\
\text { from dif3d_gamsor.x or dif3d.x. They will not consider } \\
\text { any aspect of the gamma flux solution or power }\end{array}$ \\
redistribution associated with it. Part of the SUMMAR \\
edits do include the gamma heating correctly. \\
The important output of GAMSOR are the files PWDINT, \\
NPDINT, and GPDINT which give the mesh wise power \\
distribution information for total, neutron, and gamma \\
respectively.
\end{tabular}




\section{References}

1. K. L. Derstine, "DIF3D: A Code to Solve One-, Two-, and Three-Dimensional FiniteDifference Diffusion Theory Problems,” ANL-82-64, Argonne National Laboratory (1984).

2. G. Palmiotti, E. E. Lewis, and C. B. Carrico, "VARIANT: VARIational Anisotropic Nodal Transport for Multidimensional Cartesian and Hexagonal Geometry Calculations," ANL-95/40, Argonne National Laboratory (1995).

3. I. Dilber and E. E. Lewis, "Variational Nodal Methods for Neutron Transport," Nucl. Sci. Eng., 91, 132 (1985).

4. C. B. Carrico, E. E. Lewis, and G. Palmiotti, "Three-Dimensional Variational Nodal Transport Methods for Cartesian, Triangular, and Hexagonal Criticality Calculations,” Nucl. Sci. Eng., 111, 168 (1992).

5. M. A. Smith, N. Tsoulfanidis, E. E. Lewis, G. Palmiotti and T. A. Taiwo, "Higher Order Angular Capabilities of the VARIANT Code," Trans. Am. Nucl. Soc., 86, 321-322, (2002).

6. M. A. Smith, E. E. Lewis, E. R. Shemon, "DIF3D-VARIANT 11.0: A Decade of Updates,” ANL/NE-14/1, Argonne National Laboratory (2014).

7. E. E. Lewis and W. F. Miller Jr., "Computational Methods of Neutron Transport," New York: John Wiley \& Sons (1984).

8. C. H. Lee and W. S. Yang, "MC²-3: Multigroup Cross Section Generation Code for Fast Reactor Analysis,” ANL/NE-11/41 Rev. 1, Argonne National Laboratory, January (2012).

9. R. E. Macfarlane and A. C. Kahler, "Methods for Processing ENDF/B-VII with NJOY," Nuclear Data Sheet, 111, 2739 (2010).

10. M. A. Smith and R. N. Hill, Personal Communication (2014).

11. M. A. Smith and R. N. Hill, Personal Communication (2016).

12. L. C. Just, H. Henryson II, A. S. Kennedy, S. D. Sparck, B. J. Toppel and P. M. Walker, "The System Aspects and Interface Data Sets of the Argonne Reactor Computation (ARC) System," ANL-7711, Argonne National Laboratory (1971).

13. M. A. Smith, et al., "VARI3D \& PERSENT: Perturbation and Sensitivity Analysis," ANL/NE-13/8, Argonne National Laboratory (2013).

14. W. S. Yang and M. A. Smith, "RCT: REBUS Based Pin Power Reconstruction Using the DIF3D-Nodal and DIF3D-VARIANT Options,” ANL/NE-14/15, Argonne National Laboratory (2015).

15. M. A. Smith and R. N. Hill, Personal Communication (2016).

16. M. A. Smith and R. M. Lell, Personal Communication (2016).

17. R. M. Lell, J. A. Morman, R. W. Schaefer and R. D. McKnight, “ZPR-6 Assembly 7 High ${ }^{240} \mathrm{Pu}$ Core Experiments: A Fast Reactor Core With Mixed (Pu,U)-Oxide Fuel and a Central High ${ }^{240} \mathrm{Pu}$ Zone,” ZPR-LMFR-EXP-002, International Handbook of Evaluated Reactor Physics Benchmark Experiments, NEA/ NSC/DOC(2006)1, March (2009).

18. T. Fei, C. H. Lee, T. K. Kim, and T. Sofu, "Calculation of Photon Heat Generation for EBR-II Using DIF3D/MC²-3,” ANS Winter Meeting, Anaheim, California, November 913 (2014).

19. Personal communication with T. Fei, C. H. Lee, T. K. Kim, and M. A. Smith (2016).

20. T. Fei, A. Mohamed, T. K. Kim, and M. A. Smith Personal Communication January (2012). 
21. L. L. BRIGGS et al, "EBR-II Passive Safety Demonstration Tests Benchmark Analyses Phase 1,” Trans. Am. Nucl. Soc., 111, 1263 (2014).

22. R. E. MacFarlane and D. W. Muir, "NJOY99.0: Code System for Producing Pointwise and Multigroup Neutron and Photon Cross Sections from ENDF/B Data," PSR480/NJOY99.0, Oak Ridge National Laboratory (2000).

23. M. B. Chadwick et al., "ENDF/B-VII.0: Next Generation Evaluated Nuclear Data Library for Nuclear Science and Technology,” Science Direct, Nuclear Data Sheets 107, 29313060 (2006).

24. Los Alamos Scientific Laboratory Group X-6, "MCNP6 Users Manual - Code Version 6.1.1 beta,” LA-CP-14-00745, Department of Energy, Los Alamos, June (2014). 


\section{Argonne}

\section{Nuclear Engineering Division}

Argonne National Laboratory

9700 South Cass Avenue, Bldg. 208

Argonne, IL 60439

www.anl.gov 